\title{
Boundary value problems for second order linear difference equations. Application to the computation of the inverse of generalized Jacobi matrices.
}

\author{
A.M. Encinas · M.J. Jiménez
}

Received: date / Accepted: date

\begin{abstract}
We have named generalized Jacobi matrices to those that are practically tridiagonal, except for the two final entries and the two first entries of its first and its last row respectively. This class of matrices encompasses both standard Jacobi and periodic Jacobi matrices that appear in many contexts in pure and applied mathematics. Therefore, the study of the inverse of these matrices becomes of specific interest. However, explicit formulas for inverses are known only in a few cases, in particular when the coefficients of the diagonal entries are subjected to some restrictions.

We will show that the inverse of generalized Jacobi matrices can be raised in terms of the resolution of a boundary value problem associated with a second order linear difference equation. In fact, recent advances in the study of linear difference equations, allow us to compute the solution of this kind of boundary value problems. So, the conditions that ensure the uniqueness of the solution of the boundary value problem leads to the invertibility conditions for the matrix, whereas that solutions for suitable problems provide explicitly the entries of the inverse matrix.
\end{abstract}

Keywords Second order difference equation - Boundary value problem . Generalized Jacobi matrix

\section{Introduction and Preliminaries}

Throughout the paper, $\mathbb{R}$ denotes the field of real numbers. Given a positive integer $n$, we denote by $\mathbb{R}^{n}$ the real coordinate space of $n$ dimensions. Moreover, the components of the vector $\mathrm{v} \in \mathbb{R}^{n}$ are denoted by $v_{j}, j=1, \ldots, n$, i.e., $\mathrm{v}=\left(v_{1}, \ldots, v_{n}\right)^{\top}$.

A.M. Encinas, M.J. Jiménez

Departament de Matemàtiques, Universitat Politècnica de Catalunya, Spain Tel.: +34 934016200

E-mail: andres.marcos.encinas@upc.edu, maria.jose.jimenez@upc.edu 
In addition, we also assume that $0^{0}=1$ and the usual convention that empty sums and empty products are defined as 0 and 1 respectively.

If $n$ is a positive integer, we call generalized Jacobi matrix of order $n+2$ with coefficients $\left\{a_{k}\right\}_{k=0}^{n},\left\{b_{k}\right\}_{k=0}^{n},\left\{c_{k}\right\}_{k=0}^{n} \subset \mathbb{R}, \sigma_{i j} \in \mathbb{R}, i=1,2, j=1,2,3,4$, to the real square matrix $\mathrm{M} \in \mathcal{M}_{n+2}(\mathbb{R})$ of order $n+2$ with the following structure:

$$
\mathrm{M}=\left[\begin{array}{ccccccc}
\sigma_{11} & \sigma_{12} & 0 & \cdots & 0 & \sigma_{13} & \sigma_{14} \\
-c_{0} & b_{1} & -a_{1} & \cdots & 0 & 0 & 0 \\
0 & -c_{1} & b_{2} & \cdots & 0 & 0 & 0 \\
\vdots & \vdots & \ddots & \ddots & \ddots & \vdots & \vdots \\
0 & 0 & 0 & \cdots & b_{n-1} & -a_{n-1} & 0 \\
0 & 0 & 0 & \cdots & -c_{n-1} & b_{n} & -a_{n} \\
\sigma_{21} & \sigma_{22} & 0 & \cdots & 0 & \sigma_{23} & \sigma_{24}
\end{array}\right]
$$

Observe that these matrices are practically tridiagonal, except for the final and first entries of the first and the last row respectively, and include the Jacobi matrices when $\sigma_{13}=\sigma_{14}=\sigma_{21}=\sigma_{22}=0$, and the periodic Jacobi matrices if $\sigma_{13}=\sigma_{22}=0$ and $\sigma_{14} \cdot \sigma_{21} \neq 0$.

As in [22], we have chosen to write down the off-diagonal coefficients with negative sign. This is only a convention, motivated by the relationship between Jacobi matrices and Schrödinger operators on a path, that we will use to analyse the invertibility of the generalized Jacobi matrix.

Notice that the coefficients $a_{0}, b_{0}$ and $c_{n}$ have no influence in the matrix $\mathrm{M}$ and hence they can be chosen arbitrarily. In addition, we also choose arbitrarily the value $b_{n+1}$ and define $a_{n+1}=c_{n}$ and $c_{n+1}=a_{n}$. In Section 2 we justify this last choice. On the other hand, we must make also some assumptions about the coefficients of the matrix $M$ to avoid trivial situations or problems reducible to others with a minor order. First of all, we always assume

$$
\operatorname{rank}\left[\begin{array}{llll}
\sigma_{11} & \sigma_{12} & \sigma_{13} & \sigma_{14} \\
\sigma_{21} & \sigma_{22} & \sigma_{23} & \sigma_{24}
\end{array}\right]=2
$$

otherwise $\mathrm{M}$ is singular. We will also require $a_{k} \neq 0, k=1, \ldots, n$ and $c_{k} \neq 0$, $k=0, \ldots, n-1$, since in other case $\mathrm{M}$ is a reducible matrix and hence the inversion problem leads to the invertibility of a matrix of lower order. Consistently with this assumption, we also suppose that $a_{0}$ and $c_{n}$, and even $a_{n+1}$ and $c_{n+1}$, are also non null.

The matrix $M$ is invertible if and only if for each $f \in \mathbb{R}^{n+2}$ there exists $u \in \mathbb{R}^{n+2}$ such that $M u=f$, so $u$ is the unique solution of the system $M u=f$ if and only if satisfies that

$$
\left\{\begin{array}{l}
\sigma_{11} u_{0}+\sigma_{12} u_{1}+\sigma_{13} u_{n}+\sigma_{14} u_{n+1}=f_{0}, \\
\sigma_{21} u_{0}+\sigma_{22} u_{1}+\sigma_{23} u_{n}+\sigma_{24} u_{n+1}=f_{n+1},
\end{array}\right.
$$

and moreover

$$
-a_{k} u_{k+1}+b_{k} u_{k}-c_{k-1} u_{k-1}=f_{k}, \quad k=1, \ldots, n .
$$

Let us show that we can recognize in the previous identities the structure of a boundary value problem associated with a second order linear difference equation 
or, equivalently, with a Schrödinger operator on a path. This relation provides us the main guide to develop a methodology to determine when $M$ is nonsingular and then, to compute its inverse. Therefore, Chapters 7 and 11 of [7] will be our main reference.

Specifically, we consider the path whose vertices are $\mathbf{I}=\{0, \ldots, n+1\}$ and define its interior as $\stackrel{\circ}{\mathbf{I}}=\{1, \ldots, n\}$ and its boundary as $\delta(\mathbf{I})=\{0, n+1\}$. In addition, given $\boldsymbol{J} \subseteq \mathbf{I}$, we denote by $\mathcal{C}(\boldsymbol{J})$ the vector space of real functions defined on $\mathbf{I}$ and vanishing on $\mathbf{I} \backslash \boldsymbol{J}$ and by $\mathcal{C}^{*}(\boldsymbol{J})$ the set of functions in $\mathcal{C}(\boldsymbol{J})$ that are non null at each vertex of $\boldsymbol{J}$. Therefore, we can identify $\mathcal{C}(\mathbf{I})$ with $\mathbb{R}^{n+2}$ assigning to any $\checkmark \in \mathbb{R}^{n+2}$ the function $v \in \mathcal{C}(\mathbf{I})$ defined as $v(k)=v_{k}$. In particular, we denote by $\varepsilon_{k} \in \mathcal{C}(\mathbf{I})$ the function identified with the $k$-th vector of the standard basis in $\mathbb{R}^{n+2}$, that is, $\varepsilon_{k}(k)=1$ and $\varepsilon_{k}(j)=0$, otherwise. In addition, the three sequences of coefficients $\left\{a_{k}\right\}_{k=0}^{n+1},\left\{b_{k}\right\}_{k=0}^{n+1},\left\{c_{k}\right\}_{k=0}^{n+1}$ are identified with $a, c \in \mathcal{C}^{*}(\mathbf{I})$ and $b \in \mathcal{C}(\mathbf{I})$.

We call conductance on the path $\mathbf{I}$ any function $\gamma: \mathbf{I} \times \mathbf{I} \longrightarrow \mathbb{R}$ such that $\gamma(k, s) \neq 0$ if and only if $|k-s|=1$ and denote by $\Gamma(\mathbf{I})$ the set of conductances on I.

Given $\gamma \in \Gamma(\mathbf{I})$ and $q \in \mathcal{C}(\mathbf{I})$, the Schrödinger operator with potential $q$ and conductivity $\gamma$ is the linear operator $\mathcal{L}_{q}^{\gamma}: \mathcal{C}(\mathbf{I}) \longrightarrow \mathcal{C}(\mathbf{I})$ defined as

$$
\mathcal{L}_{q}^{\gamma}(u)(k)=\sum_{s \in \mathbf{I}} \gamma(k, s)(u(k)-u(s))+q(k) u(k), \quad k \in \mathbf{I} .
$$

Given $f \in \mathcal{C}(\mathbf{I})$ we called Schrödinger equation on $\mathbf{I}$ with data $f$, to the problem of finding $u \in \mathcal{C}(\mathbf{I})$ satisfying

$$
\mathcal{L}_{q}^{\gamma}(u)=f \quad \text { on } \quad \stackrel{\circ}{\mathbf{I}} .
$$

Notice that the values of $f$ at $\delta(\mathbf{I})$ do not have any relevance in the above equation and hence we could have supposed that $f \in \mathcal{C}(\stackrel{\mathbf{I}}{)}$. When $f=0$, then (4) is called homogeneous Schrödinger equation.

If we consider the conductance $\gamma \in \Gamma(\mathbf{I})$ and the potential $q \in \mathcal{C}(\mathbf{I})$ defined as

$$
\begin{aligned}
\gamma(k, k+1)=a_{k}, \quad \gamma(k+1, k) & =c_{k}, & k & =0, \ldots, n, \\
q(k) & =b_{k}-a_{k}-c_{k-1}, & & k=1, \ldots, n+1,
\end{aligned}
$$

and by 0 otherwise, then (3) coincide with the Schrödinger equation (4). Conversely, it is clear that any Schrödinger equation on I corresponds to a set of equations as (3) for the coefficients $a_{k}=\gamma(k, k+1), b_{k}=q(k)+\gamma(k, k+1)+\gamma(k, k-1)$ and $c_{k-1}=\gamma(k, k-1), k=1, \ldots, n$.

Finally, given $\sigma_{1}, \sigma_{2}, \sigma_{3}$ and $\sigma_{4} \in \mathbb{R}$ not simultaneously null, we define boundary linear form or linear boundary condition to the linear map $\mathfrak{c}: \mathcal{C}(\mathbf{I}) \longrightarrow \mathbb{R}$ determined by the expression

$$
\mathfrak{c}(u)=\sigma_{1} u(0)+\sigma_{2} u(1)+\sigma_{3} u(n)+\sigma_{4} u(n+1), \text { for each } u \in \mathcal{C}(\mathbf{I}) .
$$

Then, we can consider $\mathfrak{c}_{1}, \mathfrak{c}_{2}$ as the boundary conditions with coefficients $\sigma_{11}, \sigma_{12}$, $\sigma_{13}, \sigma_{14}$ and $\sigma_{21}, \sigma_{22}, \sigma_{23}, \sigma_{24}$, respectively, and we denote with $\left(\mathfrak{c}_{1}, \mathfrak{c}_{2}\right)$ the pair of boundary conditions determined by the matrix $\mathrm{C}=\left[\begin{array}{llll}\sigma_{11} & \sigma_{12} & \sigma_{13} & \sigma_{14} \\ \sigma_{21} & \sigma_{22} & \sigma_{23} & \sigma_{24}\end{array}\right] \in \mathcal{M}_{2 \times 4}(\mathbb{R})$. 
With this all functional notation, (2) and (3) are equivalent to the boundary value problem on the path $\mathbf{I}$

$$
\mathcal{L}_{q}^{\gamma}(u)=f \quad \text { on } \stackrel{\circ}{\mathbf{I}}, \quad \mathfrak{c}_{1}(u)=f(0) \quad \text { and } \quad \mathfrak{c}_{2}(u)=f(n+1)
$$

In terms of the boundary value problem, the invertibility conditions for $\mathrm{M}$ are exactly the same conditions to ensure that the above boundary value problem is regular, that is, it has a unique solution for each given data. Therefore, the computation of the inverse of $\mathrm{M}$ can be reduced to the calculus of the solution of boundary value problems for suitable data. Implicitly or explicitly, this is the strategy for approaching this kind of problems, see for instance $[5,12,14-16,19,21$, $24,25]$, specially when the matrices are strictly tridiagonal. In that case, the corresponding boundary value problem is a Sturm-Liouville problem and, consequently, in almost all the works to achieve the inversion of tridiagonal matrices is reduced to determine the solutions for initial and final value problems, i.e. [24] and [14,15]. Perhaps the most significant exception is R.K. Mallik's work, see [19], where the boundary vale problem is addressed directly resulting an excessively cumbersome result. In any case, there are no works analyzing the general case of matrices not strictly tridiagonal as $\mathrm{M}$, and in specific problems, the explicit expressions of these solutions are not obtained either.

Therefore, the aim of this work is to determine the solution of the boundary value problem (5) for all kind of boundary conditions, which will allow us to provide the invertibility conditions for $M$ and the explicit expression of its inverse. With this purpose in mind, in Section 2 we provide the main facts about the difference equation related to the boundary value problem. Section 3 is devoted to classifying the boundary value problems in a similar way to that of the second order differential equations. In Section 4 we achieve the effective resolution of (5) in terms of functional kernels that we later determine in Section 5. Next, in Section 6 , the obtained solution is simplified using the previous classification. Finally, in Section 7 we apply the results obtained in previous sections to determine the inverse of generalized Jacobi matrices.

We remark that our methodology is based on solving initial and final value problems and we have proved that it works for general boundary conditions. Since our technique is strongly related to the classification of the boundary conditions, it should must also work in the differential case. The following step in our analysis would be the study of penta-diagonal matrices with perturbations in the corners, which corresponds to fourth-order linear difference equations. This case is by far much more difficult that the second order one, and only specific examples have been discussed in the literature, see for instance $[13,17,18,23]$. In these works, a general formula for the inverse matrix is not provided, but the linear system is treated numerically. For the analytical treatment of this new problem, two main difficulties appear for both fourth-order difference and differential equations. The first one is that it is not enough to solve an initial and a final value problem as in the second order case. The second one is that the boundary conditions for this class of equations are more complex. In fact, there is no systematic classification of these boundary conditions. 


\section{Schrödinger Equations}

Since the solution of a boundary value problem as the one raised in the above section is, in particular, a solution of the corresponding Schrödinger equation (4), that is, the second order difference equation (3), we survey in this section the main facts about these kind of problems. Of course, we assume here that the coefficients $b \in \mathcal{C}(\mathbf{I})$ and $a, c \in \mathcal{C}^{*}(\mathbf{I})$ satisfying $a(n+1)=c(n)$ and $c(n+1)=a(n)$, and hence the associated conductance $\gamma \in \Gamma(\mathbf{I})$ and potential $q \in \mathcal{C}(\mathbf{I})$, have been fixed. Although most of the following results are known, we treat of write them in the framework of this paper and refer the reader to [9] for the proofs of the main results.

Every initial value problem for the Schrödinger equation has a unique solution. Specifically, given $f \in \mathcal{C}(\mathbf{I})$ and $m=0, \ldots, n$, for any $\alpha, \beta \in \mathbb{R}$ there exists a unique $u \in \mathcal{C}(\mathrm{I})$ satisfying

$$
\mathcal{L}_{q}^{\gamma}(u)=f \quad \text { on } \stackrel{\circ}{\mathbf{I}} \text { and } u(m)=\alpha, u(m+1)=\beta .
$$

In particular, when $m=n$, the above problem is also known as final value problem.

If $\mathcal{S}$ denotes the set of solutions of the homogeneous Schrödinger equation, that is, $\mathcal{L}_{q}(u)=0$ on $\stackrel{\circ}{\mathbf{I}}$, then $\mathcal{S}$ is a vector space such that $\operatorname{dim} \mathcal{S}=2$. While for any $f \in \mathcal{C}(\mathbf{I})$, the set $\mathcal{S}(f)$ of solutions of the Schrödinger equation on $\stackrel{\circ}{\mathbf{I}}$ with data $f$ satisfies $\mathcal{S}(f) \neq \emptyset$ and given $u \in \mathcal{S}(f)$, it is satisfied $\mathcal{S}(f)=u+\mathcal{S}$. This property is known as superposition principle.

The Wronskian or Casoratian is the skew-symmetric bilinear form $w[u, v] \in \mathcal{C}(\mathbf{I})$ defined as

$$
w[u, v](k)=\operatorname{det}\left[\begin{array}{cc}
u(k) & v(k) \\
u(k+1) & v(k+1)
\end{array}\right]=u(k) v(k+1)-v(k) u(k+1), \quad 0 \leq k \leq n,
$$

and as $w[u, v](n+1)=w[u, v](n)$, see [1]. Therefore, given $u, v \in \mathcal{C}(\mathbf{I})$, their Wronskian or Casoratian, is $w[u, v]$.

The main Wronskian property permits to identify the basis of the homogeneous Schrödinger equation. To do this, it will be very useful to introduce the companion function defined as

$$
\rho_{\gamma}(k)=\prod_{s=0}^{k-1} \frac{a(s)}{c(s)}, \quad k \in \mathbf{I} .
$$

Notice that $\rho(0)=1$ and moreover $\rho(k) a(k)=\rho(k+1) c(k), k=0, \ldots, n$.

Proposition 1 Given $u, v \in \mathcal{S}$, then

$$
a(k) w[u, v](k)=c(k-1) w[u, v](k-1) \quad \text { for any } k \in \stackrel{\circ}{\mathbf{I}}
$$

Therefore, the multiplication of functions $\rho a w[u, v]$ is constant on $\mathbf{I}$ and moreover it is zero if and only if $u$ and $v$ are linearly dependent.

As an easy by-product of the above result, given $u, v \in \mathcal{S}$, either $w[u, v]=0$ or $w[u, v] \neq 0$ for any $k \in \mathbf{I}$. Moreover, $u$ and $v$ are linearly independent if and only if their Wronskian is non null and then $\{u, v\}$ forms a basis of $\mathcal{S}$. 
The Green function of the Schrödinger equation is $g \in \mathcal{C}(\mathbf{I} \times \mathbf{I})$, defined for any $s \in \mathbf{I}$ as $g(\cdot, s)$, the unique solution of the initial value problem

$$
\begin{aligned}
& \mathcal{L}_{q}^{\gamma}(u)=0 \quad \text { on } \stackrel{\circ}{\mathbf{I}} \text { and } u(s)=0, \quad u(s+1)=-\frac{1}{a(s)}, \quad 0 \leq s \leq n, \\
& \mathcal{L}_{q}^{\gamma}(u)=0 \quad \text { on } \stackrel{\circ}{\mathbf{I}} \text { and } u(n)=\frac{1}{a(n+1)}, \quad u(n+1)=0, \quad s=n+1 .
\end{aligned}
$$

Therefore, $g(s, s)=0$ for any $s \in \mathbf{I}$, whereas $g(s+1, s)=-a(s)^{-1}$ if $s=0, \ldots, n$ and $g(n, n+1)=a(n+1)^{-1}$. Notice that necessarily $g(s, s+1)=c(s)^{-1}$ for any $s=0, \ldots, n$ and, moreover, $g(n+1, n)=-c(n+1)^{-1}$. This relations justifies the choice $a(n+1)=c(n)$ and $c(n+1)=a(n)$ for the coefficients.

Since $u=g(\cdot, k) \in \mathcal{S}$ and $v=g(\cdot, m) \in \mathcal{S}$ for $k, m \in \mathbf{I}$ and moreover if $k \neq n+1$

$$
w[u, v](k)=g(k, k) g(k+1, m)-g(k+1, k) g(k, m)=a(k)^{-1} g(k, m),
$$

we have that $\{u, v\}$ is a basis of $\mathcal{S}$ if and only if $g(k, m) \neq 0$. In particular, $\{g(\cdot, s), g(\cdot, s+1)\}$ is a basis of $\mathcal{S}$ for any $s=0, \ldots, n$. Conversely, if $\{u, v\}$ is a basis of $\mathcal{S}$, then

$$
g(k, s)=\frac{1}{a(s) w[u, v](s)}[v(s) u(k)-u(s) v(k)], \quad k, s \in \mathbf{I} .
$$

or equivalently

$$
g(k, s)=\frac{\rho_{\gamma}(s)}{a(0) w[u, v](0)}[v(s) u(k)-u(s) v(k)], \quad k, s \in \mathbf{I},
$$

where we have taken into account that $a(s) \rho_{\gamma}(s) w[u, v](s)=a(0) w[u, v](0)$ for any $s \in \mathbf{I}$.

The Green function is the main tool for the resolution of any initial value problem, as can be consulted in detail in [9], since for any data provides a solution of the corresponding Schrödinger equation. Specifically, for any $f \in \mathcal{C}(\mathbf{I})$ and any $m=0, \ldots, n$, the function $u \in \mathcal{C}(\mathbf{I})$ given by

$$
u(k)=\sum_{s=\min \{k, m\}+1}^{\max \{k, m\}} g(k, s) f(s), \quad k \in \mathbf{I}
$$

is the unique solution of the initial value problem

$$
\mathcal{L}_{q}^{\gamma}(u)=f \quad \text { on } \stackrel{\circ}{\mathbf{I}} \text { and } u(m)=u(m+1)=0,
$$

see [9, Theorem 4.3].

We end this section by pointing out that to solve the Schrödinger equation for any data it suffices to know $\{u, v\}$ a basis of the corresponding homogeneous equation, since from it we can determine the Green function and hence all the solutions by applying the Superposition Principle.

On the other hand, to obtain a basis of $\mathcal{S}$ it suffices to solve suitable initial value problems; for instance those determined by $u(m)=0, u(m+1)=1$ and $v(m)=1, v(m+1)=0$, for some $m=0, \ldots, n$. From these conditions we can inductively determine $u$ and $v$. However, to find explicit expressions for such a functions is in general a very difficult task, since the obtained formulas, if they exist, are of combinatorial nature and hence excessively cumbersome. We believe that the clearest and unified expressions in this framework are those obtained by the authors in [9]. 


\section{Classification of the boundary value problems}

In this section we will classify the boundary value problems according mainly to what elements of $\delta(\mathbf{I})=\{0, n+1\}$ are involved in the expression of the boundary conditions. Hence, this classification does not depend on the coefficients of the difference operator considered and, therefore, it is more a classification of the own boundary conditions. From this point of view, there exist few distinctions with the treatment of boundary conditions for second order differential equations. So, we follow the structure of the study of this kind of problems given in [7], for us the most complete analysis available in the literature. Following these guidelines, we obtain here new results that could also be applied to the analysis of general boundary value problems for second order differential equations.

Maybe, the main distinction between the discrete and the differential case consists in that in the first one we must to take into account the value of the functions at nodes $k=1$ and $k=n$, since the discrete version of the derivative at the boundary of an interval is $u(0)-u(1)$ on the left and $u(n+1)-u(n)$ on the right. For instance, any general boundary value condition involving the values of $u \in \mathcal{C}(\mathbf{I})$ and its discrete derivative at 0 , must be expressed as

$$
\alpha u(0)+\beta(u(0)-u(1))=\sigma_{1} u(0)+\sigma_{2} u(1), \text { where } \sigma_{1}=\alpha+\beta \text { and } \sigma_{2}=-\beta .
$$

As before, we consider fixed the functions $b \in \mathcal{C}(\mathbf{I}), a, c \in \mathcal{C}^{*}(\mathbf{I})$ such that $a(n+1)=c(n)$ and $c(n+1)=a(n)$, the conductance $\gamma \in \Gamma(\mathbf{I})$ and the potential $q \in \mathcal{C}(\mathbf{I})$ determined by them and hence the corresponding Schrödinger operator $\mathcal{L}_{q}^{\gamma}$

In addition, we also consider the pair of boundary conditions $\left(\mathfrak{c}_{1}, \mathfrak{c}_{2}\right)$ determined by the matrix $\mathrm{C}=\left[\begin{array}{llll}\sigma_{11} & \sigma_{12} & \sigma_{13} & \sigma_{14} \\ \sigma_{21} & \sigma_{22} & \sigma_{23} & \sigma_{24}\end{array}\right]$, where we assume that both conditions are linearly independent, that is, $\operatorname{rank} \mathrm{C}=2$.

With all these coefficients the boundary value problem on the path $\mathbf{I}$, denoted by $\left(\mathcal{L}_{q}^{\gamma}, \mathfrak{c}_{1}, \mathfrak{c}_{2}\right)$, consists in for any data $f \in \mathcal{C}(\mathbf{I})$ find $u \in \mathcal{C}(\mathbf{I})$, called solution, satisfying

$$
\mathcal{L}_{q}^{\gamma}(u)=f \quad \text { on } \stackrel{\circ}{\mathbf{I}}, \quad \mathfrak{c}_{1}(u)=f(0) \text { and } \quad \mathfrak{c}_{2}(u)=f(n+1) .
$$

The boundary value problem is called semi-homogeneous when $f \in \mathcal{C}(\stackrel{\mathbf{I}}{)}$, that is, when it is satisfied $f(0)=f(n+1)=0$, and homogeneous when $f=0$. Clearly the homogeneous boundary value problem always has the null function as a solution. In what follows, we refer this solution as the trivial solution.

The following result shows that we can restrict our study to semi-homogeneous boundary value problems. The key fact is the linear independence of the boundary conditions pair and consequently $\operatorname{rank} C=2$, because it implies that given any values $f(0)$ and $f(n+1)$, we can always find $v \in \mathbb{R}^{4}$ such that $\mathrm{Cv}=(f(0), f(n+1))^{\top}$. Notice that this property is equivalent to be surjective the linear map $\mathfrak{h}: \mathcal{C}(\mathbf{I}) \longrightarrow$ $\mathbb{R}^{2}$ defined as $\mathfrak{h}(u)=\left(\mathfrak{c}_{1}(u), \mathfrak{c}_{2}(u)\right)^{\top}$.

Lemma 1 Let us consider the boundary value problem $\left(\mathcal{L}_{q}^{\gamma}, \mathfrak{c}_{1}, \mathfrak{c}_{2}\right)$ and $f \in \mathcal{C}(\mathbf{I})$. If $v_{1}, v_{2}, v_{3}, v_{4} \in \mathbb{R}$ are such that

$\sigma_{11} v_{1}+\sigma_{12} v_{2}+\sigma_{13} v_{3}+\sigma_{14} v_{4}=f(0) \quad$ and $\quad \sigma_{21} v_{1}+\sigma_{22} v_{2}+\sigma_{23} v_{3}+\sigma_{24} v_{4}=f(n+1)$ 
and we define $g \in \mathcal{C}(\mathbf{I})$ as

$$
\begin{aligned}
g & =f-f(0) \varepsilon_{0}+\left[\left(v_{1}-v_{2}\right) c(0)-v_{2}(a(1)+q(1))\right] \varepsilon_{1}+v_{2} c(1) \varepsilon_{2} \\
& +v_{3} a(n-1) \varepsilon_{n-1}+\left[\left(v_{4}-v_{3}\right) a(n)-v_{3}(c(n-1)+q(n))\right] \varepsilon_{n}-f(n+1) \varepsilon_{n+1},
\end{aligned}
$$

then $g(0)=g(n+1)=0$ and $u \in \mathcal{C}(\mathbf{I})$ is a solution of the boundary value problem with data $f$ if and only if the function

$$
v=u-v_{1} \varepsilon_{0}-v_{2} \varepsilon_{1}-v_{3} \varepsilon_{n}-v_{4} \varepsilon_{n+1}
$$

is a solution of the semi-homogeneous boundary value problem with data $g$.

The fact that the analysis of each boundary value problem is reduced to a semi-homogenoeus one, motivates the following definition.

Definition 1 If $\left(\mathfrak{c}_{1}, \mathfrak{c}_{2}\right)$ and $\left(\hat{\mathfrak{c}}_{1}, \hat{\mathfrak{c}}_{2}\right)$ are two pairs of linearly independent boundary conditions, we called them equivalent if $\operatorname{ker} \mathfrak{c}_{1} \cap \operatorname{ker} \mathfrak{c}_{2}=\operatorname{ker} \hat{\mathfrak{c}}_{1} \cap \operatorname{ker} \hat{\mathfrak{c}}_{2}$. In these circumstances, the boundary value problems $\left(\mathcal{L}_{q}^{\gamma}, \mathfrak{c}_{1}, \mathfrak{c}_{2}\right)$ and $\left(\mathcal{L}_{q}^{\gamma}, \hat{\mathfrak{c}}_{1}, \hat{\mathfrak{c}}_{2}\right)$ are also called equivalent boundary value problems.

The following result characterizes when two pairs of linearly independent boundary conditions are equivalent.

Lemma 2 If $\left(\mathfrak{c}_{1}, \mathfrak{c}_{2}\right)$ and $\left(\hat{\mathfrak{c}}_{1}, \hat{\mathfrak{c}}_{2}\right)$ are the pairs of linearly independent boundary conditions determined by the matrices $\mathrm{C}, \widehat{\mathrm{C}} \in \mathcal{M}_{2 \times 4}(\mathbb{R})$, then these pairs are equivalent if and only if it exists a nonsingular matrix $\mathrm{N} \in \mathcal{M}_{2}(\mathbb{R})$ such that $\widehat{\mathrm{C}}=\mathrm{NC}$.

Proof Let us consider $\mathfrak{h}, \hat{\mathfrak{h}}: \mathcal{C}(\mathbf{I}) \longrightarrow \mathbb{R}^{2}$ the functions defined as $\mathfrak{h}(u)=\left(\mathfrak{c}_{1}(u), \mathfrak{c}_{2}(u)\right)^{\top}$ and $\hat{\mathfrak{h}}(u)=\left(\hat{\mathfrak{c}}_{1}(u), \hat{\mathfrak{c}}_{2}(u)\right)^{\top}$. Clearly the pairs $\left(\mathfrak{c}_{1}, \mathfrak{c}_{2}\right)$ and $\left(\hat{\mathfrak{c}}_{1}, \hat{\mathfrak{c}}_{2}\right)$ are equivalent if and only if ker $\mathfrak{h}=\operatorname{ker} \hat{\mathfrak{h}}$. Moreover, given $N \in \mathcal{M}_{2}(\mathbb{R})$, then $\widehat{C}=N C$ if and only if $\hat{\mathfrak{h}}(u)=\mathrm{Nh}(u)$, for each $u \in \mathcal{C}(\mathbf{I})$. Therefore, if the previous identity is satisfied and $\mathrm{N}$ is invertible, then $\operatorname{ker} \mathfrak{h}=\operatorname{ker} \hat{\mathfrak{h}}$ and hence, the pairs are equivalent.

Conversely, assume that $\operatorname{ker} \mathfrak{h}=\operatorname{ker} \hat{\mathfrak{h}}$. Since $\mathfrak{h}$ and $\hat{\mathfrak{h}}$ are surjective we can consider $u, v, w, y \in \mathcal{C}(\mathbf{I})$ such that

$$
[\mathfrak{h}(u), \mathfrak{h}(v)]=[\hat{\mathfrak{h}}(w), \hat{\mathfrak{h}}(y)]=\left[\begin{array}{ll}
1 & 0 \\
0 & 1
\end{array}\right] .
$$

Therefore, $u$ and $v$ are linearly independent and $w$ and $y$ are also linearly independent. In addition, if $\hat{\mathfrak{h}}(u)=(a, b)^{\top}$ and $\hat{\mathfrak{h}}(v)=(c, d)^{\top}$, then there are $z_{1}, z_{2} \in$ ker $\hat{\mathfrak{h}}$ such that $u=a w+b y+z_{1}$ and $v=c w+d y+z_{2}$.

Let us consider $\mathrm{N}=\left[\begin{array}{ll}a & c \\ b & d\end{array}\right]$ and suppose that there are $\lambda, \mu \in \mathbb{R}$ such that $\mathrm{N}\left[\begin{array}{l}\lambda \\ \mu\end{array}\right]=\left[\begin{array}{l}0 \\ 0\end{array}\right]$. Then, $x=\lambda u+\mu v \in \operatorname{ker} \hat{\mathfrak{h}}=\operatorname{ker} \mathfrak{h}$ and hence, $\mathfrak{h}(x)=(\lambda, \mu)^{\top}=(0,0)$, that is, $\lambda=\mu=0$. Therefore, the matrix $\mathrm{N}$ is invertible.

If $x \in \mathcal{C}(\mathbf{I})$ and $\mathfrak{h}(x)=(\alpha, \beta)^{\top}$, then it exists $\hat{z} \in \operatorname{ker} \mathfrak{h}=\operatorname{ker} \hat{\mathfrak{h}}$ such that

$$
x=\alpha u+\beta v+\hat{z}=\alpha(a w+b y)+\beta(c w+d y)+\alpha z_{1}+\beta z_{2}+\hat{z},
$$


which implies $\hat{\mathfrak{h}}(x)=\mathbf{N}\left[\begin{array}{l}\alpha \\ \beta\end{array}\right]=\mathbf{N h}(x)$, since $\alpha z_{1}+\beta z_{2}+\hat{z} \in \operatorname{ker} \hat{\mathfrak{h}}$.

Given $\left(\mathfrak{c}_{1}, \mathfrak{c}_{2}\right)$ the pair of linear independent boundary conditions determined

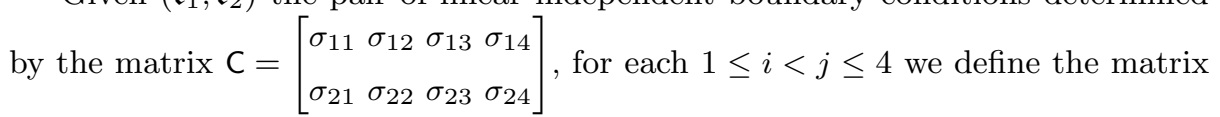
$\mathrm{C}_{i j}=\left[\begin{array}{cc}\sigma_{1 i} & \sigma_{1 j} \\ \sigma_{2 i} & \sigma_{2 j}\end{array}\right]$ and the value $d_{i j}=\operatorname{det} \mathrm{C}_{i j}$. So, $\mathrm{C}=\left[\mathrm{C}_{12} \mathrm{C}_{34}\right]$ and as $\operatorname{rank} \mathrm{C}=2$, then $\sum_{1 \leq i<j \leq 4}\left|d_{i j}\right|>0$.

Observe that if $\left(\hat{\mathfrak{c}}_{1}, \hat{\mathfrak{c}}_{2}\right)$ is the pair of linearly independent boundary conditions determined by $\widehat{C} \in \mathcal{M}_{2 \times 4}(\mathbb{R})$ then from Lemma $2, \widehat{C}$ is equivalent to $\mathrm{C}$ if and only if it exists a nonsingular matrix $\mathrm{N} \in \mathcal{M}_{2}(\mathbb{R})$ such that $\widehat{\mathrm{C}}=\left[\mathrm{NC}_{12} \mathrm{NC}_{34}\right]$.

We are now ready for the classification of our boundary value problems. Since the boundary conditions involve the values of the functions in the nodes $0,1, n$ and $n+1$, the classification will attend precisely to which ones are taken into account.

Definition 2 The pair of boundary conditions $\left(\mathfrak{c}_{1}, \mathfrak{c}_{2}\right)$ is unilateral, or the conditions $\mathfrak{c}_{1}$ and $\mathfrak{c}_{2}$ are unilateral, if either $C_{34}=0$, in which case we call them initial; or $\mathrm{C}_{12}=0$, in which case we call them final.

Lemma 3 Let us consider $\left(\mathfrak{c}_{1}, \mathfrak{c}_{2}\right)$ a pair of unilateral conditions. Then, any equivalent pair is also unilateral. In addition, the following properties hold:

(i) If the pair is initial, then $d_{12} \neq 0$ and it is equivalent to the pair $\left(\hat{\mathfrak{c}}_{1}, \hat{\mathfrak{c}}_{2}\right)$, where $\hat{\mathfrak{c}}_{1}(u)=u(0)$ and $\hat{\mathfrak{c}}_{2}(u)=u(1)$.

(ii) If the pair is final, then $d_{34} \neq 0$ and it is equivalent to the pair $\left(\hat{\mathfrak{c}}_{1}, \hat{\mathfrak{c}}_{2}\right)$, where $\hat{\mathfrak{c}}_{1}(u)=u(n)$ and $\hat{\mathfrak{c}}_{2}(u)=u(n+1)$.

The above result shows that unilateral boundary conditions are in fact initial or final, that is, they are conditions such the ones defined in Section 2. Therefore, the remain of this section will be devoted to study genuine boundary conditions, that is, boundary conditions that are not unilateral.

Definition 3 Let us consider the pair of boundary conditions $\left(\mathfrak{c}_{1}, \mathfrak{c}_{2}\right)$ satisfying $\mathrm{C}_{12} \neq 0$ and $\mathrm{C}_{34} \neq 0$. The pair is called separable at node 0 if $d_{34}=0$, and separable at node $n+1$ if $d_{12}=0$. The pair is simply separable if it is separable at 0 and at $n+1$ simultaneously, that is, if $d_{12}=d_{34}=0$.

Note that the hypothesis $C_{12}, C_{34} \neq 0$ determines that the pair $\left(\mathfrak{c}_{1}, \mathfrak{c}_{2}\right)$ is not unilateral. Reciprocally, if $\mathrm{C}_{12} \neq 0$ and $d_{12}=0$, necessarily $\mathrm{C}_{34} \neq 0$ and, analogously, if $\mathrm{C}_{34} \neq 0$ and $d_{34}=0$, necessarily $\mathrm{C}_{12} \neq 0$, since rank $\mathrm{C}=2$. On the other hand, if the pair $\left(\mathfrak{c}_{1}, \mathfrak{c}_{2}\right)$ is separable, either at 0 , or at $n+1$, then any equivalent pair has the same property.

Proposition 2 The following properties hold:

(i) If $\left(\mathfrak{c}_{1}, \mathfrak{c}_{2}\right)$ is separable at 0 , then is equivalent to the pair determined by a matrix of the form $\left[\begin{array}{cccc}a_{11} & a_{12} & 0 & 0 \\ a_{21} & a_{22} & a_{23} & a_{24}\end{array}\right]$, where $\left(\left|a_{11}\right|+\left|a_{12}\right|\right)\left(\left|a_{23}\right|+\left|a_{24}\right|\right)>0$ and $a_{21} \cdot a_{22}=0$. 
(ii) If $\left(\mathfrak{c}_{1}, \mathfrak{c}_{2}\right)$ is separable at $n+1$, then is equivalent to the pair determined by a matrix of the form $\left[\begin{array}{cccc}a_{11} & a_{12} & a_{13} & a_{14} \\ 0 & 0 & a_{23} & a_{24}\end{array}\right]$, where $\left(\left|a_{11}\right|+\left|a_{12}\right|\right)\left(\left|a_{23}\right|+\left|a_{24}\right|\right)>0$ and $a_{13} \cdot a_{14}=0$.

(iii) If $\left(\mathfrak{c}_{1}, \mathfrak{c}_{2}\right)$ is separable, then is equivalent to the pair determined by a matrix of the form $\left[\begin{array}{cccc}a_{11} & a_{12} & 0 & 0 \\ 0 & 0 & a_{23} & a_{24}\end{array}\right]$, where $\left(\left|a_{11}\right|+\left|a_{12}\right|\right)\left(\left|a_{23}+\right| a_{24} \mid\right)>0$.

Proof Since the proofs for items (i) and (ii) are identical, we will just show the case (i).

If $\sigma_{23}=\sigma_{24}=0$, then $\left|\sigma_{13}\right|+\left|\sigma_{14}\right|>0$ and, moreover, the linear independence of the boundary conditions imposes $\left|\sigma_{21}\right|+\left|\sigma_{22}\right|>0$. Then, $\left(\mathfrak{c}_{1}, \mathfrak{c}_{2}\right)$ is equivalent to $\left(\mathfrak{c}_{2}, \mathfrak{c}_{1}\right)$, which is determined by the matrix $\left[\begin{array}{cccc}\sigma_{21} & \sigma_{22} & 0 & 0 \\ \sigma_{11} & \sigma_{12} & \sigma_{13} & \sigma_{14}\end{array}\right]$.

If $\left|\sigma_{23}\right|+\left|\sigma_{24}\right|>0$, since $d_{34}=0$, it exists $\alpha \in \mathbb{R}$ such that $\left(\sigma_{13}, \sigma_{14}\right)=$ $\alpha\left(\sigma_{23}, \sigma_{24}\right)$ and if we consider $\mathbf{L}=\left[\begin{array}{cc}1 & -\alpha \\ 0 & 1\end{array}\right]$, then $\mathbf{L C}=\left[\begin{array}{cccc}\hat{\sigma}_{11} & \hat{\sigma}_{12} & 0 & 0 \\ \sigma_{21} & \sigma_{22} & \sigma_{23} & \sigma_{24}\end{array}\right]$ and, moreover, $\left|\hat{\sigma}_{11}\right|+\left|\hat{\sigma}_{12}\right|>0$, since $\operatorname{rankLC}=2$.

Definitely, the pair $\left(\mathfrak{c}_{1}, \mathfrak{c}_{2}\right)$ is equivalent to the pair determined by the matrix

$$
\left[\begin{array}{cccc}
\hat{\sigma}_{11} & \hat{\sigma}_{12} & 0 & 0 \\
\hat{\sigma}_{21} & \hat{\sigma}_{22} & \hat{\sigma}_{23} & \hat{\sigma}_{24}
\end{array}\right], \quad \text { where }\left(\left|\hat{\sigma}_{11}\right|+\left|\hat{\sigma}_{12}\right|\right)\left(\left|\hat{\sigma}_{23}\right|+\left|\hat{\sigma}_{24}\right|\right)>0
$$

On the other hand, if we consider $N=\left[\begin{array}{cc}1 & 0 \\ -\frac{\hat{\sigma}_{21}}{\sigma_{11}} & 1\end{array}\right]$ when $\hat{\sigma}_{11} \neq 0$, while $\mathbf{N}=\left[\begin{array}{cc}1 & 0 \\ -\frac{\hat{\sigma}_{22}}{\sigma_{12}} & 1\end{array}\right]$ when $\hat{\sigma}_{12} \neq 0$, then $\left[\begin{array}{cccc}a_{11} & a_{12} & 0 & 0 \\ a_{21} & a_{22} & a_{23} & a_{24}\end{array}\right]=\mathrm{N}\left[\begin{array}{cccc}\hat{\sigma}_{11} & \hat{\sigma}_{12} & 0 & 0 \\ \hat{\sigma}_{21} & \hat{\sigma}_{22} & \hat{\sigma}_{23} & \hat{c}_{24}\end{array}\right]$ has the required properties.

(iii) Since the pair $\left(\mathfrak{c}_{1}, \mathfrak{c}_{2}\right)$ is separable at 0 , applying (i) it is equivalent to a pair determined by a matrix of the form $\left[\begin{array}{cccc}a_{11} & a_{12} & 0 & 0 \\ a_{21} & a_{22} & a_{23} & a_{24}\end{array}\right]$, where $\left(\left|a_{11}\right|+\left|a_{12}\right|\right)\left(\left|a_{23}\right|+\right.$ $\left.\left|a_{24}\right|\right)>0$ and $a_{21} \cdot a_{22}=0$.

Since the pair $\left(\mathfrak{c}_{1}, \mathfrak{c}_{2}\right)$ is also separable at $n+1$, necessarily $a_{11} a_{22}=a_{12} a_{21}$, which implies that it exists $\alpha \in \mathbb{R}$ such that $\left(a_{21}, a_{22}\right)=\alpha\left(a_{11}, a_{12}\right)$. If we consider now the matrix $\mathrm{K}=\left[\begin{array}{cc}1 & 0 \\ -\alpha & 1\end{array}\right]$, then $\mathrm{K}\left[\begin{array}{cccc}a_{11} & a_{12} & 0 & 0 \\ a_{21} & a_{22} & a_{23} & a_{24}\end{array}\right]$ has the required properties.

The boundary conditions that are separable are probably the most popular and known boundary conditions and hence they occupy a prominent place in the analysis of this class of problems.

Definition 4 The separable conditions determined by the matrix

$$
\mathbf{C}=\left[\begin{array}{cccc}
\sigma_{11} & \sigma_{12} & 0 & 0 \\
0 & 0 & \sigma_{23} & \sigma_{24}
\end{array}\right]
$$

where $\left(\left|\sigma_{11}\right|+\left|\sigma_{12}\right|\right)\left(\left|\sigma_{23}\right|+\left|\sigma_{24}\right|\right)>0$ are called Sturm-Liouville conditions. 
Next we will associate to the separable conditions the usual denomination in the literature for boundary conditions.

Definition 5 Let us consider $\mathfrak{c}$ the linear boundary condition with coefficients $\sigma_{1}, \sigma_{2}, \sigma_{3}$ and $\sigma_{4}$, where $\left|\sigma_{1}\right|+\left|\sigma_{2}\right|+\left|\sigma_{3}\right|+\left|\sigma_{4}\right|>0$.

(i) The condition $\mathfrak{c}$ is called separable at 0 if $\sigma_{3}=\sigma_{4}=0$. Moreover, if $\sigma_{1} \cdot \sigma_{2}=0$, then $\mathfrak{c}$ is called Dirichlet condition at 0 , interior if $\sigma_{2} \neq 0$ and exterior if $\sigma_{1} \neq 0$. If $\sigma_{1} \cdot \sigma_{2} \neq 0, \mathfrak{c}$ is called Robin condition at 0 . In particular, when $\sigma_{2}=-\sigma_{1}, \mathfrak{c}$ is called Neumann condition at 0 .

(ii) The condition $\mathfrak{c}$ is separable at $n+1$ if $\sigma_{1}=\sigma_{2}=0$. Moreover, if $\sigma_{3} \cdot \sigma_{4}=0$, then $\mathfrak{c}$ is called Dirichlet condition at $n+1$, interior if $\sigma_{3} \neq 0$ and exterior if $\sigma_{4} \neq 0$. If $\sigma_{3} \cdot \sigma_{4} \neq 0, \mathfrak{c}$ is called Robin condition at $n+1$. In particular, if $\sigma_{3}=-\sigma_{4}, \mathfrak{c}$ is called Neumann condition at $n+1$.

If $\mathfrak{c}$ is a Dirichlet boundary condition at 0 , then it is equivalent to the condition $\hat{\mathfrak{c}}(u)=u(0)$ if it is exterior, or to $\hat{\mathfrak{c}}(u)=u(1)$ if it is interior, and an analogous situation arises for the Dirichlet conditions at $n+1$. On the other hand, if we assume fixed the functions $a, c \in \mathcal{C}^{*}(\mathbf{I})$, if $\mathfrak{c}$ is a Robin boundary condition at 0 , then it is equivalent to the Robin condition $\hat{\mathfrak{c}}(u)=c_{1} u(0)-a(0) u(1)$. In particular, when it is a Neumann boundary condition at 0 , it is equivalent to the Neumann condition $\hat{\mathfrak{c}}(u)=a(0) u(0)-a(0) u(1)$. Analogously, if $\mathfrak{c}$ is a Robin boundary condition at $n+1$, then it is equivalent to the Robin condition $\hat{\mathfrak{c}}(u)=c_{4} u(n+1)-c(n) u(n)$ and, in particular, when it is a Neumann boundary condition at $n+1$, it is equivalent to the Neumann condition $\hat{\mathfrak{c}}(u)=c(n) u(n+1)-c(n) u(n)$.

In what follows, when we refer to Dirichlet conditions, whether they are interior or exterior, we will be assuming that the Dirichlet conditions are the ones we have just defined above. Similarly, when we refer to Robin or Neumann conditions in any of the two nodes of $\delta(\mathbf{I})$, we will also be referring to the Robin or Neumann conditions that we have just described.

In Proposition 2 we have shown that any separable pair of boundary conditions, at 0 or at $n+1$, is equivalent to a pair in which at least one condition is separable, at 0 or at $n+1$, respectively. If the condition is separable at 0 , then either it is equivalent to the Dirichlet condition, or to the Robin condition. Analogously, if the condition is separable at $n+1$, then it is either equivalent to the Dirichlet condition, or to Robin condition.

We will finish the classification of boundary conditions introducing what we call of periodic type. This type of conditions also includes those that, in the continuous case, are treated in [7, Chapter 8.3] and which we named here as periodic of second kind.

Definition 6 The pair $\left(\mathfrak{c}_{1}, \mathfrak{c}_{2}\right)$ is a set of periodic conditions if it is neither unilateral, nor separable at 0 or at $n+1$. Hence, the pair is periodic if it is determined by a matrix

$$
C=\left[\begin{array}{llll}
\sigma_{11} & \sigma_{12} & \sigma_{13} & \sigma_{14} \\
\sigma_{21} & \sigma_{22} & \sigma_{23} & \sigma_{24}
\end{array}\right],
$$

where $d_{12} \cdot d_{34} \neq 0$. The conditions are named periodic of first kind or periodic of second kind if, besides, $d_{23}=0$ or $d_{23} \neq 0$, respectively.

Lemma 4 Let us consider $\left(\mathfrak{c}_{1}, \mathfrak{c}_{2}\right)$ a pair of periodic conditions. 
(i) If the pair is periodic of first kind, then it is equivalent to the pair determined by a matrix of the form $\left[\begin{array}{cccc}a_{11} & a_{12} & a_{13} & a_{14} \\ a_{21} & 0 & 0 & a_{24}\end{array}\right]$, where $a_{12} \cdot a_{13} \cdot a_{21} \cdot a_{24} \neq 0$.

(ii) If the pair is periodic of second kind, then it is equivalent to the pair determined by the matrix $\left[\begin{array}{cccc}a_{11} & -a(0) & 0 & a_{14} \\ a_{21} & 0 & -c(n) & a_{24}\end{array}\right]$, where $a_{21} \cdot a_{14} \neq 0$.

\section{Regular boundary value problems}

Once the boundary value problems on a finite path are classified, our purpose now is to achieve their effective resolution. As in [4], we will focus on regular problems, that is, in those problems for which there is only one solution. Our techniques are directed towards the determination of the so-called resolvent kernels. As we will see, the treatment that we develop to determine such kernels represents an extension of more or less known results in the discrete field and also in the continuous one, but usually exclusively referred to the treatment of Sturm-Liouville conditions. We will show here that the process of determining the resolvent kernel and, therefore, the resolution of the boundary value problems, always depends on an appropriate choice of solutions of the corresponding Schrödinger homogeneous equation. The problem statement here is slightly different and more general than the one followed in [4], where only equations with constant coefficients were analyzed. It should also be highlighted that our techniques are also valid for the differential case and can be used in that context without major modifications.

In this section we consider fixed the functions $b \in \mathcal{C}(\mathbf{I}), a, c \in \mathcal{C}^{*}(\mathbf{I})$ such that $a(n+1)=c(n)$ and $c(n+1)=a(n)$, the conductance $\gamma \in \Gamma(\mathbf{I})$ and the potential $q \in$ $\mathcal{C}(\mathbf{I})$ determined by them and hence the corresponding Schrödinger operator $\mathcal{L}_{q}^{\gamma}$. In addition, we also consider the pair of boundary conditions $\left(\mathfrak{c}_{1}, \mathfrak{c}_{2}\right)$ determined by the matrix $\mathrm{C}=\left(\sigma_{i j}\right) \in \mathcal{M}_{2 \times 4}(\mathbb{R})$, where we assume that rank $\mathrm{C}=2$, and the values $d_{i j}=\sigma_{1 i} \sigma_{2 j}-\sigma_{2 i} \sigma_{1 j}, 1 \leq i<j \leq 4$

According to $(9)$, we consider $\left(\mathcal{L}_{q}^{\gamma}, \mathfrak{c}_{1}, \mathfrak{c}_{2}\right)$, the boundary value problem on the path I

$$
\mathcal{L}_{q}^{\gamma}(u)=f, \quad \text { on } \quad \stackrel{\circ}{\mathbf{I}}, \quad \mathfrak{c}_{1}(u)=f(0) \text { and } \quad \mathfrak{c}_{2}(u)=f(n+1),
$$

and recall that this problem is equivalent to the system (??). Moreover, we must to pay attention on the associated Schrödinger equation $\mathcal{L}_{q}^{\gamma}(u)=f$ on $\stackrel{\circ}{\mathbf{I}}$, since any solution of the boundary value problem is, in particular, a solution of the Schrödinger equation. We always denote by $\mathcal{S}$ the space of solutions of the homogeneous Schrödinger equation.

Lemma 5 If $\left\{z_{1}, z_{2}\right\}$ is a basis of solutions of the homogeneous Schrödinger equation $\mathcal{L}_{q}^{\gamma}(u)=0$ and $y$ is a particular solution of the Schrödinger equation with data $f \in \mathcal{C}(\mathbf{I})$, then $u=\alpha z_{1}+\beta z_{2}+y$ with $\alpha, \beta \in \mathbb{R}$, is a solution of the boundary value problem

$$
\mathcal{L}_{q}(u)=f \quad \text { en } \quad \stackrel{\circ}{\mathbf{I}}, \quad \mathfrak{c}_{1}(u)=f(0) \quad y \quad \mathfrak{c}_{2}(u)=f(n+1),
$$

if and only if $\alpha$ and $\beta$ are solutions of the linear system

$$
\left[\begin{array}{ll}
\mathfrak{c}_{1}\left(z_{1}\right) & \mathfrak{c}_{1}\left(z_{2}\right) \\
\mathfrak{c}_{2}\left(z_{1}\right) & \mathfrak{c}_{2}\left(z_{2}\right)
\end{array}\right]\left[\begin{array}{l}
a \\
b
\end{array}\right]=\left[\begin{array}{c}
f(0)-\mathfrak{c}_{1}(y) \\
f(n+1)-\mathfrak{c}_{2}(y)
\end{array}\right]
$$


In particular, $u=\alpha z_{1}+\beta z_{2}$ is a solution of the homogeneous boundary value problem if and only if $\alpha$ and $\beta$ are solutions of the homogeneous linear system

$$
\left[\begin{array}{ll}
\mathfrak{c}_{1}\left(z_{1}\right) & \mathfrak{c}_{1}\left(z_{2}\right) \\
\mathfrak{c}_{2}\left(z_{1}\right) & \mathfrak{c}_{2}\left(z_{2}\right)
\end{array}\right]\left[\begin{array}{l}
a \\
b
\end{array}\right]=\left[\begin{array}{l}
0 \\
0
\end{array}\right]
$$

Notice that since the particular solution of the Schrödinger equation does not depend on $f(0)$ and on $f(n+1)$, when $f$ goes over $\mathcal{C}(\mathbf{I})$, then the right term of the system

$$
\left[\begin{array}{l}
\mathfrak{c}_{1}\left(z_{1}\right) \mathfrak{c}_{1}\left(z_{2}\right) \\
\mathfrak{c}_{2}\left(z_{1}\right) \mathfrak{c}_{2}\left(z_{2}\right)
\end{array}\right]\left[\begin{array}{l}
a \\
b
\end{array}\right]=\left[\begin{array}{c}
f(0)-\mathfrak{c}_{1}(y) \\
f(n+1)-\mathfrak{c}_{2}(y)
\end{array}\right]
$$

goes over all $\mathbb{R}^{2}$. Therefore, the boundary value problem (9) has a solution for any $f \in \mathcal{C}(\mathbf{I})$ if and only if the homogeneous boundary value problem has the trivial solution as its unique solution. Moreover, in this case the boundary value problem has a unique solution for each data $f \in \mathcal{C}(\mathbf{I})$.

Definition 7 The boundary value problem $\left(\mathcal{L}_{q}^{\gamma}, \mathfrak{c}_{1}, \mathfrak{c}_{2}\right)$ is called regular if the solution of the corresponding homogeneous problem is unique, and so the trivial one.

After Lemma 5 , it is clear that the matrix $\left[\begin{array}{ll}\mathfrak{c}_{1}\left(z_{1}\right) & \mathfrak{c}_{1}\left(z_{2}\right) \\ \mathfrak{c}_{2}\left(z_{1}\right) & \mathfrak{c}_{2}\left(z_{2}\right)\end{array}\right]$ plays a main role in the analysis of boundary value problems and motivates the following concept, that seems to be new not only for the discrete framework. The authors believe that its implementation in the differentiable case would facilitate the study of regular problems in that setting.

Definition 8 We called Wronskian of the boundary conditions pair $\left(\mathfrak{c}_{1}, \mathfrak{c}_{2}\right)$ to the function $W: \mathcal{C}(\mathbf{I}) \times \mathcal{C}(\mathbf{I}) \longrightarrow \mathbb{R}$ defined as

$$
W[u, v]=\operatorname{det}\left[\begin{array}{ll}
\mathfrak{c}_{1}(u) & \mathfrak{c}_{1}(v) \\
\mathfrak{c}_{2}(u) & \mathfrak{c}_{2}(v)
\end{array}\right]=\mathfrak{c}_{1}(u) \mathfrak{c}_{2}(v)-\mathfrak{c}_{1}(v) \mathfrak{c}_{2}(u), \quad u, v \in \mathcal{C}(\mathbf{I}) .
$$

Clearly, $W$ is a skew-symmetric bilinear form and, hence, if $\phi=a_{1} u+b_{1} v$ and $\psi=a_{2} u+b_{2} v$, then

$$
W[\phi, \psi]=W[u, v] \operatorname{det}\left[\begin{array}{ll}
a_{1} & b_{1} \\
a_{2} & b_{2}
\end{array}\right] .
$$

Therefore, if $\mathcal{S}$ is the space of solutions of the homogeneous Schrödinger equation then either $W[u, v]=0$ for any basis $\{u, v\}$ of $\mathcal{S}$ or $W[u, v] \neq 0$ for any basis $\{u, v\}$ of $\mathcal{S}$. Moreover we have a more precise property, as is shown in the following result.

Proposition 3 If $g: \mathbf{I} \times \mathbf{I} \longrightarrow \mathbb{R}$ is the Green function of the Schrödinger equation, then the function $D: \mathbf{I} \longrightarrow \mathbb{R}$ defined as $D(n+1)=D(n)$ and

$$
D(s)=\frac{a(0) a(s)}{\rho_{\gamma}(s+1)} W[g(\cdot, s), g(\cdot, s+1)], \quad s=0, \ldots, n,
$$

is constant and its value is given by

$d_{12}-a(0)\left(d_{13} g(n, 0)+d_{14} g(n+1,0)\right)-c(0)\left(d_{23} g(n, 1)+d_{24} g(n+1,1)\right)+\frac{d_{34} a(0)}{a(n) \rho_{\gamma}(n)}$. 
Proof If we consider $u=g(\cdot, 0)$ y $v=g(\cdot, 1)$, then $w[u, v](0)=a(0)^{-1} c(0)^{-1}$ and moreover, (6) and Proposition 1 establish that

$g(\cdot, s)=\frac{1}{a(s) w[u, v](s)}[v(s) u-u(s) v]=c(0) \rho_{\gamma}(s)[v(s) u-u(s) v], \quad s=0, \ldots, n+1$.

Applying now (10), we obtain

$$
\begin{aligned}
W[g(\cdot, s), g(\cdot, s+1)] & =c(0)^{2} \rho_{\gamma}(s+1) \rho_{\gamma}(s) w[u, v](s) W[u, v] \\
& =a(0) c(0)^{2} \rho_{\gamma}(s+1) a(s)^{-1} w[u, v](0) W[u, v] \\
& =c(0) \rho_{\gamma}(s+1) a(s)^{-1} W[u, v], \quad s=0, \ldots, n,
\end{aligned}
$$

and then, it is satisfied

$$
D(s)=a(0) c(0) W[u, v]=D(0),
$$

since $\rho_{\gamma}(1)=a(0) c(0)^{-1}$. On the other hand,

$$
W[u, v]=\operatorname{det}\left[\begin{array}{ll}
\mathfrak{c}_{1}(u) & \mathfrak{c}_{1}(v) \\
\mathfrak{c}_{2}(u) & \mathfrak{c}_{2}(v)
\end{array}\right]=\operatorname{det}\left[\begin{array}{llll}
\sigma_{11} & \sigma_{12} & \sigma_{13} & \sigma_{14} \\
\sigma_{21} & \sigma_{22} & \sigma_{23} & \sigma_{24}
\end{array}\right]\left[\begin{array}{cc}
0 & c(0)^{-1} \\
-a(0)^{-1} & 0 \\
g(n, 0) & g(n, 1) \\
g(n+1,0) & g(n+1,1)
\end{array}\right]
$$

so the expression of $D(0)$ is obtained applying the Cauchy-Binet Identity and taking into account that from Proposition $1, w[u, v](n)=a(n)^{-1} c(0)^{-1} \rho_{\gamma}(n)^{-1}$.

Definition 9 We call determinant of the boundary value problem $\left(\mathcal{L}_{q}^{\gamma}, \mathfrak{c}_{1}, \mathfrak{c}_{2}\right)$ to the value

$$
D_{\mathfrak{c}_{1}, \mathfrak{c}_{2}}^{a, b, c}=a(0) c(0) a(n) \rho_{\gamma}(n) W[g(\cdot, 0), g(\cdot, 1)],
$$

where $g \in \mathcal{C}(\mathbf{I} \times \mathbf{I})$ is the Green function of the Schrödinger equation.

Notice that from the above Proposition we have that

$$
\begin{aligned}
D_{\mathfrak{c}_{1}, \mathfrak{c}_{2}}^{a, b} & =a(n) \rho_{\gamma}(n) d_{12}+a(0) d_{34}-a(0) a(n) \rho_{\gamma}(n)\left(d_{13} g(n, 0)+d_{14} g(n+1,0)\right) \\
& -c(0) a(n) \rho_{\gamma}(n)\left(d_{23} g(n, 1)+d_{24} g(n+1,1)\right) .
\end{aligned}
$$

The next result summarizes the necessary and sufficient conditions under which the boundary value problem is regular.

Proposition 4 The following assertions are equivalent:

(i) The boundary value problem $\left(\mathcal{L}_{q}^{\gamma}, \mathfrak{c}_{1}, \mathfrak{c}_{2}\right)$ is regular.

(ii) For any $f \in \mathcal{C}(\mathbf{I})$ the corresponding boundary value problem has a unique solution.

(iii) $D_{\mathfrak{c}_{1}, \mathfrak{c}_{2}}^{a, b, c} \neq 0$.

(iv) $W[u, v] \neq 0$ when $\{u, v\}$ is a basis of $\mathcal{S}$.

Corollary 1 If the boundary value problem $\left(\mathcal{L}_{q}^{\gamma}, \mathfrak{c}_{1}, \mathfrak{c}_{2}\right)$ is regular and $u, v \in \mathcal{C}(\mathbf{I})$ are solutions of the homogeneous Schrödinger equation on $\stackrel{\circ}{\mathbf{I}}$, then $u$ and $v$ are linearly independent if and only if $W[u, v] \neq 0$. 
Definition 10 Let us consider $\left(\mathcal{L}_{q}^{\gamma}, \mathfrak{c}_{1}, \mathfrak{c}_{2}\right)$ a regular boundary value problem.

We call resolvent kernel of the boundary value problem to $R_{\mathfrak{c}_{1}, \mathfrak{c}_{2}}^{a, b, c}: \mathbf{I} \times \mathbf{I} \longrightarrow \mathbb{R}$ characterized by satisfying for any $s \in \mathbf{I}$ the identities

$$
\mathcal{L}_{q}^{\gamma}\left(R_{\mathfrak{c}_{1}, \mathfrak{c}_{2}}^{a, b, c}(\cdot, s)\right)=\varepsilon_{s} \text { on } \stackrel{\circ}{\mathbf{I}}, \mathfrak{c}_{1}\left(R_{\mathfrak{c}_{1}, \mathfrak{c}_{2}}^{a, b, c}(\cdot, s)\right)=\varepsilon_{s}(0), \mathfrak{c}_{2}\left(R_{\mathfrak{c}_{1}, \mathfrak{c}_{2}}^{a, b, c}(\cdot, s)\right)=\varepsilon_{s}(n+1) .
$$

We call Green kernel of the boundary value problem to $G_{\mathfrak{c}_{1}, \mathfrak{c}_{2}}^{a, b, c}: \mathbf{I} \times \mathbf{I} \longrightarrow \mathbb{R}$ characterized by satisfying $G_{\mathfrak{c}_{1}, \mathfrak{c}_{2}}^{a, b, c}(\cdot, s)=0$ if $s \in \delta(\mathbf{I})$ and if $s \in \stackrel{\circ}{\mathbf{I}}$ by

$$
\mathcal{L}_{q}^{\gamma}\left(G_{\mathfrak{c}_{1}, \mathfrak{c}_{2}}^{a, b, c}(\cdot, s)\right)=\varepsilon_{s} \quad \text { on } \quad \stackrel{\circ}{\mathbf{I}}, \quad \mathfrak{c}_{1}\left(G_{\mathfrak{c}_{1}, \mathfrak{c}_{2}}^{a, b}(\cdot, s)\right)=\mathfrak{c}_{2}\left(G_{\mathfrak{c}_{1}, \mathfrak{c}_{2}}^{a, b}(\cdot, s)\right)=0 .
$$

We call Poisson kernel of the boundary value problem to $P_{\mathfrak{c}_{1}, \mathfrak{c}_{2}}^{a, b}: \mathbf{I} \times \mathbf{I} \longrightarrow \mathbb{R}$ characterized by $P_{\mathfrak{c}_{1}, \mathfrak{c}_{2}}^{a, b}(\cdot, s)=0$ if $s \in \stackrel{\circ}{\mathbf{I}}$ and if $s \in \delta(\mathbf{I})$ by

$$
\mathcal{L}_{q}^{\gamma}\left(P_{\mathfrak{c}_{1}, \mathfrak{c}_{2}}^{a, b}(\cdot, s)\right)=0 \text { on } \stackrel{\circ}{\mathbf{I}}, \mathfrak{c}_{1}\left(P_{\mathfrak{c}_{1}, \mathfrak{c}_{2}}^{a, b, c}(\cdot, s)\right)=\varepsilon_{s}(0), \mathfrak{c}_{2}\left(P_{\mathfrak{c}_{1}, \mathfrak{c}_{2}}^{a, b}(\cdot, s)\right)=\varepsilon_{s}(n+1) .
$$

Observe that $\left\{P_{\mathfrak{c}_{1}, \mathfrak{c}_{2}}^{a, b, c}(\cdot, 0), P_{\mathfrak{c}_{1}, \mathfrak{c}_{2}}^{a, b, c}(\cdot, n+1)\right\}$ is a basis of $\mathcal{S}$. Moreover, when the boundary value problem is regular, then there is a unique resolvent kernel, a unique Green kernel and a unique Poisson kernel, which are determined by fixing the second variable and finding the unique solution of the previous boundary problems. The importance of these kernels is shown in the following result.

Proposition 5 If the boundary value problem $\left(\mathcal{L}_{q}^{\gamma}, \mathfrak{c}_{1}, \mathfrak{c}_{2}\right)$ is regular, then

$$
R_{\mathfrak{c}_{1}, \mathfrak{c}_{2}}^{a, b}=G_{\mathfrak{c}_{1}, \mathfrak{c}_{2}}^{a, b}+P_{\mathfrak{c}_{1}, \mathfrak{c}_{2}}^{a, b} .
$$

Moreover, for any $f \in \mathcal{C}(\mathbf{I})$ the function

$$
v(k)=\sum_{s \in \mathbf{I}} G_{\mathfrak{c}_{1}, \mathfrak{c}_{2}}^{a, b, c}(k, s) f(s)=\sum_{s=1}^{n} G_{\mathfrak{c}_{1}, \mathfrak{c}_{2}}^{a, b, c}(k, s) f(s), \quad k \in \mathbf{I},
$$

is the unique solution of the semi-homogeneous boundary problem

$$
\mathcal{L}_{q}^{\gamma}(v)=f \quad \text { on } \quad \stackrel{\circ}{\mathbf{I}}, \quad \mathfrak{c}_{1}(v)=\mathfrak{c}_{2}(v)=0,
$$

the function

$$
z(k)=\sum_{s \in \delta(\mathbf{I})} P_{\mathfrak{c}_{1}, \mathfrak{c}_{2}}^{a, b, c}(k, s) f(s)=P_{\mathfrak{c}_{1}, \mathfrak{c}_{2}}^{a, b, c}(k, 0) f(0)+P_{\mathfrak{c}_{1}, \mathfrak{c}_{2}}^{a, b, c}(k, n+1) f(n+1), \quad k \in \mathbf{I},
$$

is the unique solution of the boundary value problem

$$
\mathcal{L}_{q}^{\gamma}(v)=0 \quad \text { on } \quad \stackrel{\circ}{\mathbf{I}}, \quad \mathfrak{c}_{1}(v)=f(0), \quad \mathfrak{c}_{2}(v)=f(n+1)
$$

and, the function $u=v+z$, that is,

$$
u(k)=\sum_{s \in \mathbf{I}} R_{\mathfrak{c}_{1}, \mathfrak{c}_{2}}^{a, b, c}(k, s) f(s)=\sum_{s=0}^{n+1} R_{\mathfrak{c}_{1}, \mathfrak{c}_{2}}^{a, b, c}(k, s) f(s), \quad k \in \mathbf{I},
$$

is the unique solution of the boundary value problem with data $f$

$$
\mathcal{L}_{q}^{\gamma}(v)=f \quad \text { on } \quad \stackrel{\circ}{\mathbf{I}}, \quad \mathfrak{c}_{1}(v)=f(0), \quad \mathfrak{c}_{2}(v)=f(n+1) .
$$


Proof The properties for the function $z$ are immediate. On the other hand, since

$$
v(k)=\sum_{s=1}^{n} G_{\mathfrak{c}_{1}, \mathfrak{c}_{2}}^{a, b, c}(k, s) f(s) \text { for any } k \in \mathbf{I},
$$

then

$$
\mathfrak{c}_{1}(v)=\sum_{s=1}^{n} \mathfrak{c}_{1}\left(G_{\mathfrak{c}_{1}, \mathfrak{c}_{2}}^{a, b, c}(\cdot, s)\right) f(s)=0 \quad \text { and } \quad \mathfrak{c}_{2}(v)=\sum_{s=1}^{n} \mathfrak{c}_{2}\left(G_{\mathfrak{c}_{1}, \mathfrak{c}_{2}}^{a, b, c}(\cdot, s)\right) f(s)=0
$$

and, moreover, for each $k \in \stackrel{\circ}{\mathbf{I}}$

$$
\mathcal{L}_{q}^{\gamma}(v)(k)=\sum_{s=1}^{n} \mathcal{L}_{q}^{\gamma}\left(G_{\mathfrak{c}_{1}, \mathfrak{c}_{2}}^{a, b, c}(\cdot, s)\right)(k) f(s)=\sum_{s=1}^{n} \varepsilon_{s}(k) f(s)=f(k),
$$

such that $\mathcal{L}_{q}^{\gamma}(v)=f$ en $\stackrel{\circ}{\mathbf{I}}$. Then, the properties for the function $u$ are an immediate consequence.

\section{Computation of Poisson, Green and resolvent kernels}

The main results of this section are the computation of the Green, Poisson and resolvent kernels for each regular boundary value problem.

Our main result proves that all of them are totally determined by suitable solutions of the homogeneous Schrödinger equation and the coefficients of the boundary conditions. To the best of the authors knowledge, this property is only known in the case of Sturm-Liouville conditions. Therefore, our methodology appears as a novelty and also could be used to the computation of these kernels in the differentiable case.

Throughout the section we keep the notation of the previous ones. So, we consider fixed the functions $b \in \mathcal{C}(\mathbf{I}), a, c \in \mathcal{C}^{*}(\mathbf{I})$ such that $a(n+1)=c(n)$ and $c(n+1)=a(n)$, the conductance $\gamma \in \Gamma(\mathbf{I})$ and the potential $q \in \mathcal{C}(\mathbf{I})$ determined by them and hence the corresponding Schrödinger operator, $\mathcal{L}_{q}^{\gamma}$, and its corresponding Green function that is denoted by $g$. Moreover, $\mathcal{S}$ denotes the space of solutions of the homogeneous Schrödinger equation $\mathcal{L}_{q}^{\gamma}(u)=0$ on $\stackrel{\circ}{\mathbf{I}}$.

In addition, we also consider fixed the pair of boundary conditions $\left(\mathfrak{c}_{1}, \mathfrak{c}_{2}\right)$ determined by the matrix $\mathrm{C}=\left(\sigma_{i j}\right) \in \mathcal{M}_{2 \times 4}(\mathbb{R})$, where we assume that rank $\mathrm{C}=2$, and the values $d_{i j}=\sigma_{1 i} \sigma_{2 j}-\sigma_{2 i} \sigma_{1 j}, 1 \leq i<j \leq 4$.

Associated with the above functions and values, we consider the boundary value problem $\left(\mathcal{L}_{q}^{\gamma}, \mathfrak{c}_{1}, \mathfrak{c}_{2}\right)$ and $D_{\mathfrak{c}_{1}, \mathfrak{c}_{2}}^{a, b,}$ its determinant. When this problem is regular, our objective is to compute the kernels $G_{\mathfrak{c}_{1}, \mathfrak{c}_{2}}^{a, b}, P_{\mathfrak{c}_{1}, \mathfrak{c}_{2}}^{a, b}$ and then $R_{\mathfrak{c}_{1}, \mathfrak{c}_{2}}^{a, b, c}$.

Definition 11 Let us consider $\nu_{1}, \nu_{2}, \mu_{1}, \mu_{2} \in \mathcal{C}(\mathbf{I})$ the unique solutions of the homogeneous Schrödinger equation on $\stackrel{\circ}{\mathbf{I}}$ determined by the conditions

$$
\begin{aligned}
& \nu_{1}(0)=-\sigma_{12}, \quad \nu_{1}(1)=\sigma_{11}, \quad \nu_{2}(n)=-\sigma_{14}, \quad \nu_{2}(n+1)=\sigma_{13}, \\
& \mu_{1}(0)=-\sigma_{22}, \quad \mu_{1}(1)=\sigma_{21}, \quad \mu_{2}(n)=-\sigma_{24}, \quad \mu_{2}(n+1)=\sigma_{23} \text {. }
\end{aligned}
$$


We call fundamental solutions of the homogeneous Schrödinger equation on $\stackrel{\circ}{\mathbf{I}}$, related to the boundary conditions $\mathfrak{c}_{1}$ and $\mathfrak{c}_{2}$ or, simply, fundamental solutions to the functions

$$
\phi_{\mathfrak{c}_{1}, \mathfrak{c}_{2}}^{a, b}=a(n) \rho_{\gamma}(n) \nu_{1}+a(0) \nu_{2} \quad \text { and } \quad \psi_{\mathfrak{c}_{1}, \mathfrak{c}_{2}}^{a, b, c}=a(n) \rho_{\gamma}(n) \mu_{1}+a(0) \mu_{2} .
$$

Notice that $\mu_{1}, \nu_{1}$ are solutions of initial value problems, whereas $\mu_{2}, \nu_{2}$ are solutions of final value problems, both related with the homogeneous Schrödinger equation. Therefore, all of them depend only on the coefficients $a, b$ and $c$.

The reason to choose these definitions for the fundamental solutions is shown in the following result.

Proposition 6 If $\phi_{\mathfrak{c}_{1}, \mathfrak{c}_{2}}^{a, b, c}$ and $\psi_{\mathfrak{c}_{1}, \mathfrak{c}_{2}}^{a, b}$ are the fundamental solutions of the homogeneous Schrödinger equation on $\stackrel{\circ}{\mathbf{I}}$, related to the boundary conditions $\mathfrak{c}_{1}$ and $\mathfrak{c}_{2}$, then

$$
\mathfrak{c}_{1}\left(\phi_{\mathfrak{c}_{1}, \mathfrak{c}_{2}}^{a, b, c}\right)=\mathfrak{c}_{2}\left(\psi_{\mathfrak{c}_{1}, \mathfrak{c}_{2}}^{a, b}\right)=0, \quad-\mathfrak{c}_{1}\left(\psi_{\mathfrak{c}_{1}, \mathfrak{c}_{2}}^{a, b, c}\right)=\mathfrak{c}_{2}\left(\phi_{\mathfrak{c}_{1}, \mathfrak{c}_{2}}^{a, b, c}\right)=D_{\mathfrak{c}_{1}, \mathfrak{c}_{2}}^{a, b}
$$

and, moreover,

$$
W\left[\phi_{\mathfrak{c}_{1}, \mathfrak{c}_{2}}^{a, b}, \psi_{\mathfrak{c}_{1}, \mathfrak{c}_{2}}^{a, b, c}\right]=\left(D_{\mathfrak{c}_{1}, \mathfrak{c}_{2}}^{a, b, c}\right)^{2} \quad \text { and } \quad w\left[\phi_{\mathfrak{c}_{1}, \mathfrak{c}_{2}}^{a, b}, \psi_{\mathfrak{c}_{1}, \mathfrak{c}_{2}}^{a, b}\right](0)=a(n) \rho_{\gamma}(n) D_{\mathfrak{c}_{1}, \mathfrak{c}_{2}}^{a, b, c}
$$

Proof Let $\{u, v\}$ be the basis of solutions of the homogeneous Schrödinger equation satisfying $u(0)=1, u(1)=0, v(0)=0$ and $v(1)=1$, that is, $u=c(0) g(\cdot, 1)$ and $v=-a(0) g(\cdot, 0)$. Therefore, $w[u, v](0)=1$, which implies that $w[u, v](n)=$ $a(0) a(n)^{-1} \rho_{\gamma}(n)^{-1}$, whereas

$$
W[u, v]=-a(0) c(0) W[g(\cdot, 1), g(\cdot, 0)]=a(n)^{-1} \rho_{\gamma}(n)^{-1} D_{\mathfrak{c}_{1}, \mathfrak{c}_{2}}^{a, b} .
$$

If we prove that

$$
\phi_{\mathfrak{c}_{1}, \mathfrak{c}_{2}}^{a, b, c}=a(n) \rho_{\gamma}(n)\left(\mathfrak{c}_{1}(u) v-\mathfrak{c}_{1}(v) u\right) \quad \text { and } \quad \psi_{\mathfrak{c}_{1}, \mathfrak{c}_{2}}^{a, b}=a(n) \rho_{\gamma}(n)\left(\mathfrak{c}_{2}(u) v-\mathfrak{c}_{2}(v) u\right),
$$

then, $\mathfrak{c}_{1}\left(\phi_{\mathfrak{c}_{1}, \mathfrak{c}_{2}}^{a, b, c}\right)=\mathfrak{c}_{2}\left(\psi_{\mathfrak{c}_{1}, \mathfrak{c}_{2}}^{a, b, c}\right)=0$ and

$$
-\mathfrak{c}_{1}\left(\psi_{\mathfrak{c}_{1}, \mathfrak{c}_{2}}^{a, b, c}\right)=\mathfrak{c}_{2}\left(\phi_{\mathfrak{c}_{1}, \mathfrak{c}_{2}}^{a, b, c}\right)=a(n) \rho_{\gamma}(n) W[u, v]=D_{\mathfrak{c}_{1}, \mathfrak{c}_{2}}^{a, b, c} .
$$

Moreover, since $w$ is skew-symmetric we obtain

$$
w\left[\phi_{\mathbf{c}_{1}, \mathfrak{c}_{2}}^{a, b, c}, \psi_{\mathbf{c}_{1}, \mathfrak{c}_{2}}^{a, b}\right](0)=a(n)^{2} \rho_{\gamma}(n)^{2} W[u, v] w[u, v](0)=a(n) \rho_{\gamma}(n) D_{\mathfrak{c}_{1}, \mathfrak{c}_{2}}^{a, b, c}
$$

and also

$$
W\left[\phi_{\mathfrak{c}_{1}, \mathfrak{c}_{2}}^{a, b}, \psi_{\mathbf{c}_{1}, \mathfrak{c}_{2}}^{a, b, c}\right]=a(n)^{2} \rho_{\gamma}(n)^{2} W[u, v]^{2}=\left(D_{\mathfrak{c}_{1}, \mathfrak{c}_{2}}^{a, b, c}\right)^{2} .
$$

To verify the given expressions for $\phi_{\mathfrak{c}_{1}, \mathfrak{c}_{2}}^{a, b, c}$ and $\psi_{\mathfrak{c}_{1}, \mathfrak{c}_{2}}^{a, b, c}$, let us first observe that $\mathfrak{c}_{1}(u) v-\mathfrak{c}_{1}(v) u=z+x$, where $z$ and $x$ are the solutions of the homogeneous Schrödinger equation on $\stackrel{\circ}{\mathbf{I}}$ defined as

$$
\begin{aligned}
& z=\sigma_{11} v-\sigma_{12} u \\
& x=\sigma_{13}(u(n) v-v(n) u)+\sigma_{14}(u(n+1) v-v(n+1) u) .
\end{aligned}
$$

Since

$$
\begin{aligned}
z(0) & =-\sigma_{12}, & z(1) & =\sigma_{11} \\
x(n) & =-\sigma_{14} w[u, v](n), & x(n+1) & =\sigma_{13} w[u, v](n),
\end{aligned}
$$


we conclude that $z=\nu_{1}$ and $x=w[u, v](n) \nu_{2}=a(0) a(n)^{-1} \rho_{\gamma}(n)^{-1} \nu_{2}$.

On the other hand, $\mathfrak{c}_{2}(u) v-\mathfrak{c}_{2}(v) u=\hat{z}+\hat{x}$, where $\hat{z}, \hat{x} \in \mathcal{S}$ are defined as

$$
\begin{aligned}
& \hat{z}=\sigma_{21} v-\sigma_{22} u \\
& \hat{x}=\sigma_{23}(u(n) v-v(n) u)+\sigma_{24}(u(n+1) v-v(n+1) u) .
\end{aligned}
$$

Since

$$
\begin{aligned}
\hat{z}(0) & =-\sigma_{22}, & \hat{z}(1) & =\sigma_{21} \\
\hat{x}(n) & =-\sigma_{24} w[u, v](n), & \hat{x}(n+1) & =\sigma_{23} w[u, v](n),
\end{aligned}
$$

we conclude that $\hat{z}=\mu_{1}$ and $\hat{x}=w[u, v](n) \mu_{2}=a(0) a(n)^{-1} \rho_{\gamma}(n)^{-1} \mu_{2}$.

Corollary 2 The boundary value problem $\left(\mathcal{L}_{q}^{\gamma}, \mathfrak{c}_{1}, \mathfrak{c}_{2}\right)$ is regular if and only if the fundamental solutions form a basis of solutions of the homogeneous Schrödinger equation on $\stackrel{\circ}{\mathbf{I}}$. Moreover, when the problem is regular, if $u \in \mathcal{C}(\mathbf{I})$ is a solution of the homogeneous Schrödinger equation on $\stackrel{\circ}{\mathbf{I}}$, then $\mathfrak{c}_{1}(u)=0$ if and only if $u=\lambda \phi_{\mathfrak{c}_{1}, \mathfrak{c}_{2}}^{a, b, c}$, and analogously $\mathfrak{c}_{2}(u)=0$ if and only if $u=\lambda \psi_{\mathfrak{c}_{1}, \mathfrak{c}_{2}}^{a, b, c}$, where $\lambda \in \mathbb{R}$.

Proof The first statement comes from Proposition 4 together with Proposition 6 and taking into account that two solutions of the homogeneous Schrödinger equation are linearly independent if and only if its Wronskian is non null at some vertex of $\mathbf{I}$.

When the problem is regular, then $u=\lambda_{1} \phi_{\mathfrak{c}_{1}, \mathfrak{c}_{2}}^{a, b, c}+\lambda_{2} \psi_{\mathfrak{c}_{1}, \mathfrak{c}_{2}}^{a, b}, \lambda_{1}, \lambda_{2} \in \mathbb{R}$, determines all functions $u \in \mathcal{C}(\mathbf{I})$ such that $\mathcal{L}_{q}^{\gamma}(u)=0$. From Proposition 6 , we have that $\mathfrak{c}_{1}(u)=-\lambda_{2} D_{\mathfrak{c}_{1}, \mathfrak{c}_{2}}^{a, b, c}$, whereas $\mathfrak{c}_{2}(u)=\lambda_{1} D_{\mathfrak{c}_{1}, \mathfrak{c}_{2}}^{a, b, c}$. Therefore, $\mathfrak{c}_{1}(u)=0$ if and only if $\lambda_{2}=0$ and $\mathfrak{c}_{2}(u)=0$ if and only if $\lambda_{1}=0$.

The above result motivates that when the boundary value problem $\left(\mathcal{L}_{q}^{\gamma}, \mathfrak{c}_{1}, \mathfrak{c}_{2}\right)$ is regular, we call fundamental basis to $\left\{\phi_{\mathbf{c}_{1}, \mathbf{c}_{2}}^{a, b, c}, \psi_{\mathbf{c}_{1}, \mathfrak{c}_{2}}^{a, b}\right\}$. So, in what follows, the fundamental basis refers to this specific basis of the space $\mathcal{S}$.

Definition 12 Let $\left(\mathcal{L}_{q}^{\gamma}, \mathfrak{c}_{1}, \mathfrak{c}_{2}\right)$ be a regular boundary value problem and consider $\left\{\phi_{\mathfrak{c}_{1}, \mathfrak{c}_{2}}^{a, b, c}, \psi_{\mathfrak{c}_{1}, \mathfrak{c}_{2}}^{a, b}\right\}$ its fundamental basis. The parameters of the boundary value problem are the following real numbers:

$$
\begin{array}{ll}
g_{11}=\sigma_{11} \phi_{\mathfrak{c}_{1}, \mathfrak{c}_{2}}^{a, b, c}(0)+\sigma_{12} \phi_{\mathfrak{c}_{1}, \mathfrak{c}_{2}}^{a, b}(1), & g_{12}=\sigma_{13} \psi_{\mathbf{c}_{1}, \mathfrak{c}_{2}}^{a, b, c}(n)+\sigma_{14} \psi_{\mathfrak{c}_{1}, \mathfrak{c}_{2}}^{a, b, c}(n+1), \\
g_{21}=\sigma_{21} \phi_{\mathfrak{c}_{1}, \mathfrak{c}_{2}}^{a, b, c}(0)+\sigma_{22} \phi_{\mathfrak{c}_{1}, \mathfrak{c}_{2}}^{a, b, c}(1), & g_{22}=\sigma_{23} \psi_{\mathfrak{c}_{1}, \mathfrak{c}_{2}}^{a, b}(n)+\sigma_{24} \psi_{\mathfrak{c}_{1}, \mathfrak{c}_{2}}^{a, b}(n+1) .
\end{array}
$$

Lemma 6 Let $\left(\mathcal{L}_{q}^{\gamma}, \mathfrak{c}_{1}, \mathfrak{c}_{2}\right)$ be a regular boundary value problem. Then, the parameters of the boundary value problem satisfy the following properties:

$$
\begin{aligned}
& g_{11}=-\left[\sigma_{13} \phi_{\mathfrak{c}_{1}, \mathfrak{c}_{2}}^{a, b, c}(n)+\sigma_{14} \phi_{\mathfrak{c}_{1}, \mathfrak{c}_{2}}^{a, b}(n+1)\right], \\
& g_{12}=-D_{\mathfrak{c}_{1}, \mathfrak{c}_{2}}^{a, b}-\left[\sigma_{11} \psi_{\mathfrak{c}_{1}, \mathfrak{c}_{2}}^{a, b, c}(0)+\sigma_{12} \psi_{\mathfrak{c}_{1}, \mathfrak{c}_{2}}^{a, b}(1)\right], \\
& g_{21}=D_{\mathfrak{c}_{1}, \mathfrak{c}_{2}}^{a, b}-\left[\sigma_{23} \phi_{\mathfrak{c}_{1}, \mathfrak{c}_{2}}^{a, b}(n)+\sigma_{24} \phi_{\mathfrak{c}_{1}, \mathfrak{c}_{2}}^{a, b}(n+1)\right], \\
& g_{22}=-\left[\sigma_{21} \psi_{\mathfrak{c}_{1}, \mathfrak{c}_{2}}^{a, b, c}(0)+\sigma_{22} \psi_{\mathfrak{c}_{1}, \mathfrak{c}_{2}}^{a, b}(1)\right] .
\end{aligned}
$$

Moreover, $g_{12}+g_{21}=a(n) \rho_{\gamma}(n) d_{12}-a(0) d_{34}$ and

$g_{11} g_{22}-g_{12} g_{21}=g_{21} D_{\mathfrak{c}_{1}, \mathfrak{c}_{2}}^{a, b, c}-d_{12} w\left[\phi_{\mathfrak{c}_{1}, \mathfrak{c}_{2}}^{a, b, c}, \psi_{\mathfrak{c}_{1}, \mathfrak{c}_{2}}^{a, b, c}\right](0)=-g_{12} D_{\mathfrak{c}_{1}, \mathfrak{c}_{2}}^{a, b, c}-d_{34} w\left[\phi_{\mathfrak{c}_{1}, \mathfrak{c}_{2}}^{a, b, c}, \psi_{\mathfrak{c}_{1}, \mathfrak{c}_{2}}^{a, b, c}\right](n)$. 
Proof For the first identities, it suffices to take into account the following equalities of Proposition 6

$$
\begin{aligned}
0 & =\mathfrak{c}_{1}\left(\phi_{\mathfrak{c}_{1}, \mathfrak{c}_{2}}^{a, b, c}\right)=g_{11}+\sigma_{13} \phi_{\mathfrak{c}_{1}, \mathfrak{c}_{2}}^{a, b}(n)+\sigma_{14} \phi_{\mathfrak{c}_{1}, \mathfrak{c}_{2}}^{a, b, c}(n+1), \\
D_{\mathfrak{c}_{1}, \mathfrak{c}_{2}}^{a, b} & =\mathfrak{c}_{2}\left(\phi_{\mathfrak{c}_{1}, \mathfrak{c}_{2}}^{a, b, c}\right)=g_{21}+\sigma_{23} \phi_{\mathfrak{c}_{1}, \mathfrak{c}_{2}}^{a, b, c}(n)+\sigma_{24} \phi_{\mathfrak{c}_{1}, \mathfrak{c}_{2}}^{a, b, c}(n+1), \\
-D_{\mathfrak{c}_{1}, \mathfrak{c}_{2}}^{a, b} & =\mathfrak{c}_{1}\left(\psi_{\mathfrak{c}_{1}, \mathfrak{c}_{2}}^{a, b, c}\right)=g_{12}+\sigma_{11} \psi_{\mathfrak{c}_{1}, \mathfrak{c}_{2}}^{a, b}(0)+\sigma_{12} \psi_{\mathfrak{c}_{1}, \mathfrak{c}_{2}}^{a, b}(1), \\
0 & =\mathfrak{c}_{2}\left(\psi_{\mathfrak{c}_{1}, \mathfrak{c}_{2}}^{a, b}\right)=g_{22}+\sigma_{21} \psi_{\mathfrak{c}_{1}, \mathfrak{c}_{2}}^{a, c}(0)+\sigma_{22} \psi_{\mathfrak{c}_{1}, \mathfrak{c}_{2}}^{a, c}(1) .
\end{aligned}
$$

On the other hand,

$$
\begin{aligned}
g_{12} & =w\left[\psi_{\mathbf{c}_{1}, \mathbf{c}_{2}}^{a, b, c}, \nu_{2}\right](n)=a(n) \rho_{\gamma}(n) w\left[\mu_{1}, \nu_{2}\right](n)+a(0) w\left[\mu_{2}, \nu_{2}\right](n) \\
& =a(0) w\left[\mu_{1}, \nu_{2}\right](0)-a(0) d_{34} \\
g_{21} & =w\left[\phi_{\mathbf{c}_{1}, \mathbf{c}_{2}}^{a, b,}, \mu_{1}\right](0)=a(n) \rho_{\gamma}(n) w\left[\nu_{1}, \mu_{1}\right](0)+a(0) w\left[\nu_{2}, \mu_{1}\right](0) \\
& =a(n) \rho_{\gamma}(n) d_{12}-a(0) w\left[\mu_{1}, \nu_{2}\right](0),
\end{aligned}
$$

so $g_{12}+g_{21}=a(n) \rho_{\gamma}(n) d_{12}-a(0) d_{34}$. The proof for the expression $g_{11} g_{22}-g_{12} g_{21}$ is analogous.

Notice that

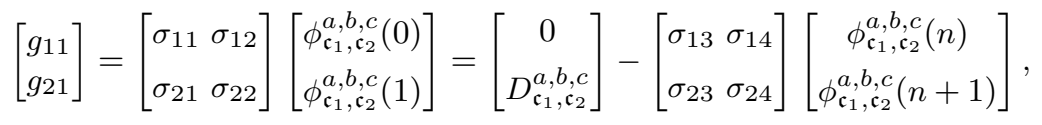

whereas

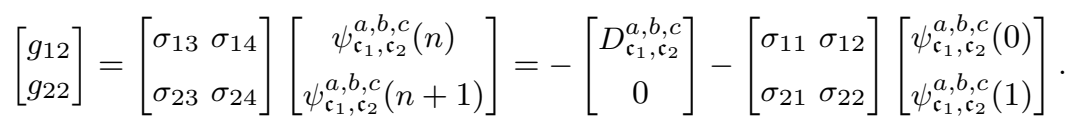

The main result in this section is to express the resolvent, Poisson and Green kernels for a regular boundary value problem in terms of the fundamental basis. In particular, we show that this basis basically determines the Poisson kernel.

Theorem 1 If the boundary value problem $\left(\mathcal{L}_{q}^{\gamma}, \mathfrak{c}_{1}, \mathfrak{c}_{2}\right)$ is regular, the Poisson's kernel is given by the identities

$$
P_{\mathfrak{c}_{1}, \mathfrak{c}_{2}}^{a, b, c}(k, 0)=\frac{-a(0) \psi_{\mathfrak{c}_{1}, \mathfrak{c}_{2}}^{a, b}(k)}{w\left[\phi_{\mathfrak{c}_{1}, \mathfrak{c}_{2}}^{a, b,}, \psi_{\mathfrak{c}_{1}, \mathfrak{c}_{2}}^{a, b}\right](n)} \quad \text { and } \quad P_{\mathfrak{c}_{1}, \mathfrak{c}_{2}}^{a, b}(k, n+1)=\frac{a(0) \phi_{\mathfrak{c}_{1}, \mathfrak{c}_{2}}^{a, b}(k)}{w\left[\phi_{\mathfrak{c}_{1}, \mathfrak{c}_{2}}^{a, b,}, \psi_{\mathfrak{c}_{1}, \mathfrak{c}_{2}}^{a, b, c}\right](n)},
$$

for any $k=0, \ldots, n+1$, whereas the Green's kernel is given by

$$
\begin{aligned}
G_{\mathfrak{c}_{1}, \mathfrak{c}_{2}}^{a, b}(k, s)= & -\frac{\rho_{\gamma}(s) \phi_{\mathfrak{c}_{1}, \mathfrak{c}_{2}}^{a, b, c}(\min \{k, s\}) \psi_{\mathfrak{c}_{1}, \mathfrak{c}_{2}}^{a, b}(\max \{k, s\})}{a(0) w\left[\phi_{\mathfrak{c}_{1}, \mathfrak{c}_{2}}^{a, b}, \psi_{\mathfrak{c}_{1}, \mathfrak{c}_{2}}^{a, b,}\right](0)} \\
& -\frac{\rho_{\gamma}(s)\left[g_{11} \psi_{\mathfrak{c}_{1}, \mathfrak{c}_{2}}^{a, b, c}(k) \psi_{\mathfrak{c}_{1}, \mathfrak{c}_{2}}^{a, b}(s)-g_{22} \phi_{\mathfrak{c}_{1}, \mathfrak{c}_{2}}^{a, b, c}(k) \phi_{\mathfrak{c}_{1}, \mathfrak{c}_{2}}^{a, b, c}(s)\right]}{w\left[\phi_{\mathfrak{c}_{1}, \mathfrak{c}_{2}}^{a, b, c}, \psi_{\mathfrak{c}_{1}, \mathfrak{c}_{2}}^{a, b}\right](0) w\left[\phi_{\mathfrak{c}_{1}, \mathfrak{c}_{2}}^{a, b, c}, \psi_{\mathfrak{c}_{1}, \mathfrak{c}_{2}}^{a, b, c}\right](n)} \\
& -\frac{\rho_{\gamma}(s)\left[g_{12} \psi_{\mathfrak{c}_{1}, \mathfrak{c}_{2}}^{a, b, c}(k) \phi_{\mathfrak{c}_{1}, \mathfrak{c}_{2}}^{a, b}(s)-g_{21} \phi_{\mathfrak{c}_{1}, \mathfrak{c}_{2}}^{a, b}(k) \psi_{\mathfrak{c}_{1}, \mathfrak{c}_{2}}^{a, b}(s)\right]}{w\left[\phi_{\mathfrak{c}_{1}, \mathfrak{c}_{2}}^{a, b}, \psi_{\mathfrak{c}_{1}, \mathfrak{c}_{2}}^{a, b}\right](0) w\left[\phi_{\mathfrak{c}_{1}, \mathfrak{c}_{2}}^{a, b, c}, \psi_{\mathfrak{c}_{1}, \mathfrak{c}_{2}}^{a, b}\right](n)}
\end{aligned}
$$

for any $s=1, \ldots, n$ and any $k=0, \ldots, n+1$. 
Proof Since $\left\{\phi_{\mathbf{c}_{1}, \mathfrak{c}_{2}}^{a, b}, \psi_{\mathfrak{c}_{1}, \mathfrak{c}_{2}}^{a, b, c}\right\}$ is a basis of $\mathcal{S}$, from (7), the Green function of the Schrödinger equation on $\stackrel{\circ}{\mathbf{I}}$ is given by

$$
g(k, s)=g_{00} \rho_{\gamma}(s)\left[\psi_{\mathbf{c}_{1}, \mathfrak{c}_{2}}^{a, b, c}(k) \phi_{\mathbf{c}_{1}, \mathfrak{c}_{2}}^{a, b, c}(s)-\phi_{\mathfrak{c}_{1}, \mathfrak{c}_{2}}^{a, b, c}(k) \psi_{\mathbf{c}_{1}, \mathfrak{c}_{2}}^{a, b, c}(s)\right], \quad k, s \in \mathbf{I} .
$$

where $g_{00}=-\left(a(0) w\left[\phi_{\mathbf{c}_{1}, \mathfrak{c}_{2},}^{a, b, c}, \psi_{\mathbf{c}_{1}, \mathfrak{c}_{2}}^{a, b, c}\right](0)\right)^{-1}$.

Therefore, if $f \in \mathcal{C}(\mathbf{I})$, the set of solutions of the Schrödinger equation $\mathcal{L}_{q}^{\gamma}(u)=f$ on $\stackrel{\circ}{\mathbf{I}}$ is given by the identity

$$
u=\alpha \phi_{\mathfrak{c}_{1}, \mathfrak{c}_{2}}^{a, b,}+\beta \psi_{\mathfrak{c}_{1}, \mathfrak{c}_{2}}^{a, b}+y, \quad \alpha, \beta \in \mathbb{R}
$$

where according with $(8), y(k)=\sum_{s=1}^{k} g(k, s) f(s)$, that is,

$$
y(k)=g_{00} \psi_{\mathfrak{c}_{1}, \mathfrak{c}_{2}}^{a, b, c}(k) \sum_{s=1}^{k} \phi_{\mathfrak{c}_{1}, \mathfrak{c}_{2}}^{a, b, c}(s) \rho_{\gamma}(s) f(s) d s-g_{00} \phi_{\mathfrak{c}_{1}, \mathfrak{c}_{2}}^{a, b, c}(k) \sum_{s=1}^{k} \psi_{\mathfrak{c}_{1}, \mathfrak{c}_{2}}^{a, b, c}(s) \rho_{\gamma}(s) f(s) d s,
$$

is the unique solution of the Schrödinger equation $\mathcal{L}_{q}^{\gamma}(u)=f$ on $\stackrel{\circ}{\mathbf{I}}$ satisfying the initial conditions $y(0)=y(1)=0$.

Using the properties of the fundamental solutions described in Proposition 6, it follows that $\mathfrak{c}_{1}(u)=f(0)$ and $\mathfrak{c}_{2}(u)=f(n+1)$ if and only if

$$
\alpha=\frac{1}{D_{\mathfrak{c}_{1}, \mathfrak{c}_{2}}^{a, b, c}}\left[f(n+1)-\mathfrak{c}_{2}(y)\right] \quad \text { and } \quad \beta=\frac{1}{D_{\mathfrak{c}_{1}, \mathfrak{c}_{2}}^{a, b, c}}\left[\mathfrak{c}_{1}(y)-f(0)\right] .
$$

On the other hand, $\mathfrak{c}_{i}(y)=\sigma_{i 3} y(n)+\sigma_{i 4} y(n+1), i=1,2$ and using the identities from Lemma 6 , it follows that

$$
\begin{aligned}
& \mathfrak{c}_{1}(y)=g_{00} \sum_{s=1}^{n}\left[g_{11} \psi_{\mathfrak{c}_{1}, \mathfrak{c}_{2}}^{a, b, c}(s)+g_{12} \phi_{\mathfrak{c}_{1}, \mathfrak{c}_{2}}^{a, b, c}(s)\right] \rho_{\gamma}(s) f(s) d s, \\
& \mathfrak{c}_{2}(y)=g_{00} \sum_{s=1}^{n}\left[\left(g_{21}-D_{\mathfrak{c}_{1}, \mathfrak{c}_{2}}^{a, b, c}\right) \psi_{\mathfrak{c}_{1}, \mathfrak{c}_{2}}^{a, b, c}(s)+g_{22} \phi_{\mathbf{c}_{1}, \mathfrak{c}_{2}}^{a, b, c}(s)\right] \rho_{\gamma}(s) f(s) d s .
\end{aligned}
$$

To determine the Poisson and Green kernels, we must to substitute the function $f$ in the previous identities by $\varepsilon_{s}, s=0, \ldots, n+1$. Also, note that as $w\left[\phi_{\mathfrak{c}_{1}, \mathfrak{c}_{2}}^{a, b, c}, \psi_{\mathbf{c}_{1}, \mathfrak{c}_{2}}^{a, b}\right](0)=a(n) \rho_{\gamma}(n) D_{\mathfrak{c}_{1}, \mathfrak{c}_{2}}^{a, b}$, then $w\left[\phi_{\mathfrak{c}_{1}, \mathfrak{c}_{2}}^{a, b, c}, \psi_{\mathfrak{c}_{1}, \mathfrak{c}_{2}}^{a, b}\right](n)=a(0) D_{\mathfrak{c}_{1}, \mathfrak{c}_{2}}^{a, b}$.

If we consider $f=\varepsilon_{s}$ where either $s=0$ or $s=n+1$, then $y=0$ which implies that $\mathfrak{c}_{1}(y)=\mathfrak{c}_{2}(y)=0$. Therefore, if $s=0$, then $\alpha=0$ and $\beta=-\frac{1}{D_{\mathfrak{c}_{1}, \mathfrak{c}_{2}}^{a, b, c}}$, whereas if $s=n+1$, then $\beta=0$ and $\alpha=\frac{1}{D_{\mathfrak{c}_{1}, \mathfrak{c}_{2}}^{a, b, c}}$. Definitely, we have obtained that

$$
P_{\mathfrak{c}_{1}, \mathfrak{c}_{2}}^{a, b, c}(k, 0)=-\frac{1}{D_{\mathfrak{c}_{1}, \mathfrak{c}_{2}}^{a, b}} \psi_{\mathfrak{c}_{1}, \mathfrak{c}_{2}}^{a, b}(k) \text { and } P_{\mathfrak{c}_{1}, \mathfrak{c}_{2}}^{a, b, c}(k, n+1)=\frac{1}{D_{\mathfrak{c}_{1}, \mathfrak{c}_{2}}^{a, b,}} \phi_{\mathfrak{c}_{1}, \mathfrak{c}_{2}}^{a, b, c}(k) .
$$

If we consider $f=\varepsilon_{s}, s=1, \ldots, n$, then $\alpha=-\frac{\mathfrak{c}_{2}(y)}{D_{\mathfrak{c}_{1}, \mathfrak{c}_{2}}^{a, b, c}}, \beta=\frac{\mathfrak{c}_{1}(y)}{D_{\mathfrak{c}_{1}, \mathfrak{c}_{2}}^{a, b, c}}$, whereas

$$
y(k)=\left\{\begin{array}{cc}
0, & k \leq s, \\
g_{00} \rho_{\gamma}(s)\left[\psi_{\mathfrak{c}_{1}, \mathfrak{c}_{2}}^{a, b, c}(k) \phi_{\mathfrak{c}_{1}, \mathfrak{c}_{2}}^{a, b}(s)-\phi_{\mathfrak{c}_{1}, \mathfrak{c}_{2}}^{a, b}(k) \psi_{\mathfrak{c}_{1}, \mathfrak{c}_{2}}^{a, b, c}(s)\right], & k \geq s,
\end{array}\right.
$$


or, equivalently,

$$
y(k)=-g_{00} \rho_{\gamma}(s) \phi_{\mathfrak{c}_{1}, \mathfrak{c}_{2}}^{a, b}(k) \psi_{\mathfrak{c}_{1}, \mathfrak{c}_{2}}^{a, b, c}(s)+g_{00} \rho_{\gamma}(s)\left\{\begin{array}{l}
\phi_{\mathfrak{c}_{1}, \mathfrak{c}_{2}}^{a, b}(k) \psi_{\mathfrak{c}_{1}, \mathfrak{c}_{2}}^{a, b}(s), k \leq s, \\
\psi_{\mathfrak{c}_{1}, \mathfrak{c}_{2}}^{a, b}(k) \phi_{\mathfrak{c}_{1}, \mathfrak{c}_{2}}^{a, b}(s), k \geq s,
\end{array}\right.
$$

that is,

$$
y(k)=g_{00} \rho_{\gamma}(s)\left[\phi_{\mathbf{c}_{1}, \mathfrak{c}_{2}}^{a, b}(\min \{k, s\}) \psi_{\mathbf{c}_{1}, \mathfrak{c}_{2}}^{a, b}(\max \{k, s\})-\phi_{\mathfrak{c}_{1}, \mathfrak{c}_{2}}^{a, b, c}(k) \psi_{\mathbf{c}_{1}, \mathfrak{c}_{2}}^{a, b, c}(s)\right] .
$$

On the other hand, we also have

$$
\begin{aligned}
& \mathfrak{c}_{1}(y)=g_{00} \rho_{\gamma}(s)\left[g_{11} \psi_{\mathfrak{c}_{1}, \mathfrak{c}_{2}}^{a, b, c}(s)+g_{12} \phi_{\mathfrak{c}_{1}, \mathfrak{c}_{2}}^{a, b}(s)\right], \\
& \mathfrak{c}_{2}(y)=g_{00} \rho_{\gamma}(s)\left[\left(g_{21}-D_{\mathfrak{c}_{1}, \mathfrak{c}_{2}}^{a, b}\right) \psi_{\mathfrak{c}_{1}, \mathfrak{c}_{2}}^{a, b, c}(s)+g_{22} \phi_{\mathfrak{c}_{1}, \mathfrak{c}_{2}}^{a, b}(s)\right],
\end{aligned}
$$

which implies

$$
\begin{aligned}
G_{\mathfrak{c}_{1}, \mathfrak{c}_{2}}^{a, b, c}(k, s) & =g_{00} \rho_{\gamma}(s)\left[\phi_{\mathfrak{c}_{1}, \mathfrak{c}_{2}}^{a, b}(\min \{k, s\}) \psi_{\mathfrak{c}_{1}, \mathfrak{c}_{2}}^{a, b, c}(\max \{k, s\})-\phi_{\mathfrak{c}_{1}, \mathfrak{c}_{2}}^{a, b}(k) \psi_{\mathfrak{c}_{1}, \mathfrak{c}_{2}}^{a, b, c}(s)\right] \\
& -\frac{g_{00} \rho_{\gamma}(s)}{D_{\mathfrak{c}_{1}, \mathfrak{c}_{2}}^{a, b, c}}\left[\left(g_{21}-D_{\mathfrak{c}_{1}, \mathfrak{c}_{2}}^{a, b, c}\right) \phi_{\mathfrak{c}_{1}, \mathfrak{c}_{2}}^{a, b, c}(k) \psi_{\mathfrak{c}_{1}, \mathfrak{c}_{2}}^{a, b, c}(s)+g_{22} \phi_{\mathfrak{c}_{1}, \mathfrak{c}_{2}}^{a, b, c}(k) \phi_{\mathfrak{c}_{1}, \mathfrak{c}_{2}}^{a, b, c}(s)\right] \\
& +\frac{g_{00} \rho_{\gamma}(s)}{D_{\mathfrak{c}_{1}, \mathfrak{c}_{2}}^{a, b, c}}\left[g_{11} \psi_{\mathfrak{c}_{1}, \mathfrak{c}_{2}}^{a, b, c}(k) \psi_{\mathfrak{c}_{1}, \mathfrak{c}_{2}}^{a, b, c}(s)+g_{12} \psi_{\mathfrak{c}_{1}, \mathfrak{c}_{2}}^{a, b, c}(k) \phi_{\mathfrak{c}_{1}, \mathfrak{c}_{2}}^{a, b, c}(s)\right] \\
& =g_{00} \rho_{\gamma}(s) \phi_{\mathfrak{c}_{1}, \mathfrak{c}_{2}}^{a, b, c}(\min \{k, s\}) \psi_{\mathfrak{c}_{1}, \mathfrak{c}_{2}}^{a, b, c}(\max \{k, s\}) \\
& -\frac{g_{00} \rho_{\gamma}(s)}{D_{\mathfrak{c}_{1}, \mathfrak{c}_{2}}^{a, b, c}}\left[g_{21} \phi_{\mathfrak{c}_{1}, \mathfrak{c}_{2}}^{a, b, c}(k) \psi_{\mathfrak{c}_{1}, \mathfrak{c}_{2}}^{a, b, c}(s)+g_{22} \phi_{\mathfrak{c}_{1}, \mathfrak{c}_{2}}^{a, b, c}(k) \phi_{\mathfrak{c}_{1}, \mathfrak{c}_{2}}^{a, b, c}(s)\right] \\
& +\frac{g_{00} \rho_{\gamma}(s)}{D_{\mathfrak{c}_{1}, \mathfrak{c}_{2}}^{a, b, c}}\left[g_{11} \psi_{\mathfrak{c}_{1}, \mathfrak{c}_{2}}^{a, b, c}(k) \psi_{\mathfrak{c}_{1}, \mathfrak{c}_{2}}^{a, b, c}(s)+g_{12} \psi_{\mathfrak{c}_{1}, \mathfrak{c}_{2}}^{a, b, c}(k) \phi_{\mathfrak{c}_{1}, \mathfrak{c}_{2}}^{a, b, c}(s)\right]
\end{aligned}
$$

Corollary 3 If the boundary value problem $\left(\mathcal{L}_{q}^{\gamma}, \mathfrak{c}_{1}, \mathfrak{c}_{2}\right)$ is regular, then its resolvent kernel is determined by

$$
\begin{aligned}
& R_{\mathfrak{c}_{1}, \mathfrak{c}_{2}}^{a, b, c}(k, s)=-\frac{\rho_{\gamma}(s) \phi_{\mathfrak{c}_{1}, \mathfrak{c}_{2}}^{a, b, c}(\min \{k, s\}) \psi_{\mathfrak{c}_{1}, \mathfrak{c}_{2}}^{a, b}(\max \{k, s\})}{a(0) w\left[\phi_{\mathfrak{c}_{1}, \mathfrak{c}_{2}}^{a, b, c}, \psi_{\mathfrak{c}_{1}, \mathfrak{c}_{2}}^{a, b, c}\right](0)} \\
& -\frac{\rho_{\gamma}(s)\left[g_{11} \psi_{\mathfrak{c}_{1}, \mathfrak{c}_{2}}^{a, b, c}(k) \psi_{\mathfrak{c}_{1}, \mathfrak{c}_{2}}^{a, b, c}(s)-g_{22} \phi_{\mathfrak{c}_{1}, \mathfrak{c}_{2}}^{a, b, c}(k) \phi_{\mathfrak{c}_{1}, \mathfrak{c}_{2}}^{a, b, c}(s)\right]}{w\left[\phi_{\mathfrak{c}_{1}, \mathfrak{c}_{2}}^{a, b,}, \psi_{\mathfrak{c}_{1}, \mathfrak{c}_{2}}^{a, b, c}\right](0) w\left[\phi_{\mathfrak{c}_{1}, \mathfrak{c}_{2}}^{a, b,}, \psi_{\mathfrak{c}_{1}, \mathfrak{c}_{2}}^{a, b, c}\right](n)} \\
& -\frac{\rho_{\gamma}(s)\left[g_{12} \psi_{\mathfrak{c}_{1}, \mathfrak{c}_{2}}^{a, b, c}(k) \phi_{\mathfrak{c}_{1}, \mathfrak{c}_{2}}^{a, b, c}(s)-g_{21} \phi_{\mathfrak{c}_{1}, \mathfrak{c}_{2}}^{a, b}(k) \psi_{\mathfrak{c}_{1}, \mathfrak{c}_{2}}^{a, b, c}(s)\right]}{w\left[\phi_{\mathfrak{c}_{1}, \mathfrak{c}_{2}}^{a, b,}, \psi_{\mathfrak{c}_{1}, \mathfrak{c}_{2}}^{a, b, c}\right](0) w\left[\phi_{\mathfrak{c}_{1}, \mathfrak{c}_{2}}^{a, b,}, \psi_{\mathfrak{c}_{1}, \mathfrak{c}_{2}}^{a, b}\right](n)} \\
& -\frac{\left[\left(\sigma_{12}+a(0)\right) \psi_{\mathbf{c}_{1}, \mathfrak{c}_{2}}^{a, b, c}(k)-\sigma_{22} \phi_{\mathbf{c}_{1}, \mathfrak{c}_{2}}^{a, b}(k)\right]}{w\left[\phi_{\mathfrak{c}_{1}, \mathfrak{c}_{2}}^{a, b, c}, \psi_{\mathfrak{c}_{1}, \mathfrak{c}_{2}}^{a, b, c}\right](n)} \varepsilon_{s}(0) \\
& -\frac{\rho_{\gamma}(n+1)\left[\sigma_{13} \psi_{\mathfrak{c}_{1}, \mathfrak{c}_{2}}^{a, b}(k)-\left(c(n)+\sigma_{23}\right) \phi_{\mathfrak{c}_{1}, \mathfrak{c}_{2}}^{a, b, c}(k)\right]}{w\left[\phi_{\mathfrak{c}_{1}, \mathfrak{c}_{2}}^{a, b}, \psi_{\mathfrak{c}_{1}, \mathfrak{c}_{2}}^{a, b, c}\right](0)} \varepsilon_{s}(n+1),
\end{aligned}
$$

for any $k, s=0, \ldots, n+1$. 
Proof As $R_{\mathfrak{c}_{1}, \mathfrak{c}_{2}}^{a, b, c}=G_{\mathfrak{c}_{1}, \mathfrak{c}_{2}}^{a, b}+P_{\mathfrak{c}_{1}, \mathfrak{c}_{2}}^{a, b}$, the equality holds for any $k=0, \ldots, n+1$, when $s=1, \ldots, n$, so the identity given for $R_{\mathfrak{c}_{1}, \mathfrak{c}_{2}}^{a, b}$ corresponds to that obtained for the Green kernel in Theorem 1.

Let us consider now $A_{0}, A_{n+1} \in \mathcal{C}(\mathbf{I})$ the functions obtained allowing $s=0$ and $s=n+1$ in the Green kernel expression, that is,

$$
\begin{aligned}
& A_{0}(k)=-\frac{\phi_{\mathfrak{c}_{1}, \mathfrak{c}_{2}}^{a, b, c}(0) \psi_{\mathfrak{c}_{1}, \mathfrak{c}_{2}}^{a, b}(k)}{a(0) w\left[\phi_{\mathfrak{c}_{1}, \mathfrak{c}_{2}}^{a, b}, \psi_{\mathfrak{c}_{1}, \mathfrak{c}_{2}}^{a, b, c}\right](0)}-\frac{\left(g_{11} \psi_{\mathfrak{c}_{1}, \mathfrak{c}_{2}}^{a, b, c}(0)+g_{12} \phi_{\mathfrak{c}_{1}, \mathfrak{c}_{2}}^{a, b}(0)\right) \psi_{\mathfrak{c}_{1}, \mathfrak{c}_{2}}^{a, b, c}(k)}{w\left[\phi_{\mathfrak{c}_{1}, \mathfrak{c}_{2}}^{a, b}, \psi_{\mathfrak{c}_{1}, \mathfrak{c}_{2}}^{a, c}\right](0) w\left[\phi_{\mathfrak{c}_{1}, \mathfrak{c}_{2}}^{a, b, c}, \psi_{\mathfrak{c}_{1}, \mathfrak{c}_{2}}^{a, b}\right](n)} \\
& +\frac{\left(g_{22} \phi_{\mathfrak{c}_{1}, \mathfrak{c}_{2}}^{a, b, c}(0)+g_{21} \psi_{\mathfrak{c}_{1}, \mathfrak{c}_{2}}^{a, b, c}(0)\right) \phi_{\mathfrak{c}_{1}, \mathfrak{c}_{2}}^{a, b}(k)}{w\left[\phi_{\mathfrak{c}_{1}, \mathfrak{c}_{2}}^{a, b, c}, \psi_{\mathfrak{c}_{1}, \mathfrak{c}_{2}}^{a, b, c}\right](0) w\left[\phi_{\mathfrak{c}_{1}, \mathfrak{c}_{2}}^{a, b,}, \psi_{\mathfrak{c}_{1}, \mathfrak{c}_{2}}^{a, b, c}\right](n)} \\
& =-\frac{\left(g_{11} \psi_{\mathfrak{c}_{1}, \mathfrak{c}_{2}}^{a, b, c}(0)+\left(g_{12}+D_{\mathfrak{c}_{1}, \mathfrak{c}_{2}}^{a, b, c}\right) \phi_{\mathfrak{c}_{1}, \mathfrak{c}_{2}}^{a, b}(0)\right) \psi_{\mathfrak{c}_{1}, \mathfrak{c}_{2}}^{a, b, c}(k)}{w\left[\phi_{\mathbf{c}_{1}, \mathfrak{c}_{2}}^{a, b}, \psi_{\mathfrak{c}_{1}, \mathfrak{c}_{2}}^{a, b}\right](0) w\left[\phi_{\mathfrak{c}_{1}, \mathfrak{c}_{2}}^{a, c}, \psi_{\mathfrak{c}_{1}, \mathfrak{c}_{2}}^{a, b}\right](n)} \\
& +\frac{\left(g_{22} \phi_{\mathfrak{c}_{1}, \mathfrak{c}_{2}}^{a, b, c}(0)+g_{21} \psi_{\mathfrak{c}_{1}, \mathfrak{c}_{2}}^{a, b, c}(0)\right) \phi_{\mathfrak{c}_{1}}^{a, \mathfrak{c}_{2}}(k)}{w\left[\phi_{\mathfrak{c}_{1}, \mathfrak{c}_{2}}^{a, b}, \psi_{\mathbf{c}_{1}, \mathfrak{c}_{2}}^{a, c}\right](0) w\left[\phi_{\mathbf{c}_{1}, \mathfrak{c}_{2}}^{a, b}, \psi_{\mathfrak{c}_{1}, \mathfrak{c}_{2}}^{a, c}\right](n)}
\end{aligned}
$$

and

$$
\begin{aligned}
& A_{n+1}(k)=-\frac{\rho_{\gamma}(n+1) \phi_{\mathfrak{c}_{1}, \mathfrak{c}_{2}}^{a, b, c}(k) \psi_{\mathfrak{c}_{1}, \mathfrak{c}_{2}}^{a, b}(n+1)}{a(0) w\left[\phi_{\mathfrak{c}_{1}, \mathfrak{c}_{2}}^{a, b, c}, \psi_{\mathfrak{c}_{1}, \mathfrak{c}_{2}}^{a, b}\right](0)} \\
& -\frac{\rho_{\gamma}(n+1)\left(g_{11} \psi_{\mathfrak{c}_{1}, \mathfrak{c}_{2}}^{a, b, c}(n+1)+g_{12} \phi_{\mathfrak{c}_{1}, \mathfrak{c}_{2}}^{a, b, c}(n+1)\right) \psi_{\mathfrak{c}_{1}, \mathfrak{c}_{2}}^{a, b, c}(k)}{w\left[\phi_{\mathbf{c}_{1}, \mathfrak{c}_{2}}^{a, c}, \psi_{\mathfrak{c}_{1}, \mathfrak{c}_{2}}^{a, b, c}\right](0) w\left[\phi_{\mathfrak{c}_{1}, \mathfrak{c}_{2}}^{a, b}, \psi_{\mathfrak{c}_{1}, \mathfrak{c}_{2}}^{a, b}\right](n)} \\
& +\frac{\rho_{\gamma}(n+1)\left(g_{22} \phi_{\mathfrak{c}_{1}}^{a, b, \mathfrak{c}_{2}}(n+1)+g_{21} \psi_{\mathfrak{c}_{1}, \mathfrak{c}_{2}}^{a, b}(n+1)\right) \phi_{\mathfrak{c}_{1}, \mathfrak{c}_{2}}^{a, b, c}(k)}{w\left[\phi_{\mathfrak{c}_{1}, \mathfrak{c}_{2}}^{a, b}, \psi_{\mathfrak{c}_{1}, \mathfrak{c}_{2}}^{a, b}\right](0) w\left[\phi_{\mathfrak{c}_{1}, \mathfrak{c}_{2}}^{a, b}, \psi_{\mathfrak{c}_{1}, \mathfrak{c}_{2}}^{a, c}\right](n)} \\
& =-\frac{\rho_{\gamma}(n+1)\left(g_{11} \psi_{\mathfrak{c}_{1}, \mathfrak{c}_{2}}^{a, b, c}(n+1)+g_{12} \phi_{\mathfrak{c}_{1}, \mathfrak{c}_{2}}^{a, b, c}(n+1)\right) \psi_{\mathfrak{c}_{1}, \mathfrak{c}_{2}}^{a, b, c}(k)}{w\left[\phi_{\mathfrak{c}_{1}, \mathfrak{c}_{2}}^{a, b,}, \psi_{\mathfrak{c}_{1}, \mathfrak{c}_{2}}^{a, b, c}\right](0) w\left[\phi_{\mathfrak{c}_{1}, \mathfrak{c}_{2}}^{a, b,}, \psi_{\mathfrak{c}_{1}, \mathfrak{c}_{2}}^{a, b}\right](n)} \\
& +\frac{\rho_{\gamma}(n+1)\left(g_{22} \phi_{\mathfrak{c}_{1}, \mathfrak{c}_{2}}^{a, b, c}(n+1)+\left(g_{21}-D_{\mathfrak{c}_{1}, \mathfrak{c}_{2}}^{a, b}\right) \psi_{\mathfrak{c}_{1}, \mathfrak{c}_{2}}^{a, b, c}(n+1)\right) \phi_{\mathfrak{c}_{1}, \mathfrak{c}_{2}}^{a, b, c}(k)}{w\left[\phi_{\mathfrak{c}_{1}, \mathfrak{c}_{2}}^{a, b, c}, \psi_{\mathfrak{c}_{1}, \mathfrak{c}_{2}}^{a, b, c}\right](0) w\left[\phi_{\mathfrak{c}_{1}, \mathfrak{c}_{2}}^{a, b}, \psi_{\mathfrak{c}_{1}, \mathfrak{c}_{2}}^{a, b}\right](n)},
\end{aligned}
$$

where it has been used that $D_{\mathfrak{c}_{1}, \mathfrak{c}_{2}}^{a, b, c}=a(0)^{-1} w\left[\phi_{\mathfrak{c}_{1}, \mathfrak{c}_{2}}^{a, b}, \psi_{\mathbf{c}_{1}, \mathfrak{c}_{2}}^{a, b}\right](n)$. Taking into account the identities from Definition 12 and Lemma 6, it follows

$$
\begin{aligned}
g_{11} \psi_{\mathfrak{c}_{1}, \mathfrak{c}_{2}}^{a, b}(0)+\left(g_{12}+D_{\mathfrak{c}_{1}, \mathfrak{c}_{2}}^{a, b}\right) \phi_{\mathfrak{c}_{1}, \mathfrak{c}_{2}}^{a, b}(0) & =-\sigma_{12} w\left[\phi_{\mathfrak{c}_{1}, \mathfrak{c}_{2}}^{a, b,}, \psi_{\mathfrak{c}_{1}, \mathfrak{c}_{2}}^{a, b, c}\right](0), \\
g_{21} \psi_{\mathfrak{c}_{1}, \mathfrak{c}_{2}}^{a, b}(0)+g_{22} \phi_{\mathfrak{c}_{1}, \mathfrak{c}_{2}}^{a, b}(0) & =-\sigma_{22} w\left[\phi_{\mathfrak{c}_{1}, \mathfrak{c}_{2}}^{a, b}, \psi_{\mathfrak{c}_{1}, \mathfrak{c}_{2}}^{a, b}\right](0), \\
\left(g_{21}-D_{\mathfrak{c}_{1}, \mathfrak{c}_{2}}^{a, b}\right) \psi_{\mathfrak{c}_{1}, \mathfrak{c}_{2}}^{a, b}(n+1)+g_{22} \phi_{\mathfrak{c}_{1}, \mathfrak{c}_{2}}^{a, b}(n+1) & =-\sigma_{23} w\left[\phi_{\mathfrak{c}_{1}, \mathfrak{c}_{2}}^{a, b}, \psi_{\mathfrak{c}_{1}, \mathfrak{c}_{2}}^{a, b}\right](n), \\
g_{11} \psi_{\mathfrak{c}_{1}, \mathfrak{c}_{2}}^{a, b}(n+1)+g_{12} \phi_{\mathfrak{c}_{1}, \mathfrak{c}_{2}}^{a, b}(n+1) & =-\sigma_{13} w\left[\phi_{\mathfrak{c}_{1}, \mathfrak{c}_{2}}^{a, b, c}, \psi_{\mathfrak{c}_{1}, \mathfrak{c}_{2}}^{a, b}\right](n),
\end{aligned}
$$

which implies

$$
\begin{aligned}
A_{0}(k) & =\frac{\sigma_{12} \psi_{\mathfrak{c}_{1}}^{a, \mathfrak{c}_{2}}(k)}{w\left[\phi_{\mathfrak{c}_{1}, \mathfrak{c}_{2}}^{a, b}, \psi_{\mathfrak{c}_{1}, \mathfrak{c}_{2}}^{a, b, c}\right](n)}-\frac{\sigma_{22} \phi_{\mathbf{c}_{1}, \mathfrak{c}_{2}}^{a, b, c}(k)}{w\left[\phi_{\mathfrak{c}_{1}, \mathfrak{c}_{2}}^{a, b,}, \psi_{\mathbf{c}_{1}, \mathfrak{c}_{2}}^{a, b, c}\right](n)} \\
& =P_{\mathfrak{c}_{1}, \mathfrak{c}_{2}}^{a, b, c}(k, 0)+\frac{\left(a(0)+\sigma_{12}\right) \psi_{\mathfrak{c}_{1}, \mathfrak{c}_{2}}^{a, b}(k)-\sigma_{22} \phi_{\mathfrak{c}_{1}, \mathfrak{c}_{2}}^{a, b, c}(k)}{w\left[\phi_{\mathfrak{c}_{1}, \mathfrak{c}_{2}}^{a, b}, \psi_{\mathfrak{c}_{1}, \mathfrak{c}_{2}}^{a, b}\right](n)}
\end{aligned}
$$


and also that

$$
\begin{aligned}
A_{n+1}(k) & =\frac{\rho_{\gamma}(n+1)\left[\sigma_{13} \psi_{\mathfrak{c}_{1}, \mathfrak{c}_{2}}^{a, b, c}(k)-\sigma_{23} \phi_{\mathfrak{c}_{1}, \mathfrak{c}_{2}}^{a, b, c}(k)\right]}{w\left[\phi_{\mathfrak{c}_{1}, \mathfrak{c}_{2}}^{a, b, c}, \psi_{\mathfrak{c}_{1}, \mathfrak{c}_{2}}^{a, c}\right](0)} \\
& =P_{\mathfrak{c}_{1}, \mathfrak{c}_{2}}^{a, b, c}(k, n+1)-\frac{a(0) \phi_{\mathfrak{c}_{1}, \mathfrak{c}_{2}}^{a, b}(k)}{w\left[\phi_{\mathfrak{c}_{1}, \mathfrak{c}_{2}}^{a, b,}, \psi_{\mathfrak{c}_{1}, \mathfrak{c}_{2}}^{a, b, c}\right](n)} \\
& +\frac{\rho_{\gamma}(n+1)\left[\sigma_{13} \psi_{\mathfrak{c}_{1}, \mathfrak{c}_{2}}^{a, b, c}(k)-\sigma_{23} \phi_{\mathfrak{c}_{1}, \mathfrak{c}_{2}}^{a, b, c}(k)\right]}{w\left[\phi_{\mathfrak{c}_{1}, \mathfrak{c}_{2}}^{a, b, c}, \psi_{\mathfrak{c}_{1}, \mathfrak{c}_{2}}^{a, c}\right](0)} \\
& =P_{\mathfrak{c}_{1}, \mathfrak{c}_{2}}^{a, b, c}(k, n+1)+\frac{\rho_{\gamma}(n+1)\left[\left(\sigma_{13} \psi_{\mathfrak{c}_{1}, \mathfrak{c}_{2}}^{a, b}(k)-\left(\sigma_{23}+c(n)\right) \phi_{\mathfrak{c}_{1}, \mathfrak{c}_{2}}^{a, b}(k)\right]\right.}{w\left[\phi_{\mathfrak{c}_{1}, \mathfrak{c}_{2}}^{a, b}, \psi_{\mathfrak{c}_{1}, \mathfrak{c}_{2}}^{a, b}\right](0)},
\end{aligned}
$$

where it has been taking into account $a(n) \rho_{\gamma}(n)=c(n) \rho_{\gamma}(n+1)$.

\section{Resolvent kernels of regular boundary value problems}

The purpose of this section is to analyze the different boundary value problems according to the classification we made in Section 3. Therefore, we study unilateral problems, either initial or final, separable problems either at one or at both extremes of the path and finally, periodic problems. In all cases, we establish the specific conditions for such problems to be regular and we determine the corresponding resolvent kernel. Since Green and Poisson kernels can be immediately deduced from the expression of the resolvent kernel, we do not write down the expressions for these last kernels.

\subsection{Unilateral problems}

Let us consider, first, initial unilateral boundary conditions $\left(\mathfrak{c}_{1}, \mathfrak{c}_{2}\right)$, that is, such that $\mathrm{C}_{34}=0$ and hence $d_{12} \neq 0$.

In this case, $\phi_{\mathfrak{c}_{1}, \mathfrak{c}_{2}}^{a, b}$ is the unique solution of the homogeneous Schrödinger equation on $\stackrel{\circ}{\mathbf{I}}$ that satisfies $\phi_{\mathbf{c}_{1}, \mathbf{c}_{2}}^{a, b, c}(0)=-a(n) \rho_{\gamma}(n) \sigma_{12}$ and $\phi_{\mathbf{c}_{1}, \mathbf{c}_{2}}^{a, b, c}(1)=a(n) \rho_{\gamma}(n) \sigma_{11}$, whereas $\psi_{\mathfrak{c}_{1}, \mathfrak{c}_{2}}^{a, b}$ is the unique solution of the homogeneous Schrödinger equation on $\stackrel{\circ}{\mathbf{I}}$ such that $\psi_{\mathfrak{c}_{1}, \mathfrak{c}_{2}}^{a, b, c}(0)=-a(n) \rho_{\gamma}(n) \sigma_{22}$ and $\psi_{\mathfrak{c}_{1}, \mathfrak{c}_{2}}^{a, b, c}(1)=a(n) \rho_{\gamma}(n) \sigma_{21}$. Since

$$
w\left[\phi_{\mathfrak{c}_{1}, \mathfrak{c}_{2}}^{a, b, c}, \psi_{\mathbf{c}_{1}, \mathfrak{c}_{2}}^{a, b, c}\right](0)=a(n)^{2} \rho_{\gamma}(n)^{2} d_{12} \neq 0,
$$

the problem is regular. Moreover, $g_{11}=g_{22}=g_{12}=0$, whereas

$$
g_{21}=a(n) \rho_{\gamma}(n) d_{12}=a(0)^{-1} w\left[\phi_{\mathbf{c}_{1}, \mathfrak{c}_{2}}^{a, b, c}, \psi_{\mathbf{c}_{1}, \mathfrak{c}_{2}}^{a, b, c}\right](n) .
$$

Therefore, we obtain the following result. 
Proposition 7 Let $\left(\mathcal{L}_{q}^{\gamma}, \mathfrak{c}_{1}, \mathfrak{c}_{2}\right)$ be a initial unilateral problem, then it is regular and its resolvent kernel is given by

$$
\begin{aligned}
R_{\mathfrak{c}_{1}, \mathfrak{c}_{2}}^{a, b, c}(k, s) & =\frac{\rho_{\gamma}(s)\left[\phi_{\mathfrak{c}_{1}, \mathfrak{c}_{2}}^{a, b, c}(k) \psi_{\mathfrak{c}_{1}, \mathfrak{c}_{2}}^{a, b, c}(s)-\phi_{\mathfrak{c}_{1}, \mathfrak{c}_{2}}^{a, b}(\min \{k, s\}) \psi_{\mathfrak{c}_{1}, \mathfrak{c}_{2}}^{a, b, c}(\max \{k, s\})\right]}{a(0) w\left[\phi_{\mathfrak{c}_{1}, \mathfrak{c}_{2}}^{a, b}, \psi_{\mathfrak{c}_{1}, \mathfrak{c}_{2}}^{a, b}\right](0)} \\
& -\frac{\left[\left(\sigma_{12}+a(0)\right) \psi_{\mathfrak{c}_{1}, \mathfrak{c}_{2}}^{a, b}(k)-\sigma_{22} \phi_{\mathfrak{c}_{1}, \mathfrak{c}_{2}}^{a, b}(k)\right]}{w\left[\phi_{\mathfrak{c}_{1}, \mathfrak{c}_{2}}^{a, b,}, \psi_{\mathfrak{c}_{1}, \mathfrak{c}_{2}}^{a, b}\right](n)} \varepsilon_{s}(0) \\
& +\frac{\rho_{\gamma}(n+1) c(n) \phi_{\mathfrak{c}_{1}, \mathfrak{c}_{2}}^{a, b}(k)}{w\left[\phi_{\mathfrak{c}_{1}, \mathfrak{c}_{2}}^{a, b}, \psi_{\mathfrak{c}_{1}, \mathfrak{c}_{2}}^{a, b, c}\right](0)} \varepsilon_{s}(n+1),
\end{aligned}
$$

for any $k, s=0, \ldots, n+1$.

Let us consider now final unilateral boundary condition $\left(\mathfrak{c}_{1}, \mathfrak{c}_{2}\right)$, that is, such that $\mathrm{C}_{12}=0$ and hence $d_{34} \neq 0$.

In this case, $\phi_{\mathfrak{c}_{1}, \mathfrak{c}_{2}}^{a, b, c}$ is the unique solution of the homogeneous Schrödinger equation on $\stackrel{\circ}{\mathbf{I}}$ that verifies $\phi_{\mathbf{c}_{1}, \mathfrak{c}_{2}}^{a, b, c}(n)=-a(0) \sigma_{14}$ and $\phi_{\mathbf{c}_{1}, \mathbf{c}_{2}}^{a, b, c}(n+1)=a(0) \sigma_{13}$, whereas $\psi_{\mathbf{c}_{1}, \mathfrak{c}_{2}}^{a, b, c}$ is the unique solution of the homogeneous Schrödinger equation on $\stackrel{\circ}{\mathbf{I}}$ such that $\psi_{\mathfrak{c}_{1}, \mathfrak{c}_{2}}^{a, b}(n)=-a(0) \sigma_{24}$ and $\psi_{\mathfrak{c}_{1}, \mathfrak{c}_{2}}^{a, b, c}(n+1)=a(0) \sigma_{23}$. Since

$$
w\left[\phi_{\mathbf{c}_{1}, \mathfrak{c}_{2}}^{a, b}, \psi_{\mathbf{c}_{1}, \mathfrak{c}_{2}}^{a, b, c}\right](n)=a(0)^{2} d_{34} \neq 0,
$$

the problem is regular. Moreover, $g_{11}=g_{22}=g_{21}=0$, whereas $g_{12}=-a(0) d_{34}$. Therefore, we obtain the following result.

Proposition $\mathbf{8}$ Let $\left(\mathcal{L}_{q}^{\gamma}, \mathfrak{c}_{1}, \mathfrak{c}_{2}\right)$ be a final unilateral problem, then it is regular and its resolvent kernel is given by

$$
\begin{aligned}
R_{\mathfrak{c}_{1}, \mathfrak{c}_{2}}^{a, b, c}(k, s) & =\frac{\rho_{\gamma}(s)\left[\psi_{\mathbf{c}_{1}, \mathfrak{c}_{2}}^{a, b, c}(k) \phi_{\mathbf{c}_{1}, \mathfrak{c}_{2}}^{a, b, c}(s)-\phi_{\mathbf{c}_{1}, \mathfrak{c}_{2}}^{a, b}(\min \{k, s\}) \psi_{\mathfrak{c}_{1}, \mathfrak{c}_{2}}^{a, b, c}(\max \{k, s\})\right]}{a(0) w\left[\phi_{\mathbf{c}_{1}, \mathfrak{c}_{2}}^{a, b, c}, \psi_{\mathfrak{c}_{1}, \mathfrak{c}_{2}}^{a, b}\right](0)} \\
& -\frac{a(0) \psi_{\mathfrak{c}_{1}, \mathfrak{c}_{2}}^{a, b, c}(k)}{w\left[\phi_{\mathfrak{c}_{1}, \mathfrak{c}_{2}}^{a, b,}, \psi_{\mathfrak{c}_{1}}^{a, \mathfrak{c}_{2}}\right](n)} \varepsilon_{s}(0) \\
& -\frac{\rho_{\gamma}(n+1)\left[\sigma_{13} \psi_{\mathfrak{c}_{1}, \mathfrak{c}_{2}}^{a, b, c}(k)-\left(c(n)+\sigma_{23}\right) \phi_{\mathfrak{c}_{1}, \mathfrak{c}_{2}}^{a, b, c}(k)\right]}{w\left[\phi_{\mathfrak{c}_{1}, \mathfrak{c}_{2}}^{a, b, c}, \psi_{\mathfrak{c}_{1}, \mathfrak{c}_{2}}^{a, b, c}(0)\right.} \varepsilon_{s}(n+1),
\end{aligned}
$$

for each $k, s=0, \ldots, n+1$

Note that in the case of initial unilateral boundary conditions,

$$
\begin{aligned}
G_{\mathfrak{c}_{1}, \mathfrak{c}_{2}}^{a, b, c}(k, s) & =\frac{\rho_{\gamma}(s)\left[\phi_{\mathbf{c}_{1}, \mathfrak{c}_{2}}^{a, b, c}(k) \psi_{\mathbf{c}_{1}, \mathfrak{c}_{2}}^{a, b, c}(s)-\phi_{\mathbf{c}_{1}, \mathfrak{c}_{2}}^{a, b}(\min \{k, s\}) \psi_{\mathbf{c}_{1}, \mathfrak{c}_{2}}^{a, b, c}(\max \{k, s\})\right]}{a(0) w\left[\phi_{\mathbf{c}_{1}, \mathfrak{c}_{2}}^{a, b}, \psi_{\mathfrak{c}_{1}, \mathfrak{c}_{2}}^{a, b,}\right](0)} \\
& =\left\{\begin{array}{c}
0, \quad \text { si } k \leq s, \\
-g(k, s), \text { si } k \geq s,
\end{array}\right.
\end{aligned}
$$


while when we consider final unilateral boundary conditions,

$$
\begin{aligned}
G_{\mathfrak{c}_{1}, \mathfrak{c}_{2}}^{a, b, c}(k, s) & =\frac{\rho_{\gamma}(s)\left[\psi_{\mathbf{c}_{1}, \mathfrak{c}_{2}}^{a, b, c}(k) \phi_{\mathbf{c}_{1}, \mathfrak{c}_{2}}^{a, b, c}(s)-\phi_{\mathfrak{c}_{1}, \mathfrak{c}_{2}}^{a, b, c}(\min \{k, s\}) \psi_{\mathbf{c}_{1}, \mathfrak{c}_{2}}^{a, b, c}(\max \{k, s\})\right]}{a(0) w\left[\phi_{\mathfrak{c}_{1}, \mathfrak{c}_{2}}^{a, b,}, \psi_{\mathfrak{c}_{1}, \mathfrak{c}_{2}}^{a, b, c}\right](0)} \\
& =\left\{\begin{array}{c}
g(k, s), \text { si } k \leq s, \\
0, \quad \text { si } k \geq s,
\end{array}\right.
\end{aligned}
$$

where $g$ is the Green function of the Schrödinger equation on $\stackrel{\circ}{\mathbf{I}}$.

\subsection{Separable problems}

Let us consider the boundary conditions pair $\left(\mathfrak{c}_{1}, \mathfrak{c}_{2}\right)$ separable at 0 and more specifically, that it is determined by the matrix $\left[\begin{array}{cccc}\sigma_{11} & \sigma_{12} & 0 & 0 \\ \sigma_{21} & \sigma_{22} & \sigma_{23} & \sigma_{24}\end{array}\right]$, where it is satisfy that $\left(\left|\sigma_{11}\right|+\left|\sigma_{12}\right|\right)\left(\left|\sigma_{23}\right|+\left|\sigma_{24}\right|\right)>0$ and $\sigma_{21} \cdot \sigma_{22}=0$.

In this case, $\phi_{\mathfrak{c}_{1}, \mathfrak{c}_{2}}^{a, b}$ is the unique solution of the homogeneous Schrödinger equation on $\stackrel{\circ}{\mathbf{I}}$ that verifies $\phi_{\mathbf{c}_{1}, \mathfrak{c}_{2}}^{a, b}(0)=-a(n) \rho_{\gamma}(n) \sigma_{12}$ and $\phi_{\mathbf{c}_{1}, \mathfrak{c}_{2}}^{a, b, c}(1)=a(n) \rho_{\gamma}(n) \sigma_{11}$. Since

$$
w\left[\phi_{\mathbf{c}_{1}, \mathfrak{c}_{2}}^{a, b, c}, \psi_{\mathbf{c}_{1}, \mathfrak{c}_{2}}^{a, b, c}\right](0)=-a(n) \rho_{\gamma}(n)\left[\sigma_{11} \psi_{\mathfrak{c}_{1}, \mathfrak{c}_{2}}^{a, b, c}(0)+\sigma_{12} \psi_{\mathfrak{c}_{1}, \mathfrak{c}_{2}}^{a, b, c}(1)\right]
$$

then $D_{\mathfrak{c}_{1}, \mathfrak{c}_{2}}^{a, b, c}=-\left[\sigma_{11} \psi_{\mathfrak{c}_{1}, \mathfrak{c}_{2}}^{a, b, c}(0)+\sigma_{12} \psi_{\mathfrak{c}_{1}, \mathfrak{c}_{2}}^{a, b, c}(1)\right]$. Moreover, $g_{11}=g_{12}=0, g_{21}=$ $a(n) \rho_{\gamma}(n) d_{12}$ and $w\left[\phi_{\mathfrak{c}_{1}, \mathfrak{c}_{2}}^{a, b}, \psi_{\mathfrak{c}_{1}, \mathfrak{c}_{2}}^{a, b, c}\right](n)=-a(0)\left[\sigma_{11} \psi_{\mathfrak{c}_{1}, \mathfrak{c}_{2}}^{a, b}(0)+\sigma_{12} \psi_{\mathfrak{c}_{1}, \mathfrak{c}_{2}}^{a, b, c}(1)\right]$.

Proposition 9 Let $\left(\mathcal{L}_{q}^{\gamma}, \mathfrak{c}_{1}, \mathfrak{c}_{2}\right)$ be a boundary value problem separable at 0 , then it is regular if and only if $\sigma_{11} \psi_{\mathfrak{c}_{1}, \mathfrak{c}_{2}}^{a, b}(0) \neq-\sigma_{12} \psi_{\mathfrak{c}_{1}, \mathfrak{c}_{2}}^{a, b}(1)$ and its resolvent kernel is given by

$$
\begin{aligned}
R_{\mathfrak{c}_{1}, \mathfrak{c}_{2}}^{a, b, c}(k, s) & =\frac{\rho_{\gamma}(s) \phi_{\mathfrak{c}_{1}, \mathfrak{c}_{2}}^{a, b}(\min \{k, s\}) \psi_{\mathfrak{c}_{1}, \mathfrak{c}_{2}}^{a, b, c}(\max \{k, s\})}{a(0) a(n) \rho_{\gamma}(n)\left[\sigma_{11} \psi_{\mathfrak{c}_{1}, \mathfrak{c}_{2}}^{a, b, c}(0)+\sigma_{12} \psi_{\mathfrak{c}_{1}, \mathfrak{c}_{2}}^{a, b, c}(1)\right]} \\
& +\frac{\rho_{\gamma}(s)\left[\left(g_{22} \phi_{\mathfrak{c}_{1}, \mathfrak{c}_{2}}^{a, b, c}(s)+d_{12} a(n) \rho_{\gamma}(n) \psi_{\mathfrak{c}_{1}, \mathfrak{c}_{2}}^{a, b, c}(s)\right) \phi_{\mathfrak{c}_{1}, \mathfrak{c}_{2}}^{a, b}(k)\right]}{a(0) a(n) \rho_{\gamma}(n)\left[\sigma_{11} \psi_{\mathfrak{c}_{1}, \mathfrak{c}_{2}}^{a, b, c}(0)+\sigma_{12} \psi_{\mathfrak{c}_{1}, \mathfrak{c}_{2}}^{a, b}(1)\right]^{2}} \\
& +\frac{\left[\left(a(0)+\sigma_{12}\right) \psi_{\mathfrak{c}_{1}, \mathfrak{c}_{2}}^{a, b, c}(k)-\sigma_{22} \phi_{\mathfrak{c}_{1}, \mathfrak{c}_{2}}^{a, c}(k)\right]}{a(0)\left[\sigma_{11} \psi_{\mathfrak{c}_{1}, \mathfrak{c}_{2}}^{a, b}(0)+\sigma_{12} \psi_{\mathfrak{c}_{1}, \mathfrak{c}_{2}}^{a, b, c}(1)\right]} \varepsilon_{s}(0) \\
& -\frac{\left(\sigma_{23}+c(n)\right) \phi_{\mathfrak{c}_{1}, \mathfrak{c}_{2}}^{a, b}(k)}{c(n)\left[\sigma_{11} \psi_{\mathfrak{c}_{1}, \mathfrak{c}_{2}}^{a, b, c}(0)+\sigma_{12} \psi_{\mathfrak{c}_{1}, \mathfrak{c}_{2}}^{a, b}(1)\right]} \varepsilon_{s}(n+1),
\end{aligned}
$$

for each $k, s=0, \ldots, n+1$.

Let us consider the boundary conditions pair $\left(\mathfrak{c}_{1}, \mathfrak{c}_{2}\right)$ separable at $n+1$ and it is determined by the matrix $\left[\begin{array}{cccc}\sigma_{11} & \sigma_{12} & \sigma_{13} & \sigma_{14} \\ 0 & 0 & \sigma_{23} & \sigma_{24}\end{array}\right]$, where $\left(\left|\sigma_{11}\right|+\left|\sigma_{12}\right|\right)\left(\left|\sigma_{23}\right|+\left|\sigma_{24}\right|\right)>0$ and $\sigma_{13} \cdot \sigma_{14}=0$. 
In this case, $\psi_{\mathfrak{c}_{1}, \mathfrak{c}_{2}}^{a, b, c}$ is the unique solution of the homogeneous Schrödinger equation on $\stackrel{\circ}{\mathbf{I}}$ that verifies $\psi_{\mathfrak{c}_{1}, \mathfrak{c}_{2}}^{a, b, c}(n)=-a(0) \sigma_{24}$ and $\psi_{\mathfrak{c}_{1}, \mathfrak{c}_{2}}^{a, b, c}(n+1)=a(0) \sigma_{23}$. Since

$$
w\left[\phi_{\mathfrak{c}_{1}, \mathfrak{c}_{2}}^{a, b}, \psi_{\mathbf{c}_{1}, \mathfrak{c}_{2}}^{a, b}\right](n)=a(0)\left[\sigma_{23} \phi_{\mathfrak{c}_{1}, \mathfrak{c}_{2}}^{a, b, c}(n)+\sigma_{24} \phi_{\mathfrak{c}_{1}, \mathfrak{c}_{2}}^{a, b}(n+1)\right]
$$

then $D_{\mathfrak{c}_{1}, \mathfrak{c}_{2}}^{a, b, c}=\sigma_{23} \phi_{\mathfrak{c}_{1}, \mathfrak{c}_{2}}^{a, b, c}(n)+\sigma_{24} \phi_{\mathfrak{c}_{1}, \mathfrak{c}_{2}}^{a, b, c}(n+1)$. Moreover, $g_{21}=g_{22}=0, g_{12}=$ $-a(0) d_{34}$ and $w\left[\phi_{\mathfrak{c}_{1}, \mathfrak{c}_{2}}^{a, b}, \psi_{\mathfrak{c}_{1}, \mathfrak{c}_{2}}^{a, b, c}\right](0)=a(n) \rho_{\gamma}(n)\left[\sigma_{23} \phi_{\mathfrak{c}_{1}, \mathfrak{c}_{2}}^{a, b}(n)+\sigma_{24} \phi_{\mathfrak{c}_{1}, \mathfrak{c}_{2}}^{a, b, c}(n+1)\right]$.

Proposition 10 Let $\left(\mathcal{L}_{q}^{\gamma}, \mathfrak{c}_{1}, \mathfrak{c}_{2}\right)$ be the boundary value problem separable at $n+1$, then it is regular if and only if $\sigma_{23} \phi_{\mathfrak{c}_{1}, \mathfrak{c}_{2}}^{a, b, c}(n) \neq-\sigma_{24} \phi_{\mathfrak{c}_{1}, \mathfrak{c}_{2}}^{a, b, c}(n+1)$ and its resolvent kernel is given by

$$
\begin{aligned}
R_{\mathfrak{c}_{1}, \mathfrak{c}_{2}}^{a, b, c}(k, s)= & -\frac{\rho_{\gamma}(s) \phi_{\mathfrak{c}_{1}, \mathfrak{c}_{2}}^{a, b, c}(\min \{k, s\}) \psi_{\mathfrak{c}_{1}, \mathfrak{c}_{2}}^{a, b, c}(\max \{k, s\})}{a(0) a(n) \rho_{\gamma}(n)\left[\sigma_{23} \phi_{\mathfrak{c}_{1}, \mathfrak{c}_{2}}^{a, b}(n)+\sigma_{24} \phi_{\mathfrak{c}_{1}, \mathfrak{c}_{2}}^{a, b, c}(n+1)\right]} \\
& -\frac{\rho_{\gamma}(s)\left[g_{11} \psi_{\mathfrak{c}_{1}, \mathfrak{c}_{2}}^{a, b, c}(s)-a(0) d_{34} \phi_{\mathfrak{c}_{1}, \mathfrak{c}_{2}}^{a, b, c}(s)\right] \psi_{\mathfrak{c}_{1}, \mathfrak{c}_{2}}^{a, b}(k)}{a(0) a(n) \rho_{\gamma}(n)\left[\sigma_{23} \phi_{\mathfrak{c}_{1}, \mathfrak{c}_{2}}^{a, b, c}(n)+\sigma_{24} \phi_{\mathfrak{c}_{1}, \mathfrak{c}_{2}}^{a, b, c}(n+1)\right]^{2}} \\
& -\frac{\left(\sigma_{12}+a(0)\right) \psi_{\mathfrak{c}_{1}, \mathfrak{c}_{2}}^{a, b}(k)}{a(0)\left[\sigma_{23} \phi_{\mathfrak{c}_{1}, \mathfrak{c}_{2}}^{a, b, c}(n)+\sigma_{24} \phi_{\mathfrak{c}_{1}, \mathfrak{c}_{2}}^{a, b, c}(n+1)\right]} \varepsilon_{s}(0) \\
& -\frac{\left[\sigma_{13} \psi_{\mathfrak{c}_{1}, \mathfrak{c}_{2}}^{a, b}(k)-\left(c(n)+\sigma_{23}\right) \phi_{\mathfrak{c}_{1}, \mathfrak{c}_{2}}^{a, b}(k)\right]}{c(n)\left[\sigma_{23} \phi_{\mathfrak{c}_{1}, \mathfrak{c}_{2}}^{a, b, c}(n)+\sigma_{24} \phi_{\mathbf{c}_{1}, \mathfrak{c}_{2}}^{a, b}(n+1)\right]} \varepsilon_{s}(n+1),
\end{aligned}
$$

for each $k, s=0, \ldots, n+1$.

Let us consider, finally, the Sturm-Liouville pair $\left(\mathfrak{c}_{1}, \mathfrak{c}_{2}\right)$, that is determined by the matrix $\left[\begin{array}{cccc}\sigma_{11} & \sigma_{12} & 0 & 0 \\ 0 & 0 & \sigma_{23} & \sigma_{24}\end{array}\right]$, where $\left(\left|\sigma_{11}\right|+\left|\sigma_{12}\right|\right)\left(\left|\sigma_{23}+\right| \sigma_{24} \mid\right)>0$.

In that case, $\phi_{\mathbf{c}_{1}, \mathfrak{c}_{2}}^{a, b, c}$ is the unique solution of the homogeneous Schrödinger equation on $\stackrel{\circ}{\mathbf{I}}$ that verifies $\phi_{\mathbf{c}_{1}, \mathfrak{c}_{2}}^{a, b, c}(0)=-a(n) \rho_{\gamma}(n) \sigma_{12}$ and $\phi_{\mathbf{c}_{1}, \mathfrak{c}_{2}}^{a, b, c}(1)=a(n) \rho_{\gamma}(n) \sigma_{11}$, whereas $\psi_{\mathfrak{c}_{1}, \mathfrak{c}_{2}}^{a, b}$ is the unique solution of the homogeneous Schrödinger equation on $\stackrel{\circ}{\mathbf{I}}$ such that $\psi_{\mathfrak{c}_{1}, \mathfrak{c}_{2}}^{a, b}(n)=-a(0) \sigma_{24}$ and $\psi_{\mathfrak{c}_{1}, \mathfrak{c}_{2}}^{a, b}(n+1)=a(0) \sigma_{23}$. Moreover, $g_{11}=$ $g_{12}=g_{21}=g_{22}=0$ and

$$
D_{\mathfrak{c}_{1}, \mathfrak{c}_{2}}^{a, b, c}=-\sigma_{11} \psi_{\mathfrak{c}_{1}, \mathfrak{c}_{2}}^{a, b, c}(0)-\sigma_{12} \psi_{\mathfrak{c}_{1}, \mathfrak{c}_{2}}^{a, b, c}(1)=\sigma_{23} \phi_{\mathfrak{c}_{1}, \mathfrak{c}_{2}}^{a, b}(n)+\sigma_{24} \phi_{\mathfrak{c}_{1}, \mathfrak{c}_{2}}^{a, b, c}(n+1) .
$$

Proposition 11 The Sturm-Liouville boundary value problem $\left(\mathcal{L}_{q}^{\gamma}, \mathfrak{c}_{1}, \mathfrak{c}_{2}\right)$ is regular if and only if $\sigma_{11} \psi_{\mathfrak{c}_{1}, \mathfrak{c}_{2}}^{a, b, c}(0) \neq-\sigma_{12} \psi_{\mathfrak{c}_{1}, \mathfrak{c}_{2}}^{a, b}(1)$ or, equivalently, if and only if $\sigma_{23} \phi_{\mathfrak{c}_{1}, \mathfrak{c}_{2}}^{a, b, c}(n) \neq$ $-\sigma_{24} \phi_{\mathfrak{c}_{1}, \mathfrak{c}_{2}}^{a, b, c}(n+1)$ and its resolvent kernel is determined by

$$
\begin{aligned}
R_{\mathfrak{c}_{1}, \mathfrak{c}_{2}}^{a, b, c}(k, s)= & -\frac{\rho_{\gamma}(s) \phi_{\mathfrak{c}_{1}, \mathfrak{c}_{2}}^{a, b, c}(\min \{k, s\}) \psi_{\mathfrak{c}_{1}, \mathfrak{c}_{2}}^{a, b}(\max \{k, s\})}{a(0) a(n) \rho_{\gamma}(n)\left[\sigma_{23} \phi_{\mathfrak{c}_{1}, \mathfrak{c}_{2}}^{a, b}(n)+\sigma_{24} \phi_{\mathfrak{c}_{1}, \mathfrak{c}_{2}}^{a, b, c}(n+1)\right]} \\
& -\frac{\left(\sigma_{12}+a(0)\right) \psi_{\mathfrak{c}_{1}, \mathfrak{c}_{2}}^{a, b, c}(k)}{a(0)\left[\sigma_{23} \phi_{\mathfrak{c}_{1}, \mathfrak{c}_{2}}^{a, b}(n)+\sigma_{24} \phi_{\mathfrak{c}_{1}, \mathfrak{c}_{2}}^{a, b, c}(n+1)\right]} \varepsilon_{s}(0) \\
& +\frac{\left(c(n)+\sigma_{23}\right) \phi_{\mathfrak{c}_{1}, \mathfrak{c}_{2}}^{a, b, c}(k)}{c(n)\left[\sigma_{23} \phi_{\mathfrak{c}_{1}, \mathfrak{c}_{2}}^{a, b, c}(n)+\sigma_{24} \phi_{\mathfrak{c}_{1}, \mathfrak{c}_{2}}^{a, b, c}(n+1)\right]} \varepsilon_{s}(n+1),,
\end{aligned}
$$

for any $k, s=0, \ldots, n+1$. 
Note that for Sturm-Liouville problems, the Green kernel can be expressed as

$$
G_{\mathfrak{c}_{1}, \mathfrak{c}_{2}}^{a, b, c}(k, s)=-\frac{\rho_{\gamma}(s) \phi_{\mathbf{c}_{1}, \mathfrak{c}_{2}}^{a, b}(\min \{k, s\}) \psi_{\mathfrak{c}_{1}, \mathfrak{c}_{2}}^{a, b, c}(\max \{k, s\})}{a(0) a(n) \rho_{\gamma}(n)\left[\sigma_{23} \phi_{\mathfrak{c}_{1}, \mathfrak{c}_{2}}^{a, b, c}(n)+\sigma_{24} \phi_{\mathfrak{c}_{1}, \mathfrak{c}_{2}}^{a, b, c}(n+1)\right]},
$$

while the resolvent kernel can be expressed as

$$
\begin{aligned}
R_{\mathfrak{c}_{1}, \mathfrak{c}_{2}}^{a, b}(k, s) & =\frac{-\rho_{\gamma}(s)}{a(0) a(n) \rho_{\gamma}(n)\left[\sigma_{23} \phi_{\mathfrak{c}_{1}, \mathfrak{c}_{2}}^{a, b}(n)+\sigma_{24} \phi_{\mathbf{c}_{1}, \mathfrak{c}_{2}}^{a, b}(n+1)\right]} \\
& \times\left[\phi_{\mathfrak{c}_{1}, \mathfrak{c}_{2}}^{a, b, c}(\min \{k, s\})+a(n) \rho_{\gamma}(n)\left(\sigma_{12}+a(0)\right) \varepsilon_{s}(0)\right] \\
& \times\left[\psi_{\mathfrak{c}_{1}, \mathfrak{c}_{2}}^{a, b, c}(\max \{k, s\})-a(0)\left(c(n)+\sigma_{23}\right) \varepsilon_{s}(n+1)\right] .
\end{aligned}
$$

This last identity motivates us to consider a slightly reformulation of the results of the previous proposition, in order to obtain an expression of the resolvent kernel completely analogous to Green kernel expression. For this, we consider the functions

$$
\begin{aligned}
& \Phi_{\mathbf{c}_{1}, \mathfrak{c}_{2}}^{a, b}=\left(a(n) \rho_{\gamma}(n)\right)^{-1} \phi_{\mathfrak{c}_{1}, \mathfrak{c}_{2}}^{a, b, c}+\left(\sigma_{12}+a(0)\right) \varepsilon_{0}, \\
& \Psi_{\mathfrak{c}_{1}, \mathfrak{c}_{2}}^{a, c}=a(0)^{-1} \psi_{\mathbf{c}_{1}, \mathfrak{c}_{2}}^{a, b}-\left(c(n)+\sigma_{23}\right) \varepsilon_{n+1} .
\end{aligned}
$$

Then,

$\mathcal{L}_{q}^{\gamma}\left(\Phi_{\mathfrak{c}_{1}, \mathfrak{c}_{2}}^{a, b}\right)=-c(0)\left(a(0)+\sigma_{12}\right) \varepsilon_{1} \quad$ and $\quad \mathcal{L}_{q}^{\gamma}\left(\Psi_{\mathfrak{c}_{1}, \mathfrak{c}_{2}}^{a, b, c}\right)=a(n)\left(c(n)+\sigma_{23}\right) \varepsilon_{n} \quad$ on $\quad \stackrel{\circ}{\mathbf{I}}$, and, moreover,

$$
\Phi_{\mathfrak{c}_{1}, \mathfrak{c}_{2}}^{a, b, c}(0)=a(0), \quad \Phi_{\mathfrak{c}_{1}, \mathfrak{c}_{2}}^{a, b, c}(1)=\sigma_{11}, \quad \Psi_{\mathfrak{c}_{1}, \mathfrak{c}_{2}}^{a, b, c}(n)=-\sigma_{24}, \quad \Psi_{\mathfrak{c}_{1}, \mathfrak{c}_{2}}^{a, b, c}(n+1)=-c(n) .
$$

Corollary 4 Let us consider the Sturm-Liouville boundary value problem $\left(\mathcal{L}_{q}^{\gamma}, \mathfrak{c}_{1}, \mathfrak{c}_{2}\right)$, and $\Phi_{\mathfrak{c}_{1}, \mathfrak{c}_{2}}^{a, b}$ and $\Psi_{\mathfrak{c}_{1}, \mathfrak{c}_{2}}^{a, b, c}$ the unique solutions of the Schrödinger equation on $\stackrel{\circ}{\mathbf{I}}$ with data $-c(0)\left(a(0)+\sigma_{12}\right) \varepsilon_{1}$ and $a(n)\left(c(n)+\sigma_{23}\right) \varepsilon_{n}$, respectively, that satisfy

$$
\Phi_{\mathfrak{c}_{1}, \mathfrak{c}_{2}}^{a, b, c}(0)=a(0), \quad \Phi_{\mathfrak{c}_{1}, \mathfrak{c}_{2}}^{a, b, c}(1)=\sigma_{11}, \quad \Psi_{\mathfrak{c}_{1}, \mathfrak{c}_{2}}^{a, b, c}(n)=-\sigma_{24}, \quad \Psi_{\mathfrak{c}_{1}, \mathfrak{c}_{2}}^{a, b, c}(n+1)=-c(n) .
$$

Then,

$a(0)\left(\sigma_{11} \Psi_{\mathfrak{c}_{1}, \mathfrak{c}_{2}}^{a, b}(0)+\sigma_{12} \Psi_{\mathfrak{c}_{1}, \mathfrak{c}_{2}}^{a, b, c}(1)\right)=-a(n) \rho_{\gamma}(n)\left(\sigma_{23} \Phi_{\mathfrak{c}_{1}, \mathfrak{c}_{2}}^{a, b, c}(n)+\sigma_{24} \Phi_{\mathfrak{c}_{1}, \mathfrak{c}_{2}}^{a, b}(n+1)\right)$,

the problem is regular if and only if $\sigma_{11} \Psi_{\mathfrak{c}_{1}, \mathfrak{c}_{2}}^{a, b}(0) \neq-\sigma_{12} \Psi_{\mathfrak{c}_{1}, \mathfrak{c}_{2}}^{a, b, c}(1)$ and its resolvent kernel is given by

$$
R_{\mathfrak{c}_{1}, \mathfrak{c}_{2}}^{a, b, c}(k, s)=\frac{\rho_{\gamma}(s)}{a(0)\left[\sigma_{11} \Psi_{\mathfrak{c}_{1}, \mathfrak{c}_{2}}^{a, b, c}(0)+\sigma_{12} \Psi_{\mathfrak{c}_{1}, \mathfrak{c}_{2}}^{a, b, c}(1)\right]} \Phi_{\mathfrak{c}_{1}, \mathfrak{c}_{2}}^{a, b}(\min \{k, s\}) \Psi_{\mathfrak{c}_{1}, \mathfrak{c}_{2}}^{a, b, c}(\max \{k, s\}),
$$

for any $k, s=0, \ldots, n+1$. 
Proof It is enough to take into account that

$$
\Phi_{\mathfrak{c}_{1}, \mathfrak{c}_{2}}^{a, b, c}(k)=\left(a(n) \rho_{\gamma}(n)\right)^{-1} \phi_{\mathfrak{c}_{1}, \mathfrak{c}_{2}}^{a, b, c}(k) \quad \text { and } \quad \Psi_{\mathfrak{c}_{1}, \mathfrak{c}_{2}}^{a, b, c}(k)=a(0)^{-1} \psi_{\mathfrak{c}_{1}, \mathfrak{c}_{2}}^{a, b, c}(k),
$$

for any $k=1, \ldots, n$ and apply the results of Proposition 11.

Next, we will use the expression above to describe the resolvent kernels for the most well-known Sturm-Liouville problems. The general case expressed in Corollary 4, also corresponds to Robin's problem, that is, when both are Robin conditions.

Corollary 5 If the Dirichlet boundary value problem $\left(\mathcal{L}_{q}^{\gamma}, \mathfrak{c}_{1}, \mathfrak{c}_{2}\right)$ is exterior, that is, $\mathfrak{c}_{1}(u)=u(0)$ and $\mathfrak{c}_{2}(u)=u(n+1)$, then it is regular if and only if $\Psi_{\mathfrak{c}_{1}, \mathfrak{c}_{2}}^{a, b, c}(0) \neq 0$ or, equivalently, if and only if $\Phi_{\mathfrak{c}_{1}, \mathfrak{c}_{2}}^{a, b}(n+1) \neq 0$ and its resolvent kernel is given by

$$
R_{\mathfrak{c}_{1}, \mathfrak{c}_{2}}^{a, b, c}(k, s)=\frac{\rho_{\gamma}(s)}{a(0) \Psi_{\mathfrak{c}_{1}, \mathfrak{c}_{2}}^{a, b, c}(0)} \Phi_{\mathfrak{c}_{1}, \mathfrak{c}_{2}}^{a, b, c}(\min \{k, s\}) \Psi_{\mathfrak{c}_{1}, \mathfrak{c}_{2}}^{a, b, c}(\max \{k, s\}),
$$

for any $k, s=0, \ldots, n+1$.

Corollary 6 If the Dirichlet boundary value problem $\left(\mathcal{L}_{q}^{\gamma}, \mathfrak{c}_{1}, \mathfrak{c}_{2}\right)$ is interior, that is, $\mathfrak{c}_{1}(u)=u(1)$ and $\mathfrak{c}_{2}(u)=u(n)$, then it is regular if and only if $\Psi_{\mathfrak{c}_{1}, \mathfrak{c}_{2}}^{a, b, c}(1) \neq 0$ or, equivalently, if and only if $\Phi_{\mathfrak{c}_{1}, \mathfrak{c}_{2}}^{a, b}(n) \neq 0$ and its resolvent kernel is given by

$$
R_{\mathfrak{c}_{1}, \mathfrak{c}_{2}}^{a, b, c}(k, s)=\frac{\rho_{\gamma}(s)}{a(0) \Psi_{\mathfrak{c}_{1}, \mathfrak{c}_{2}}^{a, b, c}(1)} \Phi_{\mathfrak{c}_{1}, \mathfrak{c}_{2}}^{a, b, c}(\min \{k, s\}) \Psi_{\mathfrak{c}_{1}, \mathfrak{c}_{2}}^{a, b, c}(\max \{k, s\}),
$$

for any $k, s=0, \ldots, n+1$.

Corollary 7 If the Dirichlet boundary value problem $\left(\mathcal{L}_{q}^{\gamma}, \mathfrak{c}_{1}, \mathfrak{c}_{2}\right)$ is exterior-interior, that is, $\mathfrak{c}_{1}(u)=u(0)$ ans $\mathfrak{c}_{2}(u)=u(n)$, then it is regular if and only if $\Psi_{\mathfrak{c}_{1}, \mathfrak{c}_{2}}^{a, b, c}(0) \neq 0$ or, equivalently, if and only if $\Phi_{\mathfrak{c}_{1}, \mathfrak{c}_{2}}^{a, b, c}(n) \neq 0$ and its resolvent kernel is given by

$$
R_{\mathfrak{c}_{1}, \mathfrak{c}_{2}}^{a, b, c}(k, s)=\frac{\rho_{\gamma}(s)}{a(0) \Psi_{\mathfrak{c}_{1}, \mathfrak{c}_{2}}^{a, b, c}(0)} \Phi_{\mathfrak{c}_{1}, \mathfrak{c}_{2}}^{a, b, c}(\min \{k, s\}) \Psi_{\mathfrak{c}_{1}, \mathfrak{c}_{2}}^{a, b, c}(\max \{k, s\}),
$$

for any $k, s=0, \ldots, n+1$.

Corollary 8 If the Dirichlet boundary value problem $\left(\mathcal{L}_{q}^{\gamma}, \mathfrak{c}_{1}, \mathfrak{c}_{2}\right)$ is interior-exterior, that is, $\mathfrak{c}_{1}(u)=u(1)$ and $\mathfrak{c}_{2}(u)=u(n+1)$, then it is regular if and only if $\Psi_{\mathfrak{c}_{1}, \mathfrak{c}_{2}}^{a, b}(1) \neq 0$ or, equivalently, if and only if $\Phi_{\mathfrak{c}_{1}, \mathfrak{c}_{2}}^{a, b, c}(n+1) \neq 0$ and its resolvent kernel is given by

$$
R_{\mathfrak{c}_{1}, \mathfrak{c}_{2}}^{a, b, c}(k, s)=\frac{\rho_{\gamma}(s)}{a(0) \Psi_{\mathfrak{c}_{1}, \mathfrak{c}_{2}}^{a, b, c}(1)} \Phi_{\mathfrak{c}_{1}, \mathfrak{c}_{2}}^{a, b, c}(\min \{k, s\}) \Psi_{\mathfrak{c}_{1}, \mathfrak{c}_{2}}^{a, b, c}(\max \{k, s\}),
$$

for any $k, s=0, \ldots, n+1$.

Corollary 9 If the Dirichlet boundary value problem $\left(\mathcal{L}_{q}^{\gamma}, \mathfrak{c}_{1}, \mathfrak{c}_{2}\right)$ is exterior-Robin, that is, $\mathfrak{c}_{1}(u)=u(0), \mathfrak{c}_{2}(u)=\sigma_{23} u(n)+\sigma_{24} u(n+1)$ with $\sigma_{23} \sigma_{24} \neq 0$, then it is regular if and only if $\Psi_{\mathfrak{c}_{1}, \mathfrak{c}_{2}}^{a, b, c}(0) \neq 0$ or, equivalently, if and only if $\sigma_{23} \Phi_{\mathfrak{c}_{1}, \mathfrak{c}_{2}}^{a, b}(n) \neq$ $-\sigma_{24} \Phi_{\mathfrak{c}_{1}, \mathfrak{c}_{2}}^{a, b, c}(n+1)$ and its resolvent kernel is given by

$$
R_{\mathfrak{c}_{1}, \mathfrak{c}_{2}}^{a, b, c}(k, s)=\frac{\rho_{\gamma}(s)}{a(0) \Psi_{\mathfrak{c}_{1}, \mathfrak{c}_{2}}^{a, b, c}(0)} \Phi_{\mathfrak{c}_{1}, \mathfrak{c}_{2}}^{a, b, c}(\min \{k, s\}) \Psi_{\mathfrak{c}_{1}, \mathfrak{c}_{2}}^{a, b, c}(\max \{k, s\}),
$$

for any $k, s=0, \ldots, n+1$. 
Corollary 10 If the Dirichlet boundary value problem $\left(\mathcal{L}_{q}^{\gamma}, \mathfrak{c}_{1}, \mathfrak{c}_{2}\right)$ is interior-Robin, that is, $\mathfrak{c}_{1}(u)=u(1), \mathfrak{c}_{2}(u)=\sigma_{23} u(n)+\sigma_{24} u(n+1)$ with $\sigma_{23} \sigma_{24} \neq 0$, then it is regular if and only if $\Psi_{\mathfrak{c}_{1}, \mathfrak{c}_{2}}^{a, b}(1) \neq 0$ or, equivalently, if and only if $\sigma_{23} \Phi_{\mathfrak{c}_{1}, \mathfrak{c}_{2}}^{a, b, c}(n) \neq$ $-\sigma_{24} \Phi_{\mathfrak{c}_{1}, \mathfrak{c}_{2}}^{a, b}(n+1)$ and its resolvent kernel is given by

$$
R_{\mathfrak{c}_{1}, \mathfrak{c}_{2}}^{a, b, c}(k, s)=\frac{\rho_{\gamma}(s)}{a(0) \Psi_{\mathfrak{c}_{1}, \mathfrak{c}_{2}}^{a, b, c}(1)} \Phi_{\mathfrak{c}_{1}, \mathfrak{c}_{2}}^{a, b, c}(\min \{k, s\}) \Psi_{\mathfrak{c}_{1}, \mathfrak{c}_{2}}^{a, b, c}(\max \{k, s\})
$$

for any $k, s=0, \ldots, n+1$.

Corollary 11 If the Robin-Dirichlet boundary value problem $\left(\mathcal{L}_{q}^{\gamma}, \mathfrak{c}_{1}, \mathfrak{c}_{2}\right)$ is exterior, that is, $\mathfrak{c}_{1}(u)=\sigma_{11} u(0)+\sigma_{12} u(1)$ with $\sigma_{11} \sigma_{12} \neq 0, \mathfrak{c}_{2}(u)=u(n+1)$, then it is regular if and only if $\Phi_{\mathfrak{c}_{1}, \mathfrak{c}_{2}}^{a, b, c}(n+1) \neq 0$, or, equivalently, if and only if $\sigma_{11} \Psi_{\mathfrak{c}_{1}, \mathfrak{c}_{2}}^{a, b, c}(0) \neq$ $-\sigma_{12} \Psi_{\mathfrak{c}_{1}, \mathfrak{c}_{2}}^{a, b}(1)$ and its resolvent kernel is given by

$$
R_{\mathfrak{c}_{1}, \mathfrak{c}_{2}}^{a, b}(k, s)=-\frac{\rho_{\gamma}(s)}{a(n) \rho_{\gamma}(n) \Phi_{\mathfrak{c}_{1}, \mathfrak{c}_{2}}^{a, b, c}(n+1)} \Phi_{\mathfrak{c}_{1}, \mathfrak{c}_{2}}^{a, b, c}(\min \{k, s\}) \Psi_{\mathfrak{c}_{1}, \mathfrak{c}_{2}}^{a, b}(\max \{k, s\}),
$$

for any $k, s=0, \ldots, n+1$.

Corollary 12 If the Robin-Dirichlet boundary value problem $\left(\mathcal{L}_{q}^{\gamma}, \mathfrak{c}_{1}, \mathfrak{c}_{2}\right)$ is interior, that is, $\mathfrak{c}_{1}(u)=\sigma_{11} u(0)+\sigma_{12} u(1)$ with $\sigma_{11} \sigma_{12} \neq 0, \mathfrak{c}_{2}(u)=u(n)$, then it is regular if and only if $\Phi_{\mathfrak{c}_{1}, \mathfrak{c}_{2}}^{a, b, c}(n) \neq 0$ or, equivalently, if and only if $\sigma_{11} \Psi_{\mathfrak{c}_{1}, \mathfrak{c}_{2}}^{a, b}(0) \neq-\sigma_{12} \Psi_{\mathfrak{c}_{1}, \mathfrak{c}_{2}}^{a, b, c}(1)$ and its resolvent kernel is given by

$$
R_{\mathfrak{c}_{1}, \mathfrak{c}_{2}}^{a, b, c}(k, s)=-\frac{\rho_{\gamma}(s)}{a(n) \rho_{\gamma}(n) \Phi_{\mathfrak{c}_{1}, \mathfrak{c}_{2}}^{a, b, c}(n)} \Phi_{\mathfrak{c}_{1}, \mathfrak{c}_{2}}^{a, b, c}(\min \{k, s\}) \Psi_{\mathfrak{c}_{1}, \mathfrak{c}_{2}}^{a, b, c}(\max \{k, s\}),
$$

for any $k, s=0, \ldots, n+1$.

\subsection{Periodic problems}

For periodic boundary value problems, the conditions that are needed for regularity cannot be simplified, since the functions of the fundamental basis involve values at both ends. Therefore, we assume in both cases that the problem is regular and, under this hypothesis, we will determine the resolving kernels.

Let us consider the periodic boundary conditions pair $\left(\mathfrak{c}_{1}, \mathfrak{c}_{2}\right)$ of first kind, that is, it is determined by the matrix $\left[\begin{array}{cccc}\sigma_{11} & \sigma_{12} & \sigma_{13} & \sigma_{14} \\ \sigma_{21} & 0 & 0 & \sigma_{24}\end{array}\right]$, where $\sigma_{12} \cdot \sigma_{13} \cdot \sigma_{21} \cdot \sigma_{24} \neq 0$.

Then,

$$
\begin{aligned}
& g_{11}=\sigma_{11} \phi_{\mathbf{c}_{1}, \mathfrak{c}_{2}}^{a, b}(0)+\sigma_{12} \phi_{\mathfrak{c}_{1}, \mathfrak{c}_{2}}^{a, b, c}(1), g_{12}=\sigma_{13} \psi_{\mathfrak{c}_{1}, \mathfrak{c}_{2}}^{a, b}(n)+\sigma_{14} \psi_{\mathfrak{c}_{1}, \mathfrak{c}_{2}}^{a, b, c}(n+1), \\
& g_{21}=\sigma_{21} \phi_{\mathfrak{c}_{1}, \mathfrak{c}_{2}}^{a, b}(0), \quad g_{22}=\sigma_{24} \psi_{\mathfrak{c}_{1}, \mathfrak{c}_{2}}^{a, b, c}(n+1) .
\end{aligned}
$$


Proposition 12 If the periodic boundary value problem $\left(\mathcal{L}_{q}^{\gamma}, \mathfrak{c}_{1}, \mathfrak{c}_{2}\right)$ of first kind is regular, its resolvent kernel is given by

$$
\begin{aligned}
& R_{\mathfrak{c}_{1}, \mathfrak{c}_{2}}^{a, b, c}(k, s)=-\frac{\rho_{\gamma}(s) \phi_{\mathfrak{c}_{1}, \mathfrak{c}_{2}}^{a, b}(\min \{k, s\}) \psi_{\mathfrak{c}_{1}}^{a, \mathfrak{c}_{2}}(\max \{k, s\})}{a(0) w\left[\phi_{\mathfrak{c}_{1}, \mathfrak{c}_{2}}^{a, b, c}, \psi_{\mathfrak{c}_{1}, \mathfrak{c}_{2}}^{a, b, c}\right](0)} \\
& -\frac{\rho_{\gamma}(s)\left[g_{11} \psi_{\mathfrak{c}_{1}, \mathfrak{c}_{2}}^{a, b, c}(k) \psi_{\mathfrak{c}_{1}, \mathfrak{c}_{2}}^{a, b, c}(s)-g_{22} \phi_{\mathfrak{c}_{1}, \mathfrak{c}_{2}}^{a, b, c}(k) \phi_{\mathfrak{c}_{1}, \mathfrak{c}_{2}}^{a, b, c}(s)\right]}{w\left[\phi_{\mathfrak{c}_{1}, \mathfrak{c}_{2}}^{a, b, c}, \psi_{\mathfrak{c}_{1}, \mathfrak{c}_{2}}^{a, b, c}\right](0) w\left[\phi_{\mathfrak{c}_{1}, \mathfrak{c}_{2}}^{a, b, c}, \psi_{\mathfrak{c}_{1}, \mathfrak{c}_{2}}^{a, b, c}\right](n)} \\
& -\frac{\rho_{\gamma}(s)\left[g_{12} \psi_{\mathfrak{c}_{1}, \mathfrak{c}_{2}}^{a, b, c}(k) \phi_{\mathfrak{c}_{1}, \mathfrak{c}_{2}}^{a, b}(s)-g_{21} \phi_{\mathfrak{c}_{1}, \mathfrak{c}_{2}}^{a, b, c}(k) \psi_{\mathfrak{c}_{1}, \mathfrak{c}_{2}}^{a, b, c}(s)\right]}{w\left[\phi_{\mathfrak{c}_{1}, \mathfrak{c}_{2}}^{a, b}, \psi_{\mathfrak{c}_{1}, \mathfrak{c}_{2}}^{a, b, c}\right](0) w\left[\phi_{\mathfrak{c}_{1}, \mathfrak{c}_{2}}^{a, b, c}, \psi_{\mathfrak{c}_{1}, \mathfrak{c}_{2}}^{a, b, c}\right](n)} \\
& -\frac{\left(a(0)+\sigma_{12}\right) \psi_{\mathfrak{c}_{1}, \mathfrak{c}_{2}}^{a, b, c}(k)}{w\left[\phi_{\mathfrak{c}_{1}, \mathfrak{c}_{2}}^{a, b,}, \psi_{\mathfrak{c}_{1}, \mathfrak{c}_{2}}^{a, b, c}\right](n)} \varepsilon_{s}(0) \\
& -\frac{\rho_{\gamma}(n+1)\left[\sigma_{13} \psi_{\mathfrak{c}_{1}, \mathfrak{c}_{2}}^{a, b, c}(k)-c(n) \phi_{\mathfrak{c}_{1}, \mathfrak{c}_{2}}^{a, b}(k)\right]}{w\left[\phi_{\mathfrak{c}_{1}, \mathfrak{c}_{2}}^{a, b}, \psi_{\mathfrak{c}_{1}, \mathfrak{c}_{2}}^{a, b, c}\right](0)} \varepsilon_{s}(n+1),
\end{aligned}
$$

for any $k, s=0, \ldots, n+1$.

Let us consider the periodic boundary conditions pair $\left(\mathfrak{c}_{1}, \mathfrak{c}_{2}\right)$ of second kind, that is, it is determined by the matrix $\left[\begin{array}{cccc}\sigma_{11} & -a(0) & 0 & \sigma_{14} \\ \sigma_{21} & 0 & -c(n) & \sigma_{24}\end{array}\right]$, where $\sigma_{21} \cdot \sigma_{14} \neq 0$. Then,

$$
\begin{aligned}
& g_{11}=\sigma_{11} \phi_{\mathfrak{c}_{1}, \mathfrak{c}_{2}}^{a, b, c}(0)-a(0) \phi_{\mathfrak{c}_{1}, \mathfrak{c}_{2}}^{a, b, c}(1), g_{12}=\sigma_{14} \psi_{\mathfrak{c}_{1}, \mathfrak{c}_{2}}^{a, b, c}(n+1), \\
& g_{21}=\sigma_{21} \phi_{\mathfrak{c}_{1}, \mathfrak{c}_{2}}^{a, b, c}(0), \quad g_{22}=-c(n) \psi_{\mathfrak{c}_{1}, \mathfrak{c}_{2}}^{a, b, c}(n)+\sigma_{24} \psi_{\mathfrak{c}_{1}, \mathfrak{c}_{2}}^{a, b, c}(n+1) .
\end{aligned}
$$

Proposition 13 If the periodic boundary value problem $\left(\mathcal{L}_{q}^{\gamma}, \mathfrak{c}_{1}, \mathfrak{c}_{2}\right)$ of second kind is regular, its resolvent kernel is given by

$$
\begin{aligned}
R_{\mathfrak{c}_{1}, \mathfrak{c}_{2}}^{a, b, c}(k, s)= & -\frac{\rho_{\gamma}(s) \phi_{\mathfrak{c}_{1}, \mathfrak{c}_{2}}^{a, b, c}(\min \{k, s\}) \psi_{\mathfrak{c}_{1}, \mathfrak{c}_{2}}^{a, b}(\max \{k, s\})}{a(0) w\left[\phi_{\mathfrak{c}_{1}, \mathfrak{c}_{2}}^{a, b}, \psi_{\mathfrak{c}_{1}}^{a, \mathfrak{c}_{2}}\right](0)} \\
& -\frac{\rho_{\gamma}(s)\left[g_{11} \psi_{\mathfrak{c}_{1}, \mathfrak{c}_{2}}^{a, b}(k) \psi_{\mathfrak{c}_{1}, \mathfrak{c}_{2}}^{a, b, c}(s)-g_{22} \phi_{\mathfrak{c}_{1}, \mathfrak{c}_{2}}^{a, b, c}(k) \phi_{\mathfrak{c}_{1}, \mathfrak{c}_{2}}^{a, b}(s)\right]}{w\left[\phi_{\mathfrak{c}_{1}, \mathfrak{c}_{2}}^{a, b}, \psi_{\mathfrak{c}_{1}, \mathfrak{c}_{2}}^{a, c}\right](0) w\left[\phi_{\mathbf{c}_{1}, \mathfrak{c}_{2}}^{a, b}, \psi_{\mathfrak{c}_{1}, \mathfrak{c}_{2}}^{a, b}\right](n)} \\
& -\frac{\rho_{\gamma}(s)\left[g_{12} \psi_{\mathfrak{c}_{1}, \mathfrak{c}_{2}}^{a, b}(k) \phi_{\mathfrak{c}_{1}, \mathfrak{c}_{2}}^{a, b}(s)-g_{21} \phi_{\mathbf{c}_{1}, \mathfrak{c}_{2}}^{a, b}(k) \psi_{\mathfrak{c}_{1}, \mathfrak{c}_{2}}^{a, b}(s)\right]}{w\left[\phi_{\mathfrak{c}_{1}, \mathfrak{c}_{2}}^{a, b, c}, \psi_{\mathfrak{c}_{1}, \mathfrak{c}_{2}}^{a, b, c}\right](0) w\left[\phi_{\mathfrak{c}_{1}, \mathfrak{c}_{2}}^{a, b, c}, \psi_{\mathfrak{c}_{1}, \mathfrak{c}_{2}}^{a, b}\right](n)},
\end{aligned}
$$

for any $k, s=0, \ldots, n+1$.

\section{The inverse of a generalized Jacobi matrix}

The regularity conditions of the boundary value problem (9) have been described in previous section for each kind of conditions pair $\left(\mathfrak{c}_{1}, \mathfrak{c}_{2}\right)$, and coincide with the 
invertibility conditions of the generalized Jacobi matrix of order $n+2$

$$
\mathbf{M}=\left[\begin{array}{cccccccc}
\sigma_{11} & \sigma_{12} & 0 & 0 & \cdots & 0 & \sigma_{13} & \sigma_{14} \\
-c_{0} & b_{1} & -a_{1} & 0 & \cdots & 0 & 0 & 0 \\
0 & -c_{1} & b_{2} & -a_{2} & \cdots & 0 & 0 & 0 \\
\vdots & \vdots & \vdots & \vdots & \vdots & \vdots & \vdots & \vdots \\
0 & 0 & 0 & 0 & \cdots & -c_{n-1} & b_{n} & -a_{n} \\
\sigma_{21} & \sigma_{22} & 0 & 0 & \cdots & 0 & \sigma_{23} & \sigma_{24}
\end{array}\right]
$$

Moreover, determining its inverse, $\mathrm{M}^{-1}=\mathrm{R}=\left(r_{k s}\right)$, is equivalent to obtain the inverse of the linear operator $\mathcal{L}_{q}^{\gamma}$ on $(9)$, that, according to Definition 10 and Proposition 5 is

$$
\left(\mathcal{L}_{q}^{\gamma}\right)^{-1}(f)(k)=\sum_{s \in \mathbf{I}} R(k, s) f(s)
$$

So the entries $r_{k s}$ of matrix $\mathrm{R}$ correspond to the values of the resolvent kernel $R(k, s), k, s=0, \ldots, n+1$. The general expression for this kernel is described on Corollary 3, and particular expressions of $R(k, s)$ for fixed conditions appear all along Section 6, but all them in terms of the Schrödinger equation's solutions $\phi_{\mathfrak{c}_{1}, \mathfrak{c}_{2}}^{a, b}$ and $\psi_{\mathfrak{c}_{1}, \mathfrak{c}_{2}}^{a, b}$, so they are not yet explicit expressions. Therefore, to obtain the explicit values of the entries of $\mathrm{R}$ we just need to compute explicitly the functions $\phi_{\mathfrak{c}_{1}, \mathfrak{c}_{2}}^{a, b, c}$ and $\psi_{\mathfrak{c}_{1}, \mathfrak{c}_{2}}^{a, b, c}$. To compute these solutions we will use recent advances in the study of difference equations developed by the authors in [9], a work devoted to the study of general second order difference equations as the one associated with the Schrödinger equation and described in (3). In particular, in Section 7 of this paper it has been proved that the solution of that kind of equations can be expressed as a linear combination of the functions $P_{k}(x, y)$ called $k$-th Chebyshev functions and defined for any $x, y \in \mathcal{C}(\mathbb{Z})$ as

$$
P_{0}(x, y)=1, \quad P_{-1}(x, y)=0 \text { and } P_{k}(x, y)=\sum_{m=0}^{\left\lfloor\frac{k}{2}\right\rfloor}(-1)^{m} \sum_{\alpha \in \ell_{k}^{m}} x^{\bar{\alpha}} y^{\alpha}, k \geq 1 .
$$

We reproduce here some brief explanations about the notation involved in (12), for the sake of completeness. The parameter $\alpha=\left(\alpha_{1}, \ldots, \alpha_{p}\right)$ is a binary multi-index of order $p$; i.e. $\alpha$ is a $p$-tuple $\alpha=\left(\alpha_{1}, \ldots, \alpha_{p}\right) \in\{0,1\}^{p}$, and its length is defined as $|\alpha|=\sum_{j=1}^{p} \alpha_{j} \leq p$. Given $\alpha \in\{0,1\}^{p}$ and a function $a \in \mathcal{C}(\mathbb{Z})$, we consider the value $a^{\alpha}=\prod_{j=1}^{p} a(j)^{\alpha_{j}}$. Given a positive integer $p$, we denote by $i_{1}, \ldots, i_{m}$ the indices such that $1 \leq i_{1}<\cdots<i_{m} \leq p$ and $\alpha_{i_{j}}=1, j=1, \ldots, m$. We just need to consider the binary multi-indexes $\alpha$ of order $p$ in the set $\ell_{p}$ defined as

(i) $\ell_{p}^{0}=\{\alpha:|\alpha|=0\}=\{(0, \ldots, 0)\}$, for a positive integer $p$,

(ii) $\ell_{p}^{1}=\left\{\alpha: \alpha_{p}=0\right.$ and $\left.|\alpha|=1\right\}$, for $p \geq 2$,

(iii) $\ell_{p}^{m}=\left\{\alpha: \alpha_{p}=0,|\alpha|=m\right.$ and $\left.i_{j+1}-i_{j} \geq 2, j=1, \ldots, m-1\right\}$, for $p \geq 4$ and $m=2, \ldots,\left\lfloor\frac{p}{2}\right\rfloor$. 
Finally, $\bar{\alpha}$ is the binary multi-index of the same order as $\alpha$ defined by

$$
\bar{\alpha}_{i_{j}}=\bar{\alpha}_{i_{j}+1}=0, j=1, \ldots, m, \text { and } \bar{\alpha}_{i}=1 \text { otherwise. }
$$

The name of Chebyshev function for (12) is justified due to its relation with the usual Chebyshev polynomials of second kind, since $P_{k}(x, y)$ can be identified with them when $x$ and $y$ are constant sequences. In that case, $P_{0}(x, y)=1$ and $P_{-1}(x, y)=0$ and moreover, since $\# \ell_{k}^{m}=\left(\begin{array}{c}k-m \\ m\end{array}\right)$ for any positive integer $k$, we obtain that

$$
P_{k}(x, y)=\sum_{m=0}^{\left\lfloor\frac{k}{2}\right\rfloor}(-1)^{m}\left(\begin{array}{c}
k-m \\
m
\end{array}\right) x^{k-2 m} y^{m} .
$$

Clearly, for any $k \geq-1$ and any constant sequence $x$, we have

$$
U_{k}(x)=P_{k}(2 x, 1)=\sum_{m=0}^{\left\lfloor\frac{k}{2}\right\rfloor}(-1)^{m}\left(\begin{array}{c}
k-m \\
m
\end{array}\right)(2 x)^{k-2 m},
$$

that is known as the standard $k$-th Chebyshev polynomial of second kind, see [2] and also $[4,8]$. Definitely, for constant sequences $x$ and $y$, it is satisfied

$$
P_{k}(x, y)=y^{\frac{k}{2}} \sum_{m=0}^{\left\lfloor\frac{k}{2}\right\rfloor}(-1)^{m}\left(\begin{array}{c}
k-m \\
m
\end{array}\right)\left(\frac{x}{\sqrt{y}}\right)^{k-2 m}=y^{\frac{k}{2}} U_{k}\left(\frac{x}{2 \sqrt{y}}\right), \quad k \geq 1 .
$$

Hence, we are able to compute the basis of solutions $\left\{\phi_{\mathfrak{c}_{1}, \mathfrak{c}_{2}}^{a, b, c}(k), \psi_{\mathfrak{c}_{1}, \mathfrak{c}_{2}}^{a, b, c}(k)\right\}$ of the homogeneous Schrödinger equation $\mathcal{L}_{q}^{\gamma}(u)=0$ on $\stackrel{\circ}{\mathbf{I}}$ applying the results showed in [9] on second order difference equations, that is through a linear combination of the Chebyshev functions $P_{k}(b, a c)$ and $P_{k}\left(b_{m}, a_{m} c_{m}\right)$, where $a, b, c \in \mathcal{C}(\mathbf{I})$ are the coefficients of the second order difference equation associated to the Schrödinger equation, and given $a \in \mathcal{C}(\mathbb{Z})$ and $m \in \mathbb{N}$, the function $a_{m}$ corresponds to the $m$-shift of $a$, so $a_{m}=a(k+m)$. To obtain the final expression of $R(k, s)$, and so, of the entries of the inverse matrix $R$, we only need to impose the corresponding conditions according to the classification of the boundary value problems made in Section 3 .

This strategy has already been applied successfully for the inversion of Jacobi matrices, that is, when $\sigma_{13}=\sigma_{14}=\sigma_{21}=\sigma_{22}=0$, see [11], and also to obtain the explicit inverse of the so-called tridiagonal $(p, r)$-Toeplitz matrix [10], which are those Jacobi matrices with $\sigma_{11}=b(0), \sigma_{12}=-a(0), \sigma_{23}=-c(n)$ and $\sigma_{24}=$ $b(n+1)$, in which each diagonal is a quasi-periodic sequence, that is $a(p+j)=r a(j)$, $b(p+j)=r b(j)$ and $c(p+j)=r c(j)$, where $p \in \mathbb{N}$ and $r \in \mathbb{R}$. The interested reader can find in [3] a more specific and direct approach for the inversion of this kind of matrices. In addition, the same technique has been used to invert some circulant matrices that can be related to second order difference equations with constant coefficients, see [6].

To illustrate the same strategy for a generalized Jacobi matrix, we consider the following matrix $\mathrm{M}$ of order 7 as an example, and we will verify that its inverse 
corresponds to the matrix R.

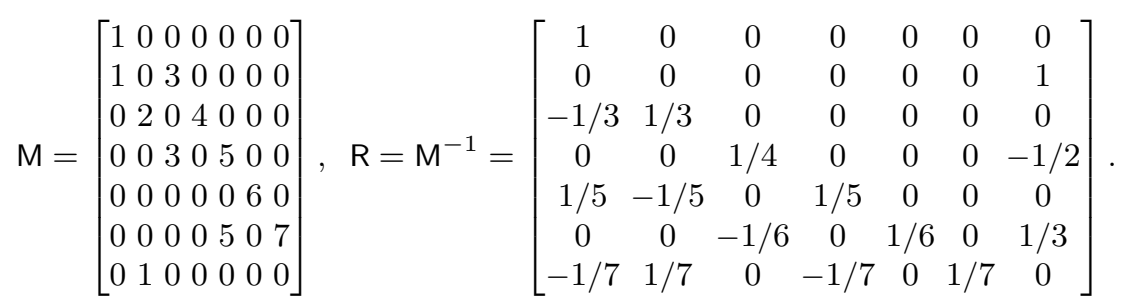

For matrix $\mathrm{M}, \sigma_{11}=\sigma_{22}=1$ and $\sigma_{12}=\sigma_{13}=\sigma_{14}=\sigma_{21}=\sigma_{23}=\sigma_{24}=0$, and then $\mathrm{C}_{34}=0$. Therefore, according to the classification we made in Section 3 , the pair $\left(\mathfrak{c}_{1}, \mathfrak{c}_{2}\right)$ corresponds to initial unilateral boundary conditions, so we have to use the resolvent kernel for unilateral problems obtained in Proposition 7 to compute $\mathrm{R}=\mathrm{M}^{-1}$. To do this, we first need to obtain the basis of solutions $\left\{\phi_{\mathfrak{c}_{1}, \mathfrak{c}_{2}}^{a, b}(k), \psi_{\mathfrak{c}_{1}, \mathfrak{c}_{2}}^{a, b, c}(k)\right\}$ of the homogeneous Schrödinger equation $\mathcal{L}_{q}^{\gamma}(u)=0$ on $\stackrel{\circ}{\mathbf{I}}=\{1, \ldots, 5\}$, which for matrix $\mathrm{M}$ is the uncoupled equation

$$
(k+2) u(k+1)+k u(k-1)=0, \quad k=1, \ldots, 5,
$$

where $a(k)=-k-2, b(k)=0$ and $c(k)=-k-1$.

Applying the result shown in [9, Theorem 7.4] to obtain the Green function for a second order difference equation through a linear combination of the Chebyshev functions $P_{k}(b, a c)$ and $P_{k}\left(b_{1}, a_{1} c_{1}\right)$, the Green function for the above uncoupled equation for any $k, s=0, \ldots, 6$ is

$$
\begin{aligned}
& g(2 k+1,2 s)=\frac{(-1)^{|k-s|}}{2 s+2}\left[\frac{\min \{k, s\}+1}{\max \{k, s\}+1}\right]^{\operatorname{sign}(k-s)}, \\
& g(2 k, 2 s+1)=\frac{(-1)^{|k-s|-1}}{2 k+1},
\end{aligned}
$$

and null otherwise. Hence, the unique solution of the initial value problem

$$
(k+2) u(k+1)+k u(k-1)=0 \text { on } \stackrel{\circ}{\mathbf{I}} \text { and } u(0)=\alpha, \quad u(1)=\beta,
$$

is determined by

$$
u(2 k)=\frac{(-1)^{k}}{2 k+1} \alpha, \quad k=0,1,2,3, \quad \text { and } \quad u(2 k+1)=\frac{(-1)^{k}}{k+1} \beta, \quad k=0,1,2 ;
$$

see [9, Theorem 4.3].

Since for initial unilateral problems $\phi_{\mathbf{c}_{1}, \mathfrak{c}_{2}}^{a, b, c}(0)=-a(n) \rho_{\gamma}(n) \sigma_{12}$ and $\phi_{\mathbf{c}_{1}, \mathfrak{c}_{2}}^{a, b, c}(1)=$ $a(n) \rho_{\gamma}(n) \sigma_{11}$, whereas that $\psi_{\mathfrak{c}_{1}, \mathfrak{c}_{2}}^{a, b, c}(0)=-a(n) \rho_{\gamma}(n) \sigma_{22}$ and $\psi_{\mathfrak{c}_{1}, \mathfrak{c}_{2}}^{a, b}(1)=a(n) \rho_{\gamma}(n) \sigma_{21}$, for this example

$$
\phi_{\mathfrak{c}_{1}, \mathfrak{c}_{2}}^{a, b, c}(0)=\psi_{\mathfrak{c}_{1}, \mathfrak{c}_{2}}^{a, b, c}(1)=0, \quad \psi_{\mathfrak{c}_{1}, \mathfrak{c}_{2}}^{a, b, c}(0)=-\phi_{\mathfrak{c}_{1}, \mathfrak{c}_{2}}^{a, b, c}(1)=-a(5) \rho_{\gamma}(5)=7 \cdot 6=42 .
$$

The expressions of the solution $u$ for these initial conditions finally determine the basis $\left\{\phi_{\mathfrak{c}_{1}, \mathfrak{c}_{2}}^{a, b}, \psi_{\mathfrak{c}_{1}, \mathfrak{c}_{2}}^{a, b, c}\right\}$,

$$
\begin{aligned}
& \phi_{\mathfrak{c}_{1}, \mathfrak{c}_{2}}^{a, b, c}(2 k)=0, \quad k=0,1,2,3, \quad \text { and } \quad \phi_{\mathfrak{c}_{1}, \mathfrak{c}_{2}}^{a, b, c}(2 k+1)=(-1)^{k+1} \frac{42}{k+1}, \quad k=0,1,2, \\
& \psi_{\mathfrak{c}_{1}, \mathfrak{c}_{2}}^{a, b, c}(2 k)=(-1)^{k} \frac{42}{2 k+1}, \quad k=0,1,2,3, \quad \text { and } \quad \psi_{\mathfrak{c}_{1}, \mathfrak{c}_{2}}^{a, b, c}(2 k+1)=0, \quad k=0,1,2 .
\end{aligned}
$$


So, we are now ready to compute the resolvent kernel associated to our initial unilateral problem, given for any $k, s=0, \ldots, 6$ by

$$
\begin{aligned}
R_{\mathfrak{c}_{1}, \mathfrak{c}_{2}}^{a, b, c}(k, s) & =\frac{\rho_{\gamma}(s)\left[\phi_{\mathfrak{c}_{1}, \mathfrak{c}_{2}}^{a, b}(k) \psi_{\mathfrak{c}_{1}, \mathfrak{c}_{2}}^{a, b, c}(s)-\phi_{\mathfrak{c}_{1}, \mathfrak{c}_{2}}^{a, b}(\min \{k, s\}) \psi_{\mathfrak{c}_{1}, \mathfrak{c}_{2}}^{a, b, c}(\max \{k, s\})\right]}{a(0) w\left[\phi_{\mathfrak{c}_{1}, \mathfrak{c}_{2}}^{a, b}, \psi_{\mathfrak{c}_{1}, \mathfrak{c}_{2}}^{a, c}\right](0)} \\
& +\frac{\left[\phi_{\mathfrak{c}_{1}, \mathfrak{c}_{2}}^{a, b, c}(k)-a(0) \psi_{\mathfrak{c}_{1}, \mathfrak{c}_{2}}^{a, b}(k)\right]}{w\left[\phi_{\mathfrak{c}_{1}, \mathfrak{c}_{2}}^{a, b}, \psi_{\mathfrak{c}_{1}, \mathfrak{c}_{2}}^{a, c}\right](5)} \varepsilon_{s}(0)+\frac{\rho_{\gamma}(6) c(6) \phi_{\mathfrak{c}_{1}, \mathfrak{c}_{2}}^{a, b}(k)}{w\left[\phi_{\mathbf{c}_{1}, \mathfrak{c}_{2}}^{a, b}, \psi_{\mathfrak{c}_{1}, \mathfrak{c}_{2}}^{a, c}\right](0)} \varepsilon_{s}(6),
\end{aligned}
$$

where $\rho_{\gamma}(k)=\prod_{s=0}^{k-1} \frac{a(s)}{c(s)}, a(0)=-2, c(6)=-7, w\left[\phi_{\mathbf{c}_{1}, \mathfrak{c}_{2}}^{a, b, c}, \psi_{\mathbf{c}_{1}, \mathfrak{c}_{2}}^{a, b, c}\right](0)=1764$ and $w\left[\phi_{\mathfrak{c}_{1}, \mathfrak{c}_{2}}^{a, b, c}, \psi_{\mathfrak{c}_{1}, \mathfrak{c}_{2}}^{a, b, c}\right](5)=84$.

Simply by replacing then the values of $\phi_{\mathfrak{c}_{1}, \mathfrak{c}_{2}}^{a, b, c}$ and $\psi_{\mathfrak{c}_{1}, \mathfrak{c}_{2}}^{a, b}$, we obtain

$$
\begin{aligned}
& R_{\mathfrak{c}_{1}, \mathfrak{c}_{2}}^{a, b}(0,0)=R_{\mathfrak{c}_{1}, \mathfrak{c}_{2}}^{a, b, c}(1,6)=1, \quad R_{\mathfrak{c}_{1}, \mathfrak{c}_{2}}^{a, b, c}(2,0)=-R_{\mathfrak{c}_{1}, \mathfrak{c}_{2}}^{a, b, c}(2,1)=-R_{\mathfrak{c}_{1}, \mathfrak{c}_{2}}^{a, b, c}(5,6)=-1 / 3, \\
& R_{\mathfrak{c}_{1}, \mathfrak{c}_{2}}^{a, b, c}(3,2)=1 / 4, \quad R_{\mathfrak{c}_{1}, \mathfrak{c}_{2}}^{a, b, c}(3,6)=-1 / 2, \\
& R_{\mathfrak{c}_{1}, \mathfrak{c}_{2}}^{a, b, c}(4,0)=-R_{\mathfrak{c}_{1}, \mathfrak{c}_{2}}^{a, b}(4,1)=R_{\mathfrak{c}_{1}, \mathfrak{c}_{2}}^{a, b}(4,3)=1 / 5, \\
& R_{\mathfrak{c}_{1}, \mathfrak{c}_{2}}^{a, b, c}(5,2)=-R_{\mathfrak{c}_{1}, \mathfrak{c}_{2}}^{a, b}(5,4)=-1 / 6, \\
& R_{\mathfrak{c}_{1}, \mathfrak{c}_{2}}^{a, b, c}(6,0)=-R_{\mathfrak{c}_{1}, \mathfrak{c}_{2}}^{a, b, c}(6,1)=R_{\mathfrak{c}_{1}, \mathfrak{c}_{2}}^{a, c}(6,3)=-R_{\mathfrak{c}_{1}, \mathfrak{c}_{2}}^{a, b, c}(6,5)=-1 / 7
\end{aligned}
$$

and all other values of $R_{\mathfrak{c}_{1}, \mathfrak{c}_{2}}^{a, b}(k, s), k, s=0, \ldots, 6$ are null. Therefore, these values of the resolvent kernel $R(k, s)$ correspond to the entries $r_{k s}$ of matrix $\mathrm{R}$, as we had announced.

Acknowledgements This work has been partly supported by the Spanish Program I+D+i (Ministerio de Economía y Competitividad) under projects MTM2017-85996-R and MTM201790682-REDT.

\section{References}

1. R.P. Agarwal. Difference equations and inequalities. Theory, methods, and applications. Monographs and Textbooks in Pure and Applied Mathematics, 228. Marcel Dekker, Inc., New York, 2000

2. D. Aharanov, A. Beardon and K. Driver. Fibonacci, Chebyshev and orthogonal polynomials. Am. Math. Mon., 112:612-630, 2005.

3. M. Andelić, C.M. da Fonseca. Some comments on tridiagonal $(p, r)$-Toeplitz matrices. Linear Algebra Appl., 572:46-50, 2019.

4. E. Bendito, A. Carmona and A.M. Encinas. Eigenvalues, Eigenfunctions and Green's Functions on a Path via Chebyshev Polynomials. Appl. Anal. Discr. Math., 3:282-302, 2009.

5. A. Carmona, A.M. Encinas and M. Mitjana. Green matrices associated with generalized linear polyominoes. Linear Algebra Appl., 468:38-47, 2015.

6. A. Carmona, A.M. Encinas, S. Gago, M.J. Jiménez and M. Mitjana. The inverses of some circulant matrices. Appl. Math. Comput., 270:785-793, 2015.

7. E.A. Coddington and N. Levinson. Theory of Ordinary Differential Equations. McGraw Hill, 1995.

8. A. M. Encinas and M.J. Jiménez. Floquet Theory for second order linear difference equations. J. Differ. Equ. Appl., 22:353-375, 2016.

9. A. M. Encinas and M.J. Jiménez. Second order linear difference equations. J. Diff. Equ. Appl., 24(3):305-343, 2018. 
10. A. M. Encinas and M.J. Jiménez. Explicit inverse of a tridiagonal (p,r)-Toeplitz matrix. Linear Algebra Appl., 542:402-421, 2018.

11. A. M. Encinas and M.J. Jiménez. Explicit inverse of nonsingular Jacobi matrices. Discrete Appl. Math., https://doi.org/10.1016/j.dam.2019.03.005, 2019.

12. M. A. El-Shehawey, Gh. A. El-Shreef and A. Sh. Al-Henawy. Analytical inversion of general periodic tridiagonal matrices. J. Math. Anal. Appl., 345:123-134, 2008.

13. K. Filipiak, A. Markiewicz, A. Sawikowska. Determinants of multidiagonal matrices. Electron. J. Linear Algebra, 25:102-118, 2012.

14. C. M. da Fonseca and J. Petronilho. Explicit inverse of some tridiagonal matrices. Linear Algebra Appl., 325:7-21, 2001.

15. C. M. da Fonseca and J. Petronilho. Explicit inverse of a tridiagonal $k$-Toeplitz matrix. Numer. Math., 100:457-482, 2005.

16. F. P. Gantmacher and M. G. Krein. Oscillation matrices and kernels and small vibrations of mechanical systems. AMS Chelsea Publishing, Providence, RI, 2002 (Translation based on the 1941 Russian original).

17. J.T. Jia, Y.L. Jiang. Symbolic algorithm for solving cyclic penta-diagonal linear systems. Numer. Algor., 63:357-367, 2013.

18. J. Jia, Q. Kong, T. Sogabe. A new algorithm for solving nearly penta-diagonal Toeplitz linear systems. Comput. Math. Appl. 63:1238-1243, 2012.

19. R. K. Mallik. The inverse of a tridiagonal matrix. Linear Algebra Appl., 325:109-139, 2001.

20. J. C. Mason and D. C. Handscomb, Chebyshev Polynomials. Chapman \& Hall/CRC, 2003.

21. J. J. McDonald, R. Nabben, M. Neumann, H. Schneider and M. J. Tsatsomeros. Inverse tridiagonal Z-Matrices. Linear Multilinear Algebra, 45:75-97, 1998.

22. G. Meurant. A review on the inverse of symmetric tridiagonal and block tridiagonal matrices. SIAM J. Matrix Anal. Appl., 13:707-728, 1992.

23. S.N. Neossi Nguetchue, S. Abelman. A computational algorithm for solving nearly pentadiagonal linear systems. Appl. Math. Comput. 203:629-634, 2008.

24. R. A. Usmani. Inversion of Jacobi's tridiagonal matrix. Comput. Math. Appl., 27:59-66, 1994.

25. R. A. Usmani. Inversion of a tridiagonal Jacobi matrix. Linear Algebra Appl., 212/213:413414, 1994. 